\title{
Suzuki-Miyaura Cross-Coupling of 1,1-Dichloro-1-alkenes with 9-Alkyl-9-BBN.
}

Frédéric Liron, Céline Fosse, Alban Pernolet and Emmanuel Roulland*

Institut de Chimie des Substances Naturelles, CNRS,

Avenue de la Terrasse, 91198 Gif-sur-Yvette, France

\section{emmanuel.roulland@icsn.cnrs-gif.fr}

\section{Supporting Information}

Table of Contents:

General and Material. S3

Experimental procedures and characterrization data:

Typical procedure for the preparation of 1,1-dichloro alkenes $(\operatorname{method~A):............S3~}$

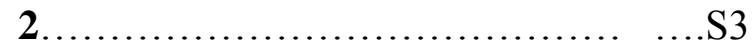
4. S3

Typical procedure for the preparation of 1,1-dichloro alkenes $(\operatorname{method} B)$ :.......S4 1................................... S4
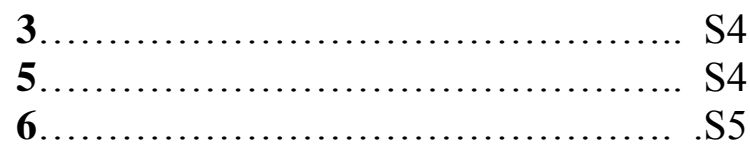

Typical procedure for stereoselective mono coupling:............................. S5

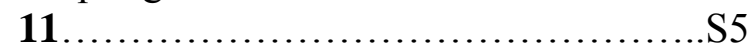

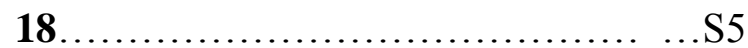

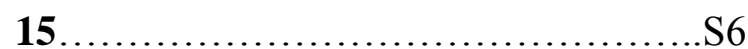

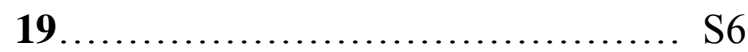

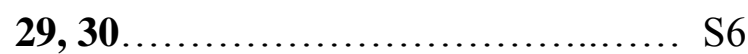

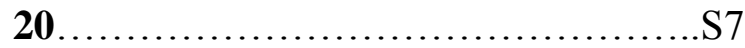

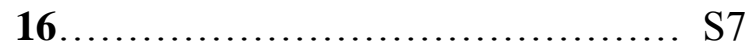

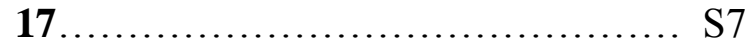

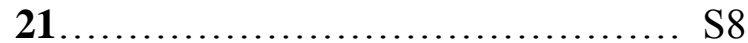

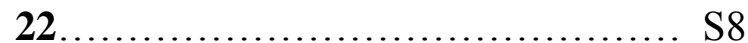

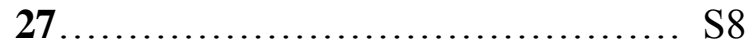

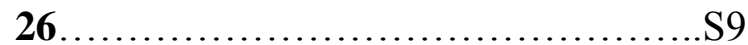

25-cis, 25-trans......................... S9

23-cis, 23-trans...................... S 10

24-cis+trans......................... 10
Typical procedure for non-selective crosscoupling of 1,1-dichloro alkenes:....... S10

20, 28.............................. S1 1

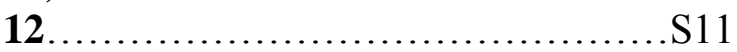

Typical procedure for second coupling, leading to tri-substituted olefins:........ S12

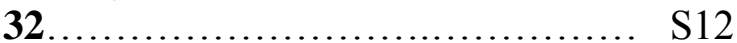

$31 \ldots \ldots \ldots \ldots \ldots \ldots \ldots \ldots \ldots \ldots \ldots \ldots \ldots \ldots \ldots \ldots, \quad \mathrm{S} 12$

33-Z ............................ S12

33-E............................. S13

Typical procedure for Suzuki crosscoupling with phenylboronic acid:..... S13

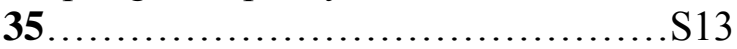

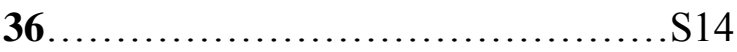

${ }^{1} \mathrm{H}$ and ${ }^{13} \mathrm{C}$ NMR:

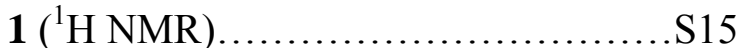

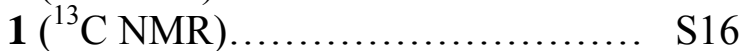

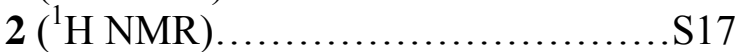

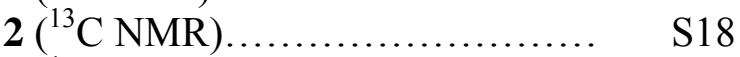

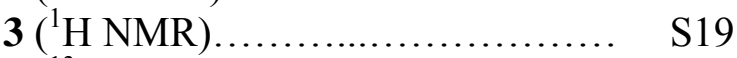

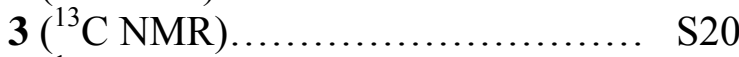

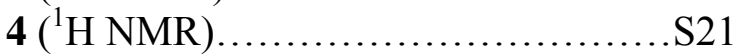

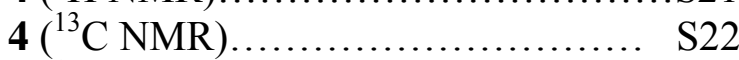

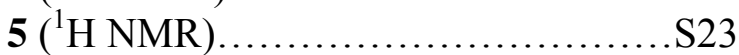

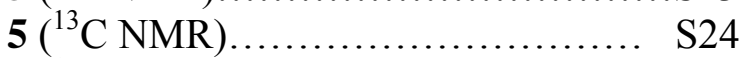

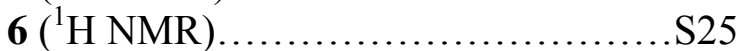

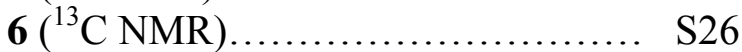

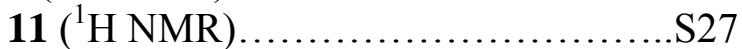

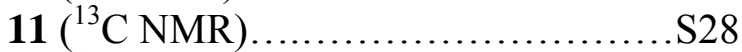

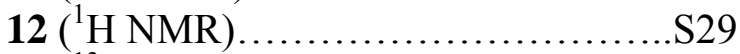

$12\left({ }^{13} \mathrm{C}\right.$ NMR $) \ldots \ldots \ldots \ldots \ldots \ldots \ldots \ldots . \quad \mathrm{S} 30$

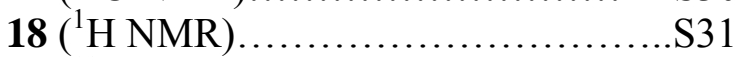

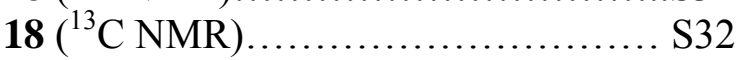




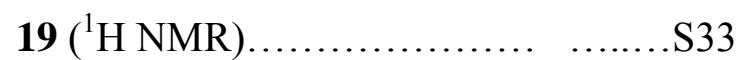

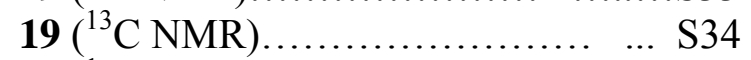

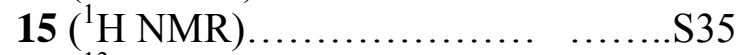

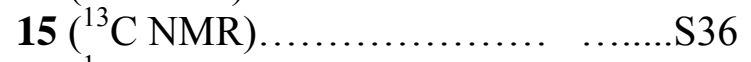

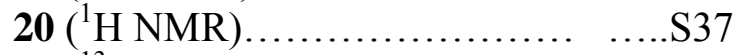

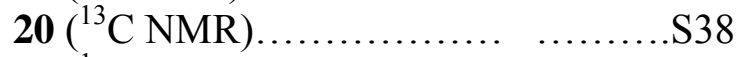

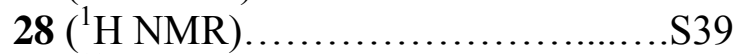

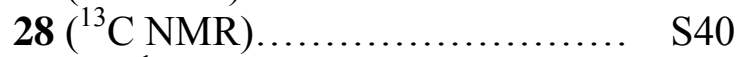

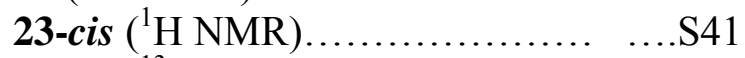

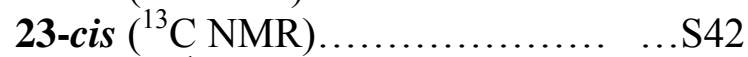

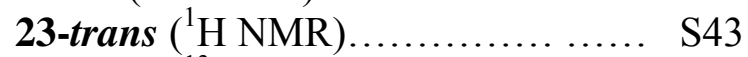

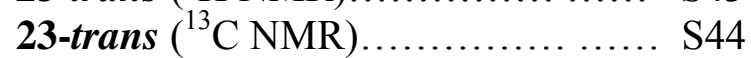

24-cis+trans $\left({ }^{1} \mathrm{H}\right.$ NMR $) \ldots \ldots \ldots \ldots . . . . . . . \quad \mathrm{S} 45$

24-cis+trans $\left({ }^{13} \mathrm{C}\right.$ NMR $) \ldots \ldots \ldots . . . . . \quad \mathrm{S} 46$

25-cis $\left({ }^{1} \mathrm{H}\right.$ NMR) ...................... . 447

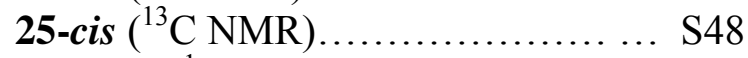

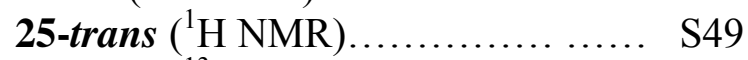

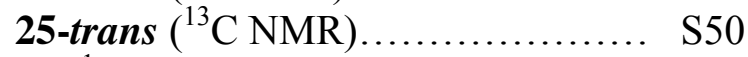

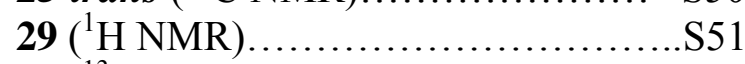

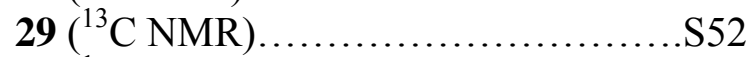

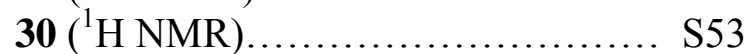

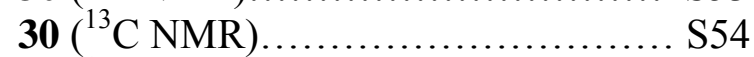

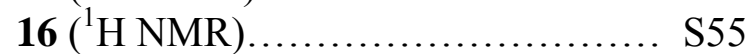

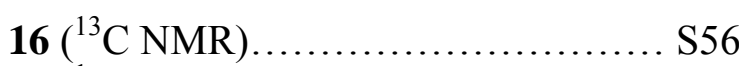

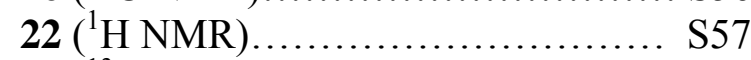

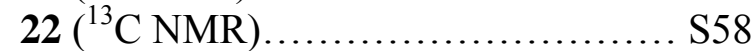

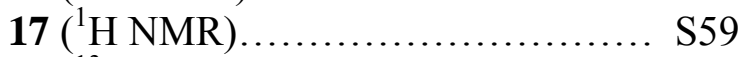

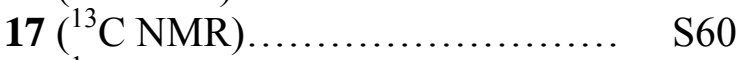

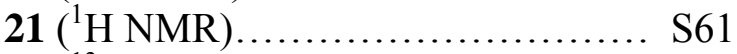

$21\left({ }^{13} \mathrm{C}\right.$ NMR $) \ldots \ldots \ldots \ldots \ldots \ldots \ldots . . . \ldots \ldots 2$

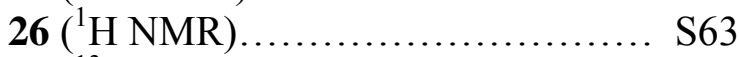

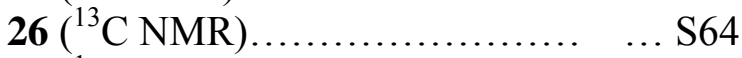

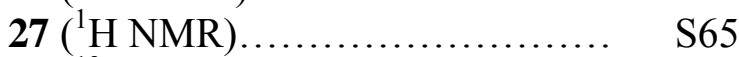

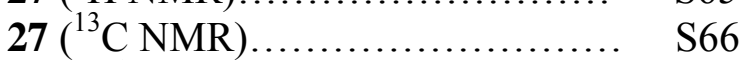

33-E $\left({ }^{1} \mathrm{H}\right.$ NMR $) \ldots \ldots \ldots \ldots \ldots \ldots \ldots \ldots$. 667

33-E $\left({ }^{13} \mathrm{C}\right.$ NMR $) \ldots \ldots \ldots \ldots \ldots \ldots \ldots . \quad \mathrm{S} 68$

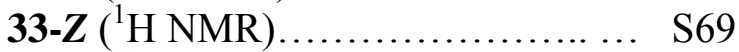

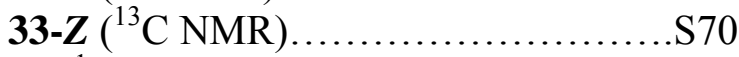

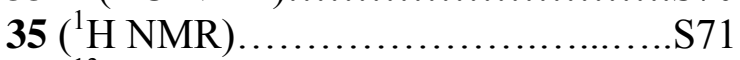

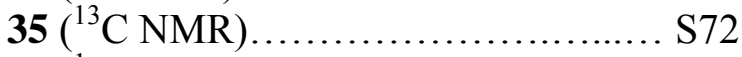

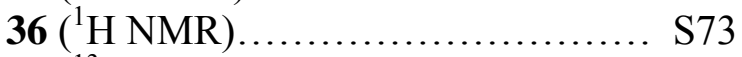

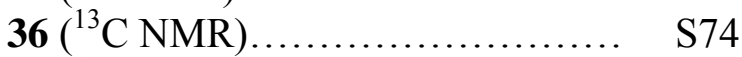

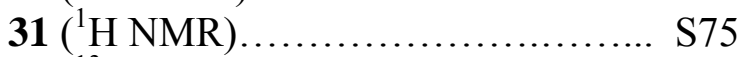

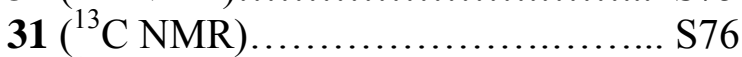

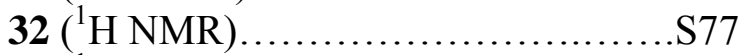

$32\left({ }^{1} \mathrm{H}\right.$ NMR $) \ldots \ldots \ldots \ldots \ldots \ldots \ldots \ldots . . \ldots \ldots$ 


\section{Experimental section}

General experimental procedures: All reactions were carried out under an argon atmosphere. Air or water-sensitive reagents were transferred using Schlenk techniques. NMR spectra were recorded (BRUKER $300 \mathrm{MHz},{ }^{1} \mathrm{H}$, and $75 \mathrm{MHz},{ }^{13} \mathrm{C}$ ) in $\mathrm{CDCl}_{3}$ (which also provided the lock signal at $\delta=7.26$ and $\delta=77.16 \mathrm{ppm}$ ). IR spectra were recorded on a Perkin Elmer FT-IR system using diamond window Dura SamplIR. Mass spectra were determined with Micromass LCT. Silica gel $(36-70 \mu \mathrm{m})$ was used for flash chromatography. TLC plates (Merck $60 \quad \mathrm{~F}_{254}$ aluminum sheets) were visualized by ultraviolet and spraying with phosphomolybdic acid (5\% in ethanol) or $\mathrm{KMnO}_{4}(5 \%$ in water). THF and ether were distilled under argon from sodium/benzophenone prior to use. Dichloromethane was distilled from $\mathrm{CaH}_{2}$ under argon prior to use.

Typical procedure for the preparation of 1,1-dichloro alkenes (method A): DBU (15.0 $\mathrm{mL}, 99.8 \mathrm{mmol})$ was added dropwise to a solution of the aldehyde $(45.0 \mathrm{mmol})$ in chloroform $(9.0 \mathrm{~mL}, 111.9 \mathrm{mmol})$. The reaction mixture was stirred at $\mathrm{rt}$ for $5 \mathrm{~h}$ then $\mathrm{Ac}_{2} \mathrm{O}(13.0 \mathrm{~mL}$, $138.5 \mathrm{mmol}$ ) was added. The solution was stirred for $16 \mathrm{~h}$. The mixture was diluted with chloroform and washed with $2 \mathrm{~N} \mathrm{HCl}$. The organic phases were dried over $\mathrm{Na}_{2} \mathrm{SO}_{4}$ and concentrated in vacuum. The residue was dissolved in $\mathrm{AcOH}(45.0 \mathrm{~mL}, 789.1 \mathrm{mmol})$ and $\mathrm{Zn}$ powder $(6.0 \mathrm{~g}, 91.8 \mathrm{mmol})$ was added and the mixture was warmed to $50{ }^{\circ} \mathrm{C}$ for $1 \mathrm{~h}$. After cooling to $\mathrm{rt}$, the reaction mixture was neutralized by $2 \mathrm{~N} \mathrm{NaOH}$ and extracted with $\mathrm{CH}_{2} \mathrm{Cl}_{2}$. The combined organic layers were dried over $\mathrm{Na}_{2} \mathrm{SO}_{4}$, concentrated in vacuum and distilled off with a Büchi oven, yielding the desired 1,1-dichloro-1-alkene.<smiles>COc1ccc(C=C(Cl)Cl)cc1</smiles>

1,1-Dichloro-2-(4-methoxyphenyl)ethane (2):

Prepared according to method A from $p$-anisaldehyde. Distillation afforded $8.4 \mathrm{~g}$ (93\% overall) of 1,1-dichloro-1-alkene 2 as a colorless oil.

IR (film) 2956, 2836, 1604, 1508, 1250, 1176, $1031 \mathrm{~cm}^{-1} ;{ }^{1} \mathrm{H}$ NMR $\left(300 \mathrm{MHz}, \mathrm{CDCl}_{3}\right) \delta_{\mathrm{H}}$ $3.81(\mathrm{~s}, 3 \mathrm{H}), 6.78(\mathrm{~s}, 1 \mathrm{H}), 6.89(\mathrm{dd}, J=2.1,6.6 \mathrm{~Hz}, 2 \mathrm{H}), 7.49(\mathrm{dd}, J=2.1,6.6 \mathrm{~Hz}, 2 \mathrm{H}) ;{ }^{13} \mathrm{C}$ NMR $\left(75 \mathrm{MHz}, \mathrm{CDCl}_{3}\right) \delta_{\mathrm{C}} 55.3,113.9,118.8,126.0,128.6,130.4,159.6 ; \mathrm{MS}(\mathrm{EI}) \mathrm{m} / \mathrm{z} 20$ $\left(\mathrm{M}^{+},{ }^{37} \mathrm{Cl}\right), 202\left(\mathrm{M}^{+},{ }^{35} \mathrm{Cl}\right), 189(\mathrm{M}-\mathrm{Me}), 187(\mathrm{M}-\mathrm{Me}), 161,159,137,131,123,89$; Anal. Calcd. for $\mathrm{C}_{9} \mathrm{H}_{8} \mathrm{Cl}_{2} \mathrm{O}$ : C 53.23; H 3.97, found C 53.47; H 3.92.<smiles>Fc1ccc(C=C(Cl)Cl)cc1</smiles>

\section{1,1-Dichloro-2-(4-fluorophenyl)ethane (4):}

Prepared according to method A from 4-fluorobenzaldehyde. Distillation afforded $7.8 \mathrm{~g}$ (79\% overall) of 1,1-dichloro-1-alkene 4 as a colorless oil.

IR (film) 3037, 2359, 1602, 1506, 1231, 1160, $910 \mathrm{~cm}^{-1} ;{ }^{1} \mathrm{H}$ NMR $\left(300 \mathrm{MHz}, \mathrm{CDCl}_{3}\right) \delta_{\mathrm{H}} 6.82$ (s, 1H), 7.03-7.10 (m, 2H), 7.49-7.55 (m, 3H); ${ }^{13} \mathrm{C} \mathrm{NMR}\left(75 \mathrm{MHz}, \mathrm{CDCl}_{3}\right) \delta_{\mathrm{C}} 115.5(\mathrm{~d}, J=$ $21.3 \mathrm{~Hz}), 120.9,127.4,129.5(\mathrm{~d}, J=3.8 \mathrm{~Hz}), 130.5(\mathrm{~d}, J=8.3 \mathrm{~Hz}), 162.3(\mathrm{~d}, J=247.6 \mathrm{~Hz})$; MS (EI) m/z $192\left(\mathrm{M}^{+},{ }^{37} \mathrm{Cl}\right), 190\left(\mathrm{M}^{+},{ }^{35} \mathrm{Cl}\right), 167,155(\mathrm{M}-\mathrm{Cl}), 125,123,120,109,95$; Anal. calcd. for $\mathrm{C}_{8} \mathrm{H}_{5} \mathrm{Cl}_{2} \mathrm{~F}$ : C 50.30; $\mathrm{H} 2.64$; found C: 50.23; $\mathrm{H} 2.48$. 
Typical procedure for the preparation of 1,1-dichloro alkenes (method B): The aldehyde (50.0 mmol) and $\mathrm{CCl}_{4}(10 \mathrm{~mL}, 103.3 \mathrm{mmol})$ were added to a suspension of Al foil $(1.835 \mathrm{~g}$, $70.4 \mathrm{mmol})$ and $\mathrm{PbBr}_{2}(208 \mathrm{mg}, 0.6 \mathrm{~mol} \%)$ in DMF $(200 \mathrm{~mL})$. A strong exothermic reaction occurred and $\mathrm{Al}$ was consumed within $1 \mathrm{~h}$. The reaction mixture was quenched with water and extracted with $\mathrm{Et}_{2} \mathrm{O}$. After extraction, the combined organic layers were dried over $\mathrm{Na}_{2} \mathrm{SO}_{4}$ and concentrated in vacuum. The residue was dissolved in pyridine (12 mL, 149.2 $\mathrm{mmol})$ and $\mathrm{Ac}_{2} \mathrm{O}(8.4 \mathrm{~mL}, 88.8 \mathrm{mmol})$ was added. The reaction mixture was stirred for $4 \mathrm{~h}$, then hydrolyzed by $2 \mathrm{~N} \mathrm{HCl}$. The solution was extracted with $\mathrm{CH}_{2} \mathrm{Cl}_{2}$. The combined organic layers were dried over $\mathrm{Na}_{2} \mathrm{SO}_{4}$ and concentrated in vacuum. The solid residue was dissolved in $\mathrm{AcOH}(40 \mathrm{~mL}, 700.1 \mathrm{mmol})$ and $\mathrm{Zn}$ powder $(6.51 \mathrm{~g}, 99.6 \mathrm{mmol})$ was added. The reaction mixture was warmed at $50{ }^{\circ} \mathrm{C}$ for $1 \mathrm{~h}$. The solution was neutralized by $2 \mathrm{~N} \mathrm{NaOH}$ and extracted with $\mathrm{CH}_{2} \mathrm{Cl}_{2}$. The combined organic layers were dried over $\mathrm{Na}_{2} \mathrm{SO}_{4}$ and concentrated in vacuum. The residue was distilled off with a Büchi oven, yielding the expected 1,1-dichloro-1-alkene.<smiles>ClC(Cl)=Cc1ccccc1</smiles>

\section{(2,2-dichlorovinyl)benzene (1):}

Prepared according to method B from benzaldehyde. Distillation afforded $7.33 \mathrm{~g}$ (87\% overall) of 1,1-dichloro-1-alkene $\mathbf{1}$ as a colorless oil.

IR (film) 3058, 3027, 1608, 1446, $900 \mathrm{~cm}^{-1} ;{ }^{1} \mathrm{H}$ NMR $\left(300 \mathrm{MHz}, \mathrm{CDCl}_{3}\right) \delta_{\mathrm{H}} 6.58(\mathrm{~s}, 1 \mathrm{H})$, $7.34(\mathrm{~m}, 3 \mathrm{H}), 7.52(\mathrm{~m}, 2 \mathrm{H}) ;{ }^{13} \mathrm{C}$ NMR $\left(75 \mathrm{MHz}, \mathrm{CDCl}_{3}\right) \delta_{\mathrm{C}} 121.1,128.60,128.69,128.77$; MS (EI) m/z $173\left(\mathrm{M}^{+},{ }^{37} \mathrm{Cl}\right), 171\left(\mathrm{M}^{+},{ }^{35} \mathrm{Cl}\right)$; Anal. calcd. for $\mathrm{C}_{10} \mathrm{H}_{10} \mathrm{Cl}_{2} \mathrm{O}_{2}$ : C 55.53; $\mathrm{H}$ 3.49; found C 54.84; H 3.52 .<smiles>CC(=O)c1ccc(C=C(Cl)Cl)cc1</smiles>

\section{1,1-Dichloro-2-(4-methoxycarbonylphenyl)ethane (3):}

Prepared according to method B from 4-methoxycarbonylbenzaldehyde. Distillation afforded $2.20 \mathrm{~g}(80 \%$ overall) of 1,1-dichloro-1-alkene 3 as a slightly yellow solid

m.p.: 57-58 ${ }^{\circ} \mathrm{C}$; IR (solid) 2952, 1715, 1597, 1438, 1272, $1115 \mathrm{~cm}^{-1} ;{ }^{1} \mathrm{H}$ NMR $(300 \mathrm{MHz}$, $\left.\mathrm{CDCl}_{3}\right) \delta_{\mathrm{H}} 3.93(\mathrm{~s}, 3 \mathrm{H}), 6.90(\mathrm{~s}, 1 \mathrm{H}), 7.60(\mathrm{dd}, J=1.8,6.3 \mathrm{~Hz}, 2 \mathrm{H}), 8.03(\mathrm{dd}, J=1.8,6.6 \mathrm{~Hz}$, $2 \mathrm{H}) ;{ }^{13} \mathrm{C} \mathrm{NMR}\left(75 \mathrm{MHz}, \mathrm{CDCl}_{3}\right) \delta_{\mathrm{C}} 52.3,123.3,127.8,128.6,129.1,129.7,137.7,160.3$; MS (EI) $\mathrm{m} / \mathrm{z} 232\left(\mathrm{M}^{+},{ }^{37} \mathrm{Cl}\right), 230\left(\mathrm{M}^{+},{ }^{35} \mathrm{Cl}\right), 212,201,199,165,163,136,121,101$; HRMS (DCI) $\mathrm{m} / \mathrm{z}$ calcd. for $\mathrm{C}_{10} \mathrm{H}_{9} \mathrm{Cl}_{2} \mathrm{O}_{2}[\mathrm{M}+\mathrm{H}]^{+}$234.9920, found 234.9915 .<smiles>COc1ccc(C=C(Cl)Cl)c(OC)c1</smiles>

\section{1,1-Dichloro-2-(2,4-dimethoxyphenyl)ethane (5):}

Prepared according to method B from 2,4-dimethoxybenzaldehyde. Distillation afforded 7.70 $\mathrm{g}(65 \%$ overall $)$ of 1,1-dichloro-1-alkene 5 as a colorless oil.

IR (film) 2940, 2837, 1605, 1575, 1498, 1455, 1293, 1264, 1207, 1159, 1121, $1032 \mathrm{~cm}^{-1} ;{ }^{1} \mathrm{H}$ $\operatorname{NMR}\left(300 \mathrm{MHz}, \mathrm{CDCl}_{3}\right) \delta_{\mathrm{H}} 3.81(\mathrm{~s}, 3 \mathrm{H}), 3.83(\mathrm{~s}, 3 \mathrm{H}), 6.43(\mathrm{~d}, J=2.4 \mathrm{~Hz}, 1 \mathrm{H}), 6.50(\mathrm{dd}, J=$ $2.4,8.7 \mathrm{~Hz}, 1 \mathrm{H}), 7.02(\mathrm{~s}, 1 \mathrm{H}), 7.70(\mathrm{~d}, J=8.6 \mathrm{~Hz}, 1 \mathrm{H}) ;{ }^{13} \mathrm{C} \mathrm{NMR}\left(75 \mathrm{MHz}, \mathrm{CDCl}_{3}\right) \delta_{\mathrm{C}} 55.4$, $55.5,98.1,104.2,115.3,118.9,123.3,129.7,158.0,161.1 ; \mathrm{MS}(\mathrm{EI}) \mathrm{m} / \mathrm{z} 234\left(\mathrm{M}^{+},{ }^{37} \mathrm{Cl}\right), 232$ 
$\left(\mathrm{M}^{+},{ }^{35} \mathrm{Cl}\right), 198(\mathrm{M}-\mathrm{Cl}), 182,167,155,121$; HRMS (DCI) $\mathrm{m} / \mathrm{z}$ calcd. for $\mathrm{C}_{10} \mathrm{H}_{11} \mathrm{Cl}_{2} \mathrm{O}_{2}[\mathrm{M}+\mathrm{H}]^{+}$ 233.0137, found 233.0139.

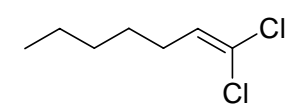

\section{1,1-dichlorohept-1-ene (6):}

Prepared according to method B from hexanal. Distillation afforded $2.70 \mathrm{~g}$ (49.5\% overall) of 1,1-dichloro-1-alkene 6 as a colorless oil.

IR (film) 2957, 2926, 2858, 1621, 1458, $882 \mathrm{~cm}^{-1} ;{ }^{1} \mathrm{H}$ NMR $\left(300 \mathrm{MHz}, \mathrm{CDCl}_{3}\right) \delta_{\mathrm{H}} 0.90$ (m, $3 \mathrm{H}), 1.31(\mathrm{~m}, 4 \mathrm{H}), 1.41(\mathrm{~m}, J=7.1 \mathrm{~Hz}, 2 \mathrm{H}), 2.16(\mathrm{q}, J=7.3 \mathrm{~Hz}, 2 \mathrm{H}), 5.85(\mathrm{t}, J=7.5 \mathrm{~Hz}, 1 \mathrm{H})$; ${ }^{13} \mathrm{C}$ NMR $\left(75 \mathrm{MHz}, \mathrm{CDCl}_{3}\right) \delta_{\mathrm{C}} 14.1,22.6,28.0,29.7,31.4,119.8,130.3 ; \mathrm{MS}(\mathrm{EI}) \mathrm{m} / \mathrm{z} 168$ $\left(\mathrm{M}^{+},{ }^{37} \mathrm{Cl}\right), 166\left(\mathrm{M}^{+},{ }^{35} \mathrm{Cl}\right)$; HRMS (DCI) $\mathrm{m} / \mathrm{z}$ calcd. for $\mathrm{C}_{7} \mathrm{H}_{12} \mathrm{Cl}_{2}[\mathrm{M}]^{+} 166.0316$, found 166.0315 .

Typical procedure for stereoselective mono coupling: A terminal alkene $(1.2 \mathrm{mmol})$ was added to a solution of 9-BBN $(0.5 \mathrm{M}$ in THF, $2.4 \mathrm{~mL}, 1.2 \mathrm{mmol})$. The solution was stirred at $\mathrm{rt}$ for $1 \mathrm{~h}$. A solution of the 1,1-dichloro-1-alkene $(1.0 \mathrm{mmol})$ in THF $(2 \mathrm{~mL})$, was added followed by $\mathrm{Pd}_{2}(\mathrm{dba})_{3}(23.1 \mathrm{mg}, 2.5 \mathrm{~mol} \%)$, XantPhos $(28.5 \mathrm{mg}, 5 \mathrm{~mol} \%)$, KF (180 mg, 3.0 mmol) and $\mathrm{K}_{3} \mathrm{PO}_{4}(635 \mathrm{mg}, 3.0 \mathrm{mmol}$ ) (method A). Alternatively, CsF (454.4 mg, 3.0 mmol)and $\mathrm{Cs}_{2} \mathrm{CO}_{3}(979.6 \mathrm{mg}, 3.0 \mathrm{mmol})$ were used instead of $\mathrm{KF}$ and $\mathrm{K}_{3} \mathrm{PO}_{4}$ (method $\mathrm{B}$ ). The solution was refluxed until completion (see Table 3 for reaction times). After cooling to $\mathrm{rt}$, the reaction mixture was quenched with water and extracted with $\mathrm{CH}_{2} \mathrm{Cl}_{2}$. The combined organic layers were dried over $\mathrm{Na}_{2} \mathrm{SO}_{4}$ and concentrated in vacuum. The residue was purified by column chromatography, affording the mono coupled product.<smiles>COc1ccc(/C=C(\Cl)CCCc2ccccc2)cc1</smiles>

\section{(Z)-2-Chloro-1-(4-methoxyphenyl)-5-phenylpent-1-ene (11):}

Prepared according to typical procedure from allylbenzene (495 $\mu \mathrm{L}, 4.17 \mathrm{mmol})$ and 1,1dichloro-1-alkene 2 (609 $\mathrm{mg}, 3.00 \mathrm{mmol})$. Purified by column chromatography (heptane $/ \mathrm{CH}_{2} \mathrm{Cl}_{2}, 85 / 15$ ) afforded 1 as a colorless solid (741 mg, 86\%, method A; $758 \mathrm{mg}$, $88 \%$, method B).

m.p.: 95-96 ${ }^{\circ} \mathrm{C}$; IR (solid) 2931, 2360, 1605, 1508, 1247, 1176, $1032 \mathrm{~cm}^{-1} ;{ }^{1} \mathrm{H} \mathrm{NMR}\left(\mathrm{CDCl}_{3}\right.$, $300 \mathrm{MHz}) \delta_{\mathrm{H}} 2.00(\mathrm{~m}, 2 \mathrm{H}), 2.49(\mathrm{t}, J=7.2 \mathrm{~Hz}, 2 \mathrm{H}), 2.67(\mathrm{t}, J=7.5 \mathrm{~Hz}, 2 \mathrm{H}), 3.81(\mathrm{~s}, 3 \mathrm{H})$, $6.39(\mathrm{~s}, 1 \mathrm{H}), 6.88(\mathrm{dd}, J=2.1,6.6 \mathrm{~Hz}, 2 \mathrm{H}), 7.20(\mathrm{~d}, J=6.9 \mathrm{~Hz}, 2 \mathrm{H}), 7.25-7.32(\mathrm{~m}, 3 \mathrm{H}), 7.57$ $(\mathrm{dd}, J=2.1,6.9 \mathrm{~Hz}, 2 \mathrm{H}) ;{ }^{13} \mathrm{C}$ NMR $\left(75 \mathrm{MHz}, \mathrm{CDCl}_{3}\right) \delta_{\mathrm{C}} 29.1,34.6,40.5,55.2,113.5,124.1$, $125.9,127.7,128.3,128.5,130.3,132.5,141.8,158.8$; MS (EI) $\mathrm{m} / \mathrm{z} 288\left(\mathrm{M}^{+},{ }^{37} \mathrm{Cl}\right), 286\left(\mathrm{M}^{+}\right.$, $\left.{ }^{35} \mathrm{Cl}\right), 181,159,147,145,131,121,115$; Anal. calcd. for $\mathrm{C}_{18} \mathrm{H}_{19} \mathrm{ClO}: \mathrm{C} 75.38$; $\mathrm{H} \mathrm{6.68}$, found C 75.32; H 6.86 .<smiles>CC(=O)c1ccc(/C=C(\Cl)[Hg]c2ccccc2)cc1</smiles>

(Z)-2-Chloro-1-(4-methoxycarbonylphenyl)-5-phenylpent-1-ene (18):

Prepared according to typical procedure from allylbenzene $(165 \mu \mathrm{L}, 1.39 \mathrm{mmol})$ and $1,1-$ dichloro-1-alkene 3 (230 mg, $1.00 \mathrm{mmol})$. Purification by column chromatography 
(heptane $/ \mathrm{CH}_{2} \mathrm{Cl}_{2}, 7 / 3$ ) yielded 18 as a colorless oil $(210 \mathrm{mg}, 65 \%$, method $\mathrm{A} ; 235 \mathrm{mg}, 73 \%$, method B).

IR (film) 2925, 1718, 1606, 1433, 1274, 1181, $1106 \mathrm{~cm}^{-1} ;{ }^{1} \mathrm{H}$ NMR $\left(300 \mathrm{MHz}, \mathrm{CDCl}_{3}\right) \delta_{\mathrm{H}}$ $2.01(\mathrm{~m}, 2 \mathrm{H}), 2.54(\mathrm{t}, J=7.5 \mathrm{~Hz}, 2 \mathrm{H}), 2.69(\mathrm{t}, J=7.5 \mathrm{~Hz}, 2 \mathrm{H}), 3.91(\mathrm{~s}, 3 \mathrm{H}), 6.49(\mathrm{~s}, 1 \mathrm{H})$, 7.19-7.23 (m, 3H), 7.28-7.33 (m, 2H), $7.65(\mathrm{dd}, J=1.8,6.6 \mathrm{~Hz}, 2 \mathrm{H}), 8.01(\mathrm{dd}, J=1.8,6.6$ $\mathrm{Hz}, 2 \mathrm{H}) ;{ }^{13} \mathrm{C} \mathrm{NMR}\left(75 \mathrm{MHz}, \mathrm{CDCl}_{3}\right) \delta_{\mathrm{C}} 29.0,34.6,40.6,52.1,124.0,125.9,128.40,128.42$, 128.8, 129.4, 136.9, 139.6, 141.5, 166.8; MS (EI) m/z $316\left(\mathrm{M}^{+},{ }^{37} \mathrm{Cl}\right), 314\left(\mathrm{M}^{+},{ }^{35} \mathrm{Cl}\right), 285$, 283, 279 (M-Cl), 249, 247; HRMS (DCI) m/z calcd. for $\mathrm{C}_{19} \mathrm{H}_{19} \mathrm{ClO}_{2}[\mathrm{M}]^{+} 314.1073$, found 314.1066 .<smiles>Cl/C(=C\c1ccccc1)c1ccccc1</smiles>

\section{(Z)-2-Chloro-1,5-diphenylpent-1-ene (15)}

Prepared according to typical procedure from allylbenzene $(165 \mu \mathrm{L}, 1.39 \mathrm{mmol})$ and $1,1-$ dichloro-1-alkene 1 (173 mg, $1.0 \mathrm{mmol}$ ). Purification by column chromatography (heptane) afforded 15 as a colorless oil (170 mg, 69\%; method A; $197 \mathrm{mg}, 80 \%$, method B).

IR (film) 2929, 1494, 1446, 1092, 906, $729 \mathrm{~cm}^{-1} ;{ }^{1} \mathrm{H}$ NMR $\left(300 \mathrm{MHz}, \mathrm{CDCl}_{3}\right) \delta_{\mathrm{H}} 2.01(\mathrm{~m}$, 2H), $2.52(\mathrm{t}, J=7.5 \mathrm{~Hz}, 2 \mathrm{H}), 2.68(\mathrm{t}, J=7.5 \mathrm{~Hz}, 2 \mathrm{H}), 6.46(\mathrm{~s}, 1 \mathrm{H}), 7.09-7.38(\mathrm{~m}, 8 \mathrm{H}), 7.56-$ $7.61(\mathrm{~m}, 2 \mathrm{H}) ;{ }^{13} \mathrm{C}$ NMR $\left(75 \mathrm{MHz} \mathrm{CDCl}_{3}\right) \delta_{\mathrm{C}} 29.1,34.6,40.5,124.8,125.9,127.5,128.1$, 128.4, 128.5, 129.0, 134.5, 135.1, 141.8; MS (EI) m/z $258\left(\mathrm{M}^{+},{ }^{37} \mathrm{Cl}\right), 256\left(\mathrm{M}^{+},{ }^{35} \mathrm{Cl}\right), 221(\mathrm{M}-$ $\mathrm{Cl}), 129,115,104$; HRMS (EI) m/z calcd. for $\mathrm{C}_{17} \mathrm{H}_{17} \mathrm{Cl}[\mathrm{M}]^{+} 256.1019$, found 256.1010.<smiles>Fc1ccc(/C=C(\Cl)[Hg]c2ccccc2)cc1</smiles>

\section{(Z)-2-Chloro-1-(4-fluorophenyl)-5-phenylpent-1-ene (19):}

Prepared according to typical procedure from allylbenzene $(165 \mu \mathrm{L}, 1.39 \mathrm{mmol})$ and 1,1dichloro-1-alkene 4 (190 mg, $1.00 \mathrm{mmol})$. Purification by column chromatography (heptane) afforded 19 as a colorless oil (225 mg, 82\%, method A; $186 \mathrm{mg}, 68 \%$, method B).

IR (film) 2930, 1602, 1506, 1453, 1226, 1159, $1104 \mathrm{~cm}^{-1} ;{ }^{1} \mathrm{H}$ NMR $\left(300 \mathrm{MHz}, \mathrm{CDCl}_{3}\right) \delta_{\mathrm{H}}$ 1.95-2.05 (m, 2H), $2.50(\mathrm{t}, J=7.2 \mathrm{~Hz}, 2 \mathrm{H}), 2.67(\mathrm{t}, J=6.9 \mathrm{~Hz}, 2 \mathrm{H}), 6.41(\mathrm{~s}, 1 \mathrm{H}), 6.97-7.06$ $(\mathrm{m}, 2 \mathrm{H}), 7.17-7.32(\mathrm{~m}, 5 \mathrm{H}), 7.53-7.59(\mathrm{~m}, 2 \mathrm{H}) ;{ }^{13} \mathrm{C} \mathrm{NMR}\left(75 \mathrm{MHz}, \mathrm{CDCl}_{3}\right) \delta_{\mathrm{C}} 29.1,34.6$, 40.4, 115.1 (d, $J=21.3 \mathrm{~Hz}), 123.6,125.9,128.4$, 128.45, 130.6, 130.7, 131.1, 131.15, 131.2, 134.3, 141.7, $161.9(\mathrm{~d}, J=245.9 \mathrm{~Hz})$; MS (EI) m/z $276\left(\mathrm{M}^{+},{ }^{37} \mathrm{Cl}\right), 274\left(\mathrm{M}^{+},{ }^{35} \mathrm{Cl}\right), 239(\mathrm{M}-$ $\mathrm{Cl}$ ), 104; HRMS (EI) m/z calcd. for $\mathrm{C}_{17} \mathrm{H}_{16} \mathrm{ClF}[\mathrm{M}]^{+} 274.0925$, found 274.0932.

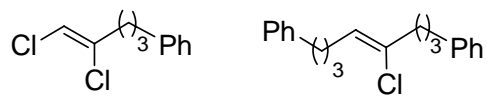

\section{(Z)-1,2-Dichloro-5-phenylpent-1-ene (29) and (Z) 5-chloro-1,8-diphenyloct-4-ene (30):}

Prepared according to typical procedure from allylbenzene $(660 \mu \mathrm{L}, 5.96 \mathrm{mmol})$ and trichloroethylene 9 (130 mg, $0.99 \mathrm{mmol})$. Purification by column chromatography (heptane) afforded the mono coupled product $29(16 \mathrm{mg}, 29 \%)$ and the bis coupled product $30(61 \mathrm{mg}$, $21 \%$ ) as colorless oils.

\section{Data for 29:}

IR (film) 2939, 1614, 1496, 1453, 1098, 869, $747 \mathrm{~cm}^{-1} ;{ }^{1} \mathrm{H}$ NMR $\left(300 \mathrm{MHz}, \mathrm{CDCl}_{3}\right) \delta_{\mathrm{H}} 1.87-$ $1.97(\mathrm{~m}, 2 \mathrm{H}), 2.40(\mathrm{t}, J=7.3 \mathrm{~Hz}, 2 \mathrm{H}), 2.62(\mathrm{t}, J=7.5 \mathrm{~Hz}, 2 \mathrm{H}), 6.13(\mathrm{~s}, 1 \mathrm{H}), 7.14-7.22(\mathrm{~m}$, 
3H), 7.25-7.32 (m, 2H); ${ }^{13} \mathrm{C}$ NMR $\left(75 \mathrm{MHz}, \mathrm{CDCl}_{3}\right) \delta_{\mathrm{C}} 28.6,34.4,36.9,113.8,126.0,128.4$, 130.6, 137.1, 138.1, 141.2; MS (EI) m/z $216\left(\mathrm{M}^{+},{ }^{37} \mathrm{Cl}\right), 214\left(\mathrm{M}^{+},{ }^{35} \mathrm{Cl}\right), 205,179(\mathrm{M}-\mathrm{Cl}), 143$, 128, 115, 105; Anal.calcd. for $\mathrm{C}_{11} \mathrm{H}_{12} \mathrm{Cl}_{2}$ : C 61.42; H 5.62; found C 61.66; H 5.80.

Data for 30:

IR (film) 2927, 1602, 1495, 1452, 908, $732 \mathrm{~cm}^{-1} ;{ }^{1} \mathrm{H}$ NMR (300 MHz, $\left.\mathrm{CDCl}_{3}\right) \delta_{\mathrm{H}} 1.66-1.74$ $(\mathrm{m}, 2 \mathrm{H}), 1.83-1.93(\mathrm{~m}, 2 \mathrm{H}), 2.19-2.26(\mathrm{~m}, 2 \mathrm{H}), 2.32-2.37(\mathrm{~m}, 2 \mathrm{H}), 2.58-2.66(\mathrm{~m}, 4 \mathrm{H}), 5.47(\mathrm{t}$ $J=6.9 \mathrm{~Hz}, 1 \mathrm{H}), 7.17-7.20(\mathrm{~m}, 5 \mathrm{H}), 7.27-7.31(\mathrm{~m}, 5 \mathrm{H}) ;{ }^{13} \mathrm{C} \mathrm{NMR}\left(75 \mathrm{MHz}, \mathrm{CDCl}_{3}\right) \delta_{\mathrm{C}} 28.2$, 29.0, 30.5, 34.6, 35.5, 38.9, 125.5, 125.7, 125.8, 128.31, 128.35, 128.4, 128.5, 134.7, 141.9, 142.3; MS (EI) m/z $300\left(\mathrm{M}^{+},{ }^{37} \mathrm{Cl}\right), 298\left(\mathrm{M}^{+},{ }^{35} \mathrm{Cl}\right), 263(\mathrm{M}-\mathrm{Cl}), 194,185,171,157,144,128$, 116, 106; Anal. calcd. for. $\mathrm{C}_{20} \mathrm{H}_{23} \mathrm{Cl}$ : $\mathrm{C} 80.38 ; \mathrm{H} 7.76$; found $\mathrm{C} 79.66 ; \mathrm{H} 7.78$<smiles>COc1ccc(/C=C/c2ccccc2)c(OC)c1</smiles>

(Z)-2-Chloro-1-(2,4-dimethoxyphenyl)-5-phenylpent-1-ene (20):

Prepared according to typical procedure from allylbenzene $(165 \mu \mathrm{L}, 1.39 \mathrm{mmol})$ and $1,1-$ dichloro-1-alkene 5 (233 mg, $0.99 \mathrm{mmol})$. Reaction time: $44 \mathrm{~h}$. Purification by column chromatography (heptane $/ \mathrm{CH}_{2} \mathrm{Cl}_{2}, 7 / 3$ ) afforded 20 as a colorless oil (260 mg, 82\%, method A; $181 \mathrm{mg}, 57 \%$, method B).

IR (film) 2944, 1607, 1577, 1499, 1453, 1288, 1262, 1157, 1128, 1118, $1032 \mathrm{~cm}^{-1} ;{ }^{1} \mathrm{H}$ NMR $\left(300 \mathrm{MHz}, \mathrm{CDCl}_{3}\right) \delta_{\mathrm{H}} 1.94-2.04(\mathrm{~m}, 2 \mathrm{H}), 2.52(\mathrm{t}, J=6.9 \mathrm{~Hz}, 2 \mathrm{H}), 2.68(\mathrm{t}, J=7.5 \mathrm{~Hz}, 2 \mathrm{H})$, $3.80(\mathrm{~s}, 3 \mathrm{H}), 3.82(\mathrm{~s}, 3 \mathrm{H}), 6.44(\mathrm{~d}, J=2.4 \mathrm{~Hz}, 1 \mathrm{H}), 6.50(\mathrm{dd}, J=2.4,8.4 \mathrm{~Hz}, 1 \mathrm{H}), 6.60(\mathrm{~s}$, 1H), 7.16-7.32 (m, 5H), $7.78(\mathrm{~d}, J=8.4 \mathrm{~Hz}, 1 \mathrm{H}) ;{ }^{13} \mathrm{C} \mathrm{NMR}\left(75 \mathrm{MHz}, \mathrm{CDCl}_{3}\right) \delta_{\mathrm{C}} 29.2,34.6$, 40.4, 55.4, 55.5, 98.2, 103.9, 117.0, 119.4, 125.8, 128.25, 128.3, 128.6, 130.5, 133.2, 158.0, 160.3; MS (EI) m/z $318\left(\mathrm{M}^{+},{ }^{37} \mathrm{Cl}\right), 316\left(\mathrm{M}^{+},{ }^{35} \mathrm{Cl}\right), 211,177,151,115,103,91$; HRMS (DCI) $\mathrm{m} / \mathrm{z}$ calcd. for $\mathrm{C}_{19} \mathrm{H}_{21} \mathrm{ClO}_{2}[\mathrm{M}]^{+} 316.1230$, found 316.1244 .<smiles>COc1ccc(/C=C(\Cl)[Hg]OCc2ccccc2)cc1</smiles>

\section{(Z)-2-Chloro-5-benzyloxy-1-(4-methoxyphenyl)pent-1-ene (16):}

Prepared according to typical procedure from allylbenzylether $(165 \mu \mathrm{L}, 1.08 \mathrm{mmol})$ and 1,1dichloro-1-alkene 2 (203 mg, $1.00 \mathrm{mmol})$. Purification by column chromatography (heptane $/ \mathrm{CH}_{2} \mathrm{Cl}_{2}, 7 / 3$ ) afforded 16 as a light yellow oil (263 mg, 83\%, method A; 250mg, $79 \%$, method B).

IR (film) 2926, 2853, 1606, 1509, 1248, 1177, 1101, $1030 \mathrm{~cm}^{-1} ;{ }^{1} \mathrm{H}$ NMR (300 MHz, $\left.\mathrm{CDCl}_{3}\right)$ $\delta_{\mathrm{H}} 1.92-2.01(\mathrm{~m}, 2 \mathrm{H}), 2.59(\mathrm{t}, J=7.5 \mathrm{~Hz}, 2 \mathrm{H}), 3.53(\mathrm{t}, J=6.3 \mathrm{~Hz}, 2 \mathrm{H}), 3.81(\mathrm{~s}, 3 \mathrm{H}) 4.51(\mathrm{~s}$, $2 \mathrm{H}), 6.40(\mathrm{~s}, 1 \mathrm{H}), 6.87(\mathrm{dd}, J=1.8,6.9 \mathrm{~Hz}, 2 \mathrm{H}), 7.25-7.35(\mathrm{~m}, 5 \mathrm{H}), 7.53(\mathrm{dd}, J=2.1,6.9 \mathrm{~Hz}$, $2 \mathrm{H}) ;{ }^{13} \mathrm{C}$ NMR $\left(75 \mathrm{MHz}, \mathrm{CDCl}_{3}\right) \delta_{\mathrm{C}} 27.7,37.8,55.2,68.8,73.0,113.5,124.2,127.6,127.7$, 128.4, 130.2, 132.2, 138.5, 158.8; MS (EI) m/z $316\left(\mathrm{M}^{+},{ }^{35} \mathrm{Cl}\right), 227,225,207,181,172,159$, 145, 115; Anal. calcd. for $\mathrm{C}_{19} \mathrm{H}_{21} \mathrm{ClO}_{2}$ : C 72.03; H 6.68; found C 71.71; H 6.99.<smiles>CCCCCCC/C(Cl)=C/c1ccc(OC)cc1</smiles>

(Z)-2-Chloro-1-(4-methoxyphenyl)non-1-ene (17): 
Prepared according to typical procedure from hept-1-ene $(170 \mu \mathrm{L}, 1.28 \mathrm{mmol})$ and 1,1 dichloro-1-alkene 2 (203 $\mathrm{mg}, 1.00 \mathrm{mmol})$. Purification by column chromatography (heptane $\left./ \mathrm{CH}_{2} \mathrm{Cl}_{2}, 85 / 15\right)$ afforded 17 as a colorless oil $(223 \mathrm{mg}, 83 \%$, method A; $164 \mathrm{mg}$, $61 \%$, method B).

IR (film) 2925, 2854, 1606, 1508, 1248, 1177, $1034 \mathrm{~cm}^{-1} ;{ }^{1} \mathrm{H}$ NMR $\left(300 \mathrm{MHz}, \mathrm{CDCl}_{3}\right) \delta_{\mathrm{H}}$ 0.86-0.91 (m, 3H), 1.27-1.34 (m, 8H), 1.59-1.69 (m, 2H), $2.45(\mathrm{t}, J=7.2 \mathrm{~Hz}, 2 \mathrm{H}), 3.81(\mathrm{~s}$, $3 \mathrm{H}), 6.39(\mathrm{~s}, 1 \mathrm{H}), 6.85-6.89(\mathrm{~m}, 2 \mathrm{H}), 7.53-7.58(\mathrm{~m}, 2 \mathrm{H}) ;{ }^{13} \mathrm{C} \mathrm{NMR}\left(75 \mathrm{MHz}, \mathrm{CDCl}_{3}\right) \delta_{\mathrm{C}} 14.1$, 22.6, 27.7, 28.6, 29.1, 31.8, 55.2, 113.5, 123.5, 127.9, 130.2, 133.3, 158.7; MS (EI) m/z 268 $\left(\mathrm{M}^{+},{ }^{37} \mathrm{Cl}\right), 266\left(\mathrm{M}^{+},{ }^{35} \mathrm{Cl}\right), 183,181,145,121,115,103$; HRMS (DCI) $\mathrm{m} / \mathrm{z}$ calcd. for $\mathrm{C}_{16} \mathrm{H}_{23} \mathrm{ClO}[\mathrm{M}]^{+}$266.1437, found 266.1437.<smiles>CCCCCCC/C(Cl)=C/c1ccc(OC)cc1OC</smiles>

\section{(Z)-2-Chloro-1-(2,4-dimethoxyphenyl)non-1-ene (21):}

Prepared according to typical procedure from hept-1-ene $(170 \mu \mathrm{L}, 1.28 \mathrm{mmol})$ and 1,1dichloro-1-alkene 5 (233 mg, $1.00 \mathrm{mmol})$. Purification by column chromatography (heptane $/ \mathrm{CH}_{2} \mathrm{Cl}_{2}, 7 / 3$ ) afforded 21 as a slightly yellow oil (248 mg, 80\%, method A; $245 \mathrm{mg}$, $79 \%$, method B).

IR (film) 2925, 2854, 1608, 1578, 1458, 1289, 1263, 1206, 1157, 1128, $1035 \mathrm{~cm}^{-1} ;{ }^{1} \mathrm{H}$ NMR $\left(300 \mathrm{MHz}, \mathrm{CDCl}_{3}\right) \delta_{\mathrm{H}} 0.87-0.91(\mathrm{~m}, 3 \mathrm{H}), 1.24-1.36(\mathrm{~m}, 8 \mathrm{H}), 1.60-1.69(\mathrm{~m}, 2 \mathrm{H}), 2.48(\mathrm{t}, J=$ $7.5 \mathrm{~Hz}, 2 \mathrm{H}), 3.80(\mathrm{~s}, 3 \mathrm{H}), 3.82(\mathrm{~s}, 3 \mathrm{H}), 6.43(\mathrm{~d}, J=2.4 \mathrm{~Hz}, 1 \mathrm{H}), 6.50$ (dd, $J=2.1,8.7 \mathrm{~Hz}$, $1 \mathrm{H}), 6.57(\mathrm{~s}, 1 \mathrm{H}), 7.77(\mathrm{~d}, J=8.7 \mathrm{~Hz}, 1 \mathrm{H}) ;{ }^{13} \mathrm{C} \mathrm{NMR}\left(75 \mathrm{MHz}, \mathrm{CDCl}_{3}\right) \delta_{\mathrm{C}} 14.1,22.6,27.7$, 28.6, 29.0, 31.8, 55.3, 55.5, 98.1, 100.0, 103.8, 117.1, 118.6, 130.4, 133.9, 158.0, 160.2; MS (EI) $\mathrm{m} / \mathrm{z} 298\left(\mathrm{M}^{+},{ }^{37} \mathrm{Cl}\right), 296\left(\mathrm{M}^{+},{ }^{35} \mathrm{Cl}\right), 261(\mathrm{M}-\mathrm{Cl}), 211,175,151,138,121,115$; HRMS (DCI) $\mathrm{m} / \mathrm{z}$ calcd. for $\mathrm{C}_{17} \mathrm{H}_{25} \mathrm{ClO}_{2}[\mathrm{M}]^{+} 296.1543$, found 296.1554 .<smiles>CCCCCCC/C(Cl)=C/c1ccc(F)cc1</smiles>

\section{(Z)-2-Chloro-1-(4-fluorophenyl)non-1-ene (22):}

Prepared according to typical procedure from hept-1-ene $(170 \mu \mathrm{L}, 1.28 \mathrm{mmol})$ and 1,1 dichloro-1-alkene 4 (190 mg, $1.00 \mathrm{mmol}$ ). Purification by column chromatography (heptane) afforded 22 as a slightly yellow oil (200 mg, 74\%, method A; $235 \mathrm{mg}, 87 \%$, method B).

IR (film) 2925, 2855, 1602, 1506, $1229 \mathrm{~cm}^{-1} ;{ }^{1} \mathrm{H}$ NMR $\left(300 \mathrm{MHz}, \mathrm{CDCl}_{3}\right) \delta_{\mathrm{H}}$ 0.87-0.91 (m, $3 \mathrm{H}), 1.26-1.36(\mathrm{~m}, 8 \mathrm{H}), 1.60-1.69(\mathrm{~m}, 2 \mathrm{H}), 2.47(\mathrm{t}, J=7.2 \mathrm{~Hz}, 2 \mathrm{H}), 6.42(\mathrm{~s}, 1 \mathrm{H}), 6.99-7.06$ $(\mathrm{m}, 2 \mathrm{H}), 7.53-7.60(\mathrm{~m}, 2 \mathrm{H}) ;{ }^{13} \mathrm{C}$ NMR $\left(75 \mathrm{MHz}, \mathrm{CDCl}_{3}\right) \delta_{\mathrm{C}} 14.1,22.6,27.6,28.6,29.0,31.8$, $41.1,115.0(\mathrm{~d}, J=21.3 \mathrm{~Hz}), 123.0,130.6(\mathrm{~d}, J=8.2 \mathrm{~Hz}), 131.3,135.0,161.8(\mathrm{~d}, J=245.4$ $\mathrm{Hz})$; MS (EI) m/z $256\left(\mathrm{M}^{+},{ }^{37} \mathrm{Cl}\right), 254\left(\mathrm{M}^{+},{ }^{35} \mathrm{Cl}\right), 147,135,133,109 ;$ HRMS (DCI) m/z calcd. for $\mathrm{C}_{15} \mathrm{H}_{20} \mathrm{ClF}[\mathrm{M}]^{+}$254.1237, found 254.1228.<smiles>CCCCCC/C=C(\Cl)CCCCCCC</smiles>

\section{(Z)-7-Chloro-tetradec-6-ene (27):}

Prepared according to typical procedure from hept-1-ene $(170 \mu \mathrm{L}, 1.28 \mathrm{mmol})$ and 1,1 dichloro-1-alkene 6 (167 mg, $1.00 \mathrm{mmol})$. Purification by column chromatography (heptane) afforded 27 as a slightly yellow oil (170 mg, 74\%, method A; $149 \mathrm{mg}, 65 \%$, method B). 
IR (film) 2955, 2923, 2855, 2359, 2340, 1660, $1465 \mathrm{~cm}^{-1} ;{ }^{1} \mathrm{H}$ NMR $\left(300 \mathrm{MHz}, \mathrm{CDCl}_{3}\right) \delta_{\mathrm{H}}$ $0.88(\mathrm{t}, J=6.6 \mathrm{~Hz}, 3 \mathrm{H}), 0.89(\mathrm{t}, J=6.9 \mathrm{~Hz}, 3 \mathrm{H}), 1.24-1.40(\mathrm{~m}, 14 \mathrm{H}), 1.48-1.58(\mathrm{~m}, 2 \mathrm{H}), 2.15$ $(\mathrm{q}, J=7.2 \mathrm{~Hz}, 2 \mathrm{H}), 2.29(\mathrm{dt}, J=0.6,7.2 \mathrm{~Hz}, 2 \mathrm{H}), 5.43(\mathrm{t}, J=6.9 \mathrm{~Hz}, 1 \mathrm{H}) ;{ }^{13} \mathrm{C} \mathrm{NMR}(75$ $\left.\mathrm{MHz}, \mathrm{CDCl}_{3}\right) \delta_{\mathrm{C}} 14.0,14.1,22.5,22.6,27.4,28.4,28.5,29.0,31.4,31.8,39.5,125.4,134.7$; MS (EI) m/z 189, 172, 153, 135, 107; HRMS (DCI) m/z calcd. for $\mathrm{C}_{14} \mathrm{H}_{27} \mathrm{Cl}[\mathrm{M}]^{+} 230.1801$, found 230.1801 .

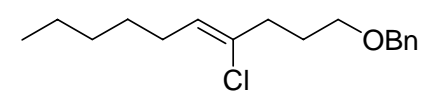

\section{(Z)-4-Chloro-1-benzyloxydec-4-ene (26):}

Prepared according to typical procedure from allylbenzylether $(180 \mu \mathrm{L}, 1.17 \mathrm{mmol})$ and 1,1 dichloro-1-alkene 6 (167 mg, $1.00 \mathrm{mmol})$. Purification by column chromatography (heptane $\left./ \mathrm{CH}_{2} \mathrm{Cl}_{2}, 85 / 15\right)$ afforded 26 as a slightly yellow oil (170 mg, 65\%, method A; 120 $\mathrm{mg}, 46 \%$, method B).

IR (film) 2924, 2854, 1453, $1102 \mathrm{~cm}^{-1} ;{ }^{1} \mathrm{H}$ NMR $\left(300 \mathrm{MHz}, \mathrm{CDCl}_{3}\right) \delta_{\mathrm{H}} 0.89(\mathrm{t}, J=6.6 \mathrm{~Hz}$, $3 \mathrm{H}), 1.21-1.40(\mathrm{~m}, 6 \mathrm{H}), 1.81-1.90(\mathrm{~m}, 2 \mathrm{H}), 2.10-2.17(\mathrm{~m}, 2 \mathrm{H}), 2.42(\mathrm{t}, J=7.2 \mathrm{~Hz}, 2 \mathrm{H}), 3.47$ $(\mathrm{t}, J=6.0 \mathrm{~Hz}, 2 \mathrm{H}), 4.49(\mathrm{~s}, 2 \mathrm{H}), 5.44(\mathrm{t}, J=6.9 \mathrm{~Hz}, 1 \mathrm{H}), 7.26-7.36(\mathrm{~m}, 5 \mathrm{H}) ;{ }^{13} \mathrm{C} \mathrm{NMR}(75$ $\left.\mathrm{MHz}, \mathrm{CDCl}_{3}\right) \delta_{\mathrm{C}} 14.0,22.5,27.5,28.3,28.4,31.4,36.1,68.2,72.9,126.2,127.5,127.6$, 128.3, 133.7, 138.5; MS (EI) m/z $280\left(\mathrm{M}^{+},{ }^{35} \mathrm{Cl}\right), 268,254$; HRMS (DCI, $\mathrm{NH}_{3}$ ) $\mathrm{m} / \mathrm{z}$ calcd. for $\mathrm{C}_{17} \mathrm{H}_{29} \mathrm{ClNO}\left[\mathrm{M}+\mathrm{NH}_{4}\right]^{+} 298.1938$, found 298.1935 .

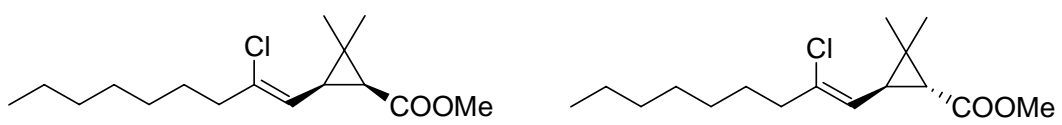

Cis (Z)-Methyl-3-(2-chloronon-1-enyl)-2,2-dimethyl-(1-cyclopropane)carboxylate (25-cis) and trans (Z)-Methyl-3-(2-chloronon-1-enyl)-2,2-dimethyl-(1-cyclopropane)carboxylate (25-trans):

Prepared according to typical procedure from hept-1-ene $(170 \mu \mathrm{L}, 1.28 \mathrm{mmol})$ and $1,1-$ dichloro-1-alkene 7 (223 mg, $1.00 \mathrm{mmol})$. Reaction time: $144 \mathrm{~h}$. Purification by column chromatography (heptane $/ \mathrm{CH}_{2} \mathrm{Cl}_{2}, 7 / 3$ ) afforded 25-cis and 25-trans (179 mg, 63\%, method A; $199 \mathrm{mg}, 70 \%$, method B) as colorless oils.

Data for 25-cis:

IR (film) 2925, 2856, 1727, 1435, 1192, $1142 \mathrm{~cm}^{-1} ;{ }^{1} \mathrm{H}$ NMR $\left(300 \mathrm{MHz}, \mathrm{CDCl}_{3}\right) \delta_{\mathrm{H}} 0.88(\mathrm{t}, J$ $=6.6 \mathrm{~Hz}, 3 \mathrm{H}), 1.24(\mathrm{~s}, 6 \mathrm{H}), 1.26-1.30(\mathrm{~m}, 8 \mathrm{H}), 1.56(\mathrm{~m}, 2 \mathrm{H}), 1.75(\mathrm{~d}, J=8.7 \mathrm{~Hz}, 1 \mathrm{H}), 2.16(\mathrm{t}$, $J=8.7 \mathrm{~Hz}, 1 \mathrm{H}), 2.35(\mathrm{t}, J=7.2 \mathrm{~Hz}, 2 \mathrm{H}), 3.65(\mathrm{~s}, 3 \mathrm{H}), 5.82(\mathrm{~d}, J=8.7 \mathrm{~Hz}, 1 \mathrm{H}) ;{ }^{13} \mathrm{C}$ NMR $(75$ $\left.\mathrm{MHz}, \mathrm{CDCl}_{3}\right) \delta_{\mathrm{C}} 14.1,14.9,22.6,27.0,27.5,28.5,29.0,31.2,31.8,32.2,39.7,51.2,119.6$, 136.9, 171.5; MS (EI) m/z $288\left(\mathrm{M}^{+},{ }^{37} \mathrm{Cl}\right), 286\left(\mathrm{M}^{+},{ }^{35} \mathrm{Cl}\right), 251(\mathrm{M}-\mathrm{Cl}), 227,129,107$; HRMS (DCI) $\mathrm{m} / \mathrm{z}$ calcd. for $\mathrm{C}_{16} \mathrm{H}_{27} \mathrm{ClO}_{2}[\mathrm{M}]^{+} 286.1700$, found 286.1709 .

Data for 25-trans:

IR (film) 2925, 2856, 1728, 1438, 1225, 1164, $1114 \mathrm{~cm}^{-1} ;{ }^{1} \mathrm{H}$ NMR $\left(300 \mathrm{MHz}, \mathrm{CDCl}_{3}\right) \delta_{\mathrm{H}}$ $0.88(\mathrm{t}, J=6.6 \mathrm{~Hz}, 3 \mathrm{H}), 0.80(\mathrm{~s}, 3 \mathrm{H}), 1.15-1.30(\mathrm{~m}, 11 \mathrm{H}), 1.50(\mathrm{~d}, J=5.4 \mathrm{~Hz}, 1 \mathrm{H}), 1.53-1.58$ $(\mathrm{m}, 2 \mathrm{H}), 2.28-2.34(\mathrm{~m}, 3 \mathrm{H}), 3.69(\mathrm{~s}, 3 \mathrm{H}), 5.19(\mathrm{~d}, J=7.8 \mathrm{~Hz}, 1 \mathrm{H}) ;{ }^{13} \mathrm{C}$ NMR $(75 \mathrm{MHz}$, $\left.\mathrm{CDCl}_{3}\right) \delta_{\mathrm{C}} 14.1,20.2,22.5,22.7,27.4,28.5,28.7,29.0,31.8,32.5,34.6,39.5,122.2,137.8$, 172.3; MS (EI) m/z $288\left(\mathrm{M}^{+},{ }^{37} \mathrm{Cl}\right), 286\left(\mathrm{M}^{+},{ }^{35} \mathrm{Cl}\right), 251,227,129,107$; HRMS (DCI) $\mathrm{m} / \mathrm{z}$ calcd. for $\mathrm{C}_{16} \mathrm{H}_{28} \mathrm{ClO}_{2}[\mathrm{M}+\mathrm{H}]^{+} 287.1778$, found 287.1778 . 
$\mathrm{ClO}_{\text {Coome }}$

cis cyclopropane)carboxylate (23-cis) and trans (Z)-Methyl-3-(2-chloro-5-phenylpent-1enyl)-2,2-dimethyl-(1-cyclopropane)carboxylate (23-trans):

Prepared according to typical procedure from allylbenzene $(165 \mu \mathrm{L}, 1.39 \mathrm{mmol})$ and 1,1 dichloro-1-alkene 7 (223 $\mathrm{mg}, 1.00 \mathrm{mmol})$. Purification by column chromatography (heptane/ $\mathrm{CH}_{2} \mathrm{Cl}_{2}, 7 / 3$ ) afforded 23-cis and 23-trans (176 mg, 58\%, method A; $240 \mathrm{mg}, 79 \%$, method B) as colorless oils.

Data for 23-cis:

IR (film) 2946, 1725, 1437, 1168, $1113 \mathrm{~cm}^{-1}$; ${ }^{1} \mathrm{H}$ NMR $\left(300 \mathrm{MHz}, \mathrm{CDCl}_{3}\right) \delta_{\mathrm{H}} 1.16(\mathrm{~s}, 3 \mathrm{H})$, $1.29(\mathrm{~s}, 3 \mathrm{H}), 1.50(\mathrm{~d}, J=3 \mathrm{~Hz}, 1 \mathrm{H}), 1.87-1.90(\mathrm{~m}, 2 \mathrm{H}), 2.32-2.37(\mathrm{~m}, 3 \mathrm{H}), 2.61(\mathrm{t}, J=7.2 \mathrm{~Hz}$, 2H), $3.69(\mathrm{~s}, 3 \mathrm{H}), 5.20(\mathrm{~d}, J=4.5 \mathrm{~Hz}, 1 \mathrm{H}), 7.16-7.20(\mathrm{~m}, 3 \mathrm{H}), 7.26-7.29(\mathrm{~m}, 2 \mathrm{H}) ;{ }^{13} \mathrm{C} \mathrm{NMR}$ $\left(75 \mathrm{MHz}, \mathrm{CDCl}_{3}\right) \delta_{\mathrm{C}} 20.2,22.5,28.6,28.9,32.5,34.6,38.8,51.6,122.8,125.9,128.3,128.4$, 137.1, 141.8, 172.2; MS (EI) m/z $306\left(\mathrm{M}^{+},{ }^{35} \mathrm{Cl}\right), 271(\mathrm{M}-\mathrm{Cl}), 247,211,155,105 ;$ HRMS (DCI) $\mathrm{m} / \mathrm{z}$ calcd. for $\mathrm{C}_{18} \mathrm{H}_{23} \mathrm{ClO}_{2}[\mathrm{M}]^{+} 306.1386$, found 306.1384 .

Data for 23-trans:

IR (film) 2950, 1724, 1436, 1191, $1138 \mathrm{~cm}^{-1} ;{ }^{1} \mathrm{H}$ NMR (300 MHz, $\left.\mathrm{CDCl}_{3}\right) \delta_{\mathrm{H}} 1.24$ (d, J=1.5 $\mathrm{Hz}, 3 \mathrm{H}), 1.25(\mathrm{~d}, J=1.5 \mathrm{~Hz}, 3 \mathrm{H}), 1.77(\mathrm{dd}, J=1.5,8.4 \mathrm{~Hz}, 1 \mathrm{H}), 1.83-1.94(\mathrm{~m}, 2 \mathrm{H}), 2.17$ (dt, $J=1.2,8.6 \mathrm{~Hz}, 1 \mathrm{H}), 2.39(\mathrm{t}, J=7.2 \mathrm{~Hz}, 2 \mathrm{H}), 2.60(\mathrm{t}, J=7.8 \mathrm{~Hz}, 2 \mathrm{H}), 3.64(\mathrm{~s}, 3 \mathrm{H}), 5.86(\mathrm{~d}, J$ $=8.6 \mathrm{~Hz}, 1 \mathrm{H}), 7.15-7.20(\mathrm{~m}, 3 \mathrm{H}), 7.25-7.30(\mathrm{~m}, 2 \mathrm{H}) ;{ }^{13} \mathrm{C} \mathrm{NMR}\left(75 \mathrm{MHz}, \mathrm{CDCl}_{3}\right) \delta_{\mathrm{C}} 14.9$, 27.0, 28.9, 29.1, 31.2, 32.2, 34.6, 39.1, 51.2, 120.3, 125.8, 128.3, 128.5, 136.3, 141.9, 171.4; MS (EI) m/z $308\left(\mathrm{M}^{+},{ }^{37} \mathrm{Cl}\right), 306\left(\mathrm{M}^{+},{ }^{35} \mathrm{Cl}\right), 271(\mathrm{M}-\mathrm{Cl}), 247,211,155,129,117$; HRMS (DCI) $\mathrm{m} / \mathrm{z}$ calcd. for $\mathrm{C}_{18} \mathrm{H}_{23} \mathrm{ClO}_{2}[\mathrm{M}]^{+} 306.1386$, found 306.1389 .

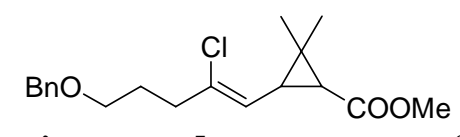

cis and trans (Z)-Methyl-3-(2-chloro-5-benzyloxypent-1-enyl)-2,2-dimethyl-(1cyclopropane)carboxylate (24):

Prepared according to typical procedure from allylbenzylether $(180 \mu \mathrm{L}, 1.17 \mathrm{mmol})$ and 1,1dichloro-1-alkene $7(223 \mathrm{mg}, 1.00 \mathrm{mmol})$. Reaction time: $161 \mathrm{~h}$. Purification by column chromatography (heptane/ $\left.\mathrm{CH}_{2} \mathrm{Cl}_{2}, 1 / 2\right)$ afforded 24 as a colorless oil (195 mg, 59\%, method $\mathrm{A} ; 215 \mathrm{mg}, 65 \%$, method $\mathrm{B}$ as a mixture of stereoisomers at cyclopropyl ring).

IR (film) 2949, 2924, 2856, 1725, 1437, $1166 \mathrm{~cm}^{-1} ;{ }^{1} \mathrm{H}$ NMR (300 MHz, $\left.\mathrm{CDCl}_{3}\right) \delta_{\mathrm{H}} 1.12(\mathrm{~s}$, $2 \mathrm{H}), 1.23(\mathrm{~d}, J=1.5 \mathrm{~Hz}, 1 \mathrm{H}), 1.26(\mathrm{~s}, 2 \mathrm{H}), 1.48(\mathrm{~d}, J=5.4 \mathrm{~Hz}, 0.7 \mathrm{H}), 1.59(\mathrm{~s}, 1 \mathrm{H}), 1.76(\mathrm{~d}, J$ $=8.4 \mathrm{~Hz}, 0.3 \mathrm{H}), 1.81-1.92(\mathrm{~m}, 2 \mathrm{H}), 2.12-2.18(\mathrm{~m}, 0.3 \mathrm{H}), 2.28-2.33(\mathrm{~m}, 0.7 \mathrm{H}), 2.40-2.50(\mathrm{~m}$, $2 \mathrm{H}), 3.45-3.50(\mathrm{~m}, 2 \mathrm{H}), 3.64(\mathrm{~s}, 1 \mathrm{H}), 3.69(\mathrm{~s}, 2 \mathrm{H}), 4.49(\mathrm{~s}, 1.3 \mathrm{H}), 4.50(\mathrm{~s}, 0.7 \mathrm{H}), 5.20(\mathrm{~d}, J=$ $8.1 \mathrm{~Hz}, 0.7 \mathrm{H}), 5.87(\mathrm{~d}, J=8.4 \mathrm{~Hz}, 0.3 \mathrm{H}), 7.30-7.35(\mathrm{~m}, 5 \mathrm{H}) ;{ }^{13} \mathrm{C} \mathrm{NMR}\left(75 \mathrm{MHz}, \mathrm{CDCl}_{3}\right) \delta_{\mathrm{C}}$ 20.2, 22.5, 27.6, 27.7, 28.5, 28.6, 32.1, 32.5, 34.5, 36.1, 36.4, 51.2, 51.6, 68.7, 69.0, 120.2, $122.9,127.5,127.6,127.65,128.4,136.9,138.4,172.2 ; \mathrm{MS}(\mathrm{EI}) \mathrm{m} / \mathrm{z} 337\left(\mathrm{M}^{+},{ }^{35} \mathrm{Cl}\right), 319$, 301, 269, 241, 211, 179; HRMS (DCI) m/z calcd. for $\mathrm{C}_{19} \mathrm{H}_{25} \mathrm{ClO}_{3}[\mathrm{M}+\mathrm{H}]^{+} 337.1571$, found 337.1576.

Typical procedure for non-selective cross-coupling of 1,1-dichloro alkenes: A terminal alkene (1.2 mmol) was added to a solution of 9-BBN (0.5 M in THF, $2.4 \mathrm{~mL}, 1.2 \mathrm{mmol})$. The solution was stirred at $\mathrm{rt}$ for $1 \mathrm{~h}$. To a solution of 1,1-dichloro-1-alkene $(1.0 \mathrm{mmol})$ in THF (2 
$\mathrm{mL}$ ) was added $\mathrm{Pd}_{2} \mathrm{Cl}_{2}$ (dppp) (16.6 mg, $2.5 \mathrm{~mol} \%$ ), $\mathrm{KF}$ (180 mg, $\left.3.0 \mathrm{mmol}\right)$ and $\mathrm{K}_{3} \mathrm{PO}_{4}(635$ $\mathrm{mg}, 3.0 \mathrm{mmol})$. The solution was refluxed until tlc shown completion. After cooling to $\mathrm{rt}$, the reaction mixture was quenched with water and extracted with $\mathrm{CH}_{2} \mathrm{Cl}_{2}$. The combined organic layers were dried over $\mathrm{Na}_{2} \mathrm{SO}_{4}$ and concentrated in vacuum. The residue was purified by column chromatography, affording both mono and bis coupled products.<smiles>COc1ccc(C=C(/C=C\c2ccccc2)/C=C\c2ccccc2)c(OC)c1</smiles>

(Z)-2-Chloro-1-(2,4-dimethoxyphenyl)-5-phenylpent-1-ene (20) and 1-(2,4dimethoxyphenyl)-5-phenyl-2-(3-phenylpropyl)pent-1-ene (28):

Prepared according to typical procedure from allylbenzene $(165 \mu \mathrm{L}, 1.39 \mathrm{mmol})$ and 1,1 dichloro-1-alkene $5(233 \mathrm{mg}, 1.00 \mathrm{mmol})$. Reaction time: $24 \mathrm{~h}$. Purification by column chromatography (heptane $\left./ \mathrm{CH}_{2} \mathrm{Cl}_{2}, 7 / 3\right)$ afforded both the mono coupled product $20(75 \mathrm{mg}$, $24 \%)$ and the bis coupled product 28 (73 $\mathrm{mg}, 18 \%)$ as colorless oils.

Data for 20:

IR (film) 2944, 1607, 1577, 1499, 1453, 1288, 1262, 1157, 1128, 1118, $1032 \mathrm{~cm}^{-1} ;{ }^{1} \mathrm{H}$ NMR $\left(300 \mathrm{MHz} \mathrm{CDCl}_{3}\right) \delta_{\mathrm{H}} 1.94-2.04(\mathrm{~m}, 2 \mathrm{H}), 2.52(\mathrm{t}, J=6.9 \mathrm{~Hz}, 2 \mathrm{H}), 2.68(\mathrm{t}, J=7.5 \mathrm{~Hz}, 2 \mathrm{H})$, $3.80(\mathrm{~s}, 3 \mathrm{H}), 3.82(\mathrm{~s}, 3 \mathrm{H}), 6.44(\mathrm{~d}, J=2.4 \mathrm{~Hz}, 1 \mathrm{H}), 6.50(\mathrm{dd}, J=2.4,8.4 \mathrm{~Hz}, 1 \mathrm{H}), 6.60(\mathrm{~s}$, $1 \mathrm{H}), 7.16-7.32(\mathrm{~m}, 5 \mathrm{H}), 7.78(\mathrm{~d}, J=8.4 \mathrm{~Hz}, 1 \mathrm{H}) ;{ }^{13} \mathrm{C}$ NMR $\left(75 \mathrm{MHz}, \mathrm{CDCl}_{3}\right) \delta_{\mathrm{C}} 29.2,34.6$, 40.4, 55.4, 55.5, 98.2, 103.9, 117.0, 119.4, 125.8, 128.25, 128.3, 128.6, 130.5, 133.2, 158.0, 160.3; MS (EI) m/z $318\left(\mathrm{M}^{+},{ }^{37} \mathrm{Cl}\right), 316\left(\mathrm{M}^{+},{ }^{35} \mathrm{Cl}\right), 211,177,151,115,103,91$; HRMS (DCI) $\mathrm{m} / \mathrm{z}$ calcd. for $\mathrm{C}_{19} \mathrm{H}_{22} \mathrm{ClO}_{2}[\mathrm{M}+\mathrm{H}]^{+} 317.1308$, found 317.1313 .

Data for 28:

IR (film) 2930, 1606, 1577, 1497, 1452, 1284, 1259, 1206, 1156, 1120, $1034 \mathrm{~cm}^{-1} ;{ }^{1} \mathrm{H}$ NMR $\left(300 \mathrm{MHz}, \mathrm{CDCl}_{3}\right) \delta_{\mathrm{H}} 1.68-1.87(\mathrm{~m}, 4 \mathrm{H}), 2.19-2.25(\mathrm{~m}, 4 \mathrm{H}), 2.55(\mathrm{t}, J=7.8 \mathrm{~Hz}, 2 \mathrm{H}), 2.65(\mathrm{t}$, $J=7.8 \mathrm{~Hz}, 2 \mathrm{H}), 3.78(\mathrm{~s}, 3 \mathrm{H}), 3.81(\mathrm{~s}, 3 \mathrm{H}), 6.24(\mathrm{~s}, 1 \mathrm{H}), 6.38(\mathrm{dd}, J=1.8,8.1 \mathrm{~Hz}, 1 \mathrm{H}), 6.44$ $(\mathrm{m}, 1 \mathrm{H}), 6.96(\mathrm{~d}, J=8.1 \mathrm{~Hz}, 1 \mathrm{H}), 7.10-7.31(\mathrm{~m}, 10 \mathrm{H}) ;{ }^{13} \mathrm{C}$ NMR $\left(75 \mathrm{MHz}, \mathrm{CDCl}_{3}\right) \delta_{\mathrm{C}} 29.8$, 29.9, 30.4, 35.7, 35.9, 36.3, 55.3, 55.4, 98.3, 103.7, 120.1, 120.7, 125.6, 128.2, 128.3, 128.4, 128.5, 130.2, 141.3, 142.4, 142.7, 158.0, 159.4; MS (EI) m/z $400\left(\mathrm{M}^{+}\right), 295,192,177,151$, 91; HRMS (DCI) $\mathrm{m} / \mathrm{z}$ calcd. for $\mathrm{C}_{28} \mathrm{H}_{32} \mathrm{O}_{2}[\mathrm{M}]^{+} 400.2402$, found 400.2398.<smiles>COc1ccc(C=C(CCCc2ccccc2)CCCc2ccccc2)cc1</smiles>

\section{2-(3-Phenylpropyl)-1-(4-methoxyphenyl)-5-phenylpent-1-ene (12):}

Prepared according to typical procedure from allylbenzene $(135 \mu \mathrm{L}, 1.13 \mathrm{mmol})$ and alkenyl chloride 2 (203 mg, $1.00 \mathrm{mmol}$. Reaction time: $22 \mathrm{~h}$. Purification by column chromatography (heptane $\left./ \mathrm{CH}_{2} \mathrm{Cl}_{2}, 85 / 15\right)$ afforded 12 as a colorless oil (170 $\mathrm{mg}, 58 \%$ ).

IR (film) 2928, 2360, 1605, 1508, 1452, 1246, 1175, $1032 \mathrm{~cm}^{-1} ;{ }^{1} \mathrm{H}$ NMR (300 MHz, CDCl $)$ $\delta_{\mathrm{H}} 1.78(\mathrm{~m}, 4 \mathrm{H}), 2.23(\mathrm{~m}, 4 \mathrm{H}), 2.61(\mathrm{~m}, 4 \mathrm{H}), 3.80(\mathrm{~s}, 3 \mathrm{H}), 6.22(\mathrm{~s}, 1 \mathrm{H}), 6.79(\mathrm{dd}, J=2.1,6.3$ $\mathrm{Hz}, 2 \mathrm{H}), 7.03-7.06(\mathrm{~m}, 2 \mathrm{H}), 7.12-7.30(\mathrm{~m}, 10 \mathrm{H}) ;{ }^{13} \mathrm{C} \mathrm{NMR}\left(75 \mathrm{MHz}, \mathrm{CDCl}_{3}\right) \delta_{\mathrm{C}} 29.9,30.1$, $35.6,35.9,36.8,55.2,113.5,125.0,125.7,128.2$, 128.4, 129.6, 130.9, 141.3, 142.2, 142.5, 
157.7; MS (EI) m/z $370\left(\mathrm{M}^{+}\right), 249,173,162,147,121 ; \mathrm{HRMS}(\mathrm{DCI}) \mathrm{m} / \mathrm{z}$ calcd. for $\mathrm{C}_{27} \mathrm{H}_{30} \mathrm{O}$ $[\mathrm{M}]^{+} 370.2297$, found 370.2300 .

Typical procedure for second coupling, leading to tri-substituted olefins: A terminal alkene $(1.2 \mathrm{mmol})$ was added to a solution of 9-BBN (0.5 M in THF, $2.4 \mathrm{~mL}, 1.2 \mathrm{mmol})$. The solution was stirred at $\mathrm{rt}$ for $1 \mathrm{~h}$. Chloro-olefin $(1.0 \mathrm{mmol})$ was added dissolved in THF (2 $\mathrm{mL})$, and $\mathrm{PdCl}_{2}(\mathrm{dppp}) \quad(5 \mathrm{~mol} \%) \quad$ or $\mathrm{Pd}_{2}(\mathrm{dba})_{3} \quad\left(\begin{array}{lll}5 & \mathrm{~mol} \%\end{array}\right)$ with 2(dicyclohexylphosphino)biphenyl (10 mol\%), KF (180 mg, $3.0 \mathrm{mmol})$ and $\mathrm{K}_{3} \mathrm{PO}_{4}(636 \mathrm{mg}$, $3.0 \mathrm{mmol}$ ) were successively added. The reaction mixture was refluxed until completion. After cooling to rt, the solution was quenched with water and extracted with $\mathrm{CH}_{2} \mathrm{Cl}_{2}$. The combined organic layers were dried over $\mathrm{Na}_{2} \mathrm{SO}_{4}$ and concentrated in vacuum. The residue was purified by column chromatography, yielding the stereoselectively tri-substituted olefin.<smiles>CCCCCCCC/C(=C\c1ccc(C(C)=O)cc1)CCCc1ccccc1</smiles>

(E)-2-Heptyl-1-(4-methoxycarbonylphenyl)-5-phenylpent-1-ene (32):

Prepared according to typical procedure from hept-1-ene $(170 \mu \mathrm{L}, 1.28 \mathrm{mmol})$ and alkenyl chloride 18 (202 $\mathrm{mg}, 0.59 \mathrm{mmol})$. Reaction time: $26 \mathrm{~h}$. Purification by column chromatography (heptane/ $\left.\mathrm{CH}_{2} \mathrm{Cl}_{2}, 7 / 3\right)$ afforded 32 as a colorless oil (160 mg, 69\%).

IR (film) 2923, 2853, 1720, 1604, 1433, 1273, 1176, $1101 \mathrm{~cm}^{-1} ;{ }^{1} \mathrm{H}$ NMR (300 MHz, CDCl $\left.{ }_{3}\right)$ $\delta_{\mathrm{H}}$ 0.85-0.89 (m, 3H), 1.23-1.28 (m, 8H), 1.43-1.46 (m, 2H), 1.80-1.90 (m, 2H), 2.19-2.25 (m, $4 \mathrm{H}), 2.67(\mathrm{t}, J=7.5 \mathrm{~Hz}, 2 \mathrm{H}), 3.90(\mathrm{~s}, 3 \mathrm{H}), 6.28(\mathrm{~s}, 1 \mathrm{H}), 7.14-7.32(\mathrm{~m}, 7 \mathrm{H}), 7.95-7.99(\mathrm{~m}, 2 \mathrm{H})$; ${ }^{13} \mathrm{C}$ NMR $\left(75 \mathrm{MHz}, \mathrm{CDCl}_{3}\right) \delta_{\mathrm{C}} 14.0,22.6,28.2,29.0,29.7,29.8,30.8,31.8,35.6,37.0,51.9$, $124.4,125.7,127.4,128.3,128.4,128.5,129.4,142.3,143.4,145.8,167.1 ; \mathrm{MS}(\mathrm{EI}) \mathrm{m} / \mathrm{z} 378$ $\left(\mathrm{M}^{+}\right), 190,175,131,129,104,91$; HRMS (EI) $\mathrm{m} / \mathrm{z}$ calcd. for $\mathrm{C}_{26} \mathrm{H}_{34} \mathrm{O}_{2}[\mathrm{M}]^{+}$378.2558, found 378.2545 .<smiles>COc1ccc(/C=C(\CCCCc2ccccc2)CCCc2ccccc2)cc1</smiles>

(E)-2-(3-Phenylpropyl)-5-benzyloxy-1-(4-methoxyphenyl)pent-1-ene (31):

Prepared according to typical procedure from allylbenzene $(165 \mu \mathrm{L}, 1.39 \mathrm{mmol})$ and alkenyl chloride 16 (172 $\mathrm{mg}, 0.54 \mathrm{mmol})$. Reaction time: $46 \mathrm{~h}$. Purification by column chromatography (heptane/ $\left.\mathrm{CH}_{2} \mathrm{Cl}_{2}, 1 / 1\right)$ afforded 31 as a colorless oil (136 mg, 69\%).

IR (film) 2929, 2850, 1605, 1507, 1452, 1245, 1174, 1100, $1029 \mathrm{~cm}^{-1} ;{ }^{1} \mathrm{H}$ NMR (300 MHz, $\left.\mathrm{CDCl}_{3}\right) \delta_{\mathrm{H}} 1.74-1.85(\mathrm{~m}, 4 \mathrm{H}), 2.21-2.29(\mathrm{~m}, 4 \mathrm{H}), 2.59(\mathrm{~d}, J=7.5 \mathrm{~Hz}, 2 \mathrm{H}), 3.49-3.54(\mathrm{~m}, 2 \mathrm{H})$, $3.80(\mathrm{~s}, 3 \mathrm{H}), 4.51(\mathrm{~s}, 2 \mathrm{H}), 6.21(\mathrm{~s}, 1 \mathrm{H}), 6.76-6.80(\mathrm{~m}, 2 \mathrm{H}), 7.13-7.35(\mathrm{~m}, 12 \mathrm{H}) ;{ }^{13} \mathrm{C}$ NMR $(75$ $\left.\mathrm{MHz}, \mathrm{CDCl}_{3}\right) \delta_{\mathrm{C}} 28.2,29.9,30.2,33.7,35.9,55.2,69.9,72.9,113.5,125.0,125.7,127.5$, 127.6, 128.2, 128.3, 128.5, 129.6, 130.9, 138.6, 141.0, 142.2, 157.7; MS (EI) m/z $400\left(\mathrm{M}^{+}\right)$, 294, 282, 176, 147, 121, 91; HRMS (DCI) m/z calcd. for $\mathrm{C}_{28} \mathrm{H}_{32} \mathrm{O}_{2}[\mathrm{M}]^{+}$400.2402, found 400.2397 .<smiles>CCCCCCC/C(=C\c1ccc(F)cc1)CCCc1ccccc1</smiles>

(Z)-2-(3-Phenylpropyl)-1-(4-fluorophenyl)non-1-ene (33-Z):

Prepared according to typical procedure from allylbenzene $(135 \mu \mathrm{L}, 1.13 \mathrm{mmol})$ and alkenyl chloride 22 (192 mg, $0.75 \mathrm{mmol}$ ). Reaction time: $95 \mathrm{~h}$. Purification by column chromatography (heptane) afforded 33-Z as a colorless oil (190 mg, 74\%). 
IR (film) 2924, 2854, 1505, $1224 \mathrm{~cm}^{-1} ;{ }^{1} \mathrm{H}$ NMR (300 MHz, $\left.\mathrm{CDCl}_{3}\right) \delta_{\mathrm{H}} 0.89(\mathrm{t}, J=6.6 \mathrm{~Hz}$, $3 \mathrm{H}), 1.24-1.31(\mathrm{~m}, 8 \mathrm{H}), 1.41-1.51(\mathrm{~m}, 2 \mathrm{H}), 1.71-1.82(\mathrm{~m}, 2 \mathrm{H}), 2.11-2.24(\mathrm{~m}, 4 \mathrm{H}), 2.58(\mathrm{t}, J=$ $7.5 \mathrm{~Hz}, 2 \mathrm{H}), 6.20(\mathrm{~s}, 1 \mathrm{H}), 6.87-6.95(\mathrm{~m}, 2 \mathrm{H}), 7.01-7.07(\mathrm{~m}, 2 \mathrm{H}), 7.12-7.29(\mathrm{~m}, 5 \mathrm{H}) ;{ }^{13} \mathrm{C} \mathrm{NMR}$ $\left(75 \mathrm{MHz}, \mathrm{CDCl}_{3}\right) \delta_{\mathrm{C}} 14.1,22.7,28.2,29.2,29.4,29.9,30.0,31.9,35.9,37.2,114.9(\mathrm{~d}, J=$ $21.2 \mathrm{~Hz}), 124.0,125.8,128.3(\mathrm{~d}, J=13.0 \mathrm{~Hz}), 130.0,130.1,134.5(\mathrm{~d}, J=3.3 \mathrm{~Hz}), 142.1$, 143.3, $161.0(\mathrm{~d}, J=244.4 \mathrm{~Hz})$; MS (EI) m/z $338\left(\mathrm{M}^{+}\right), 234,150,135,103$; Anal. calcd. for $\mathrm{C}_{24} \mathrm{H}_{31} \mathrm{~F}$ : C 85.16; H 9.23; found C 85.08; H 9.45.<smiles>CCCCCCCCC(=Cc1ccc(F)cc1)CCCP</smiles>

(E)-2-(3-phenylpropyl)-1-(4-fluorophenyl)non-1-ene (33-E):

Prepared according to typical procedure from hept-1-ene $(60 \mu \mathrm{L}, 0.44 \mathrm{mmol})$ and alkenyl chloride 19 ( $87 \mathrm{mg}, 0.32 \mathrm{mmol})$. Reaction time: $76 \mathrm{~h}$. Purification by column chromatography (heptane) afforded 33-E as a colorless oil (76 mg, 74\%).

IR (film) 2924, 2854, 1505, $1224 \mathrm{~cm}^{-1} ;{ }^{1} \mathrm{H}$ NMR (300 MHz, $\left.\mathrm{CDCl}_{3}\right) \delta_{\mathrm{H}}$ 0.85-0.89 (m, 3H), 1.24-1.26 (m, 8H), 1.36-1.46 (m, 2H), 1.78-1.88 (m, 2H), 2.14-2.22 (m, 4H), $2.66(\mathrm{~d}, J=7.8$ $\mathrm{Hz}, 2 \mathrm{H}), 6.21(\mathrm{~s}, 1 \mathrm{H}), 6.94-7.02(\mathrm{~m}, 2 \mathrm{H}), 7.10-7.32(\mathrm{~m}, 7 \mathrm{H}) ;{ }^{13} \mathrm{C} \mathrm{NMR}\left(75 \mathrm{MHz}, \mathrm{CDCl}_{3}\right) \delta_{\mathrm{C}}$ 14.1, 22.6, 28.2, 29.1, 29.7, 29.9, 30.5, 31.8, 35.6, 36.7, 114.8 (d, $J=21.3 \mathrm{~Hz}$ ), 124.0, 125.8, $128.4(\mathrm{~d}, J=9.8 \mathrm{~Hz}), 130.0,130.1,134.5(\mathrm{~d}, J=3.2 \mathrm{~Hz}), 142.5,143.4 ;$ MS (EI) $\mathrm{m} / \mathrm{z} 338$ $\left(\mathrm{M}^{+}\right), 150,135,109$, 104; HRMS (DCI) $\mathrm{m} / \mathrm{z}$ calcd. for $\mathrm{C}_{24} \mathrm{H}_{31} \mathrm{~F}[\mathrm{M}]^{+}$338.2410, found 338.2424 .

Typical procedure for Suzuki cross-coupling with phenylboronic acid 34: Alkenyl chloride (1.0 mmol.), phenylboronic acid 34 (1.2 equiv.), $\operatorname{Pd}_{2}(\mathrm{dba})_{3}(5 \mathrm{~mol} \%)$, 2(dicyclohexylphosphinyl)biphenyl (10 mol\%) KF (176 mg, $3.0 \mathrm{mmmol})$ and $\mathrm{K}_{3} \mathrm{PO}_{4}(636 \mathrm{mg}$, $3.0 \mathrm{mmol}$ ) were refluxed in THF for several hours. After cooling to $\mathrm{rt}$, the reaction mixture was quenched with water and extracted with $\mathrm{CH}_{2} \mathrm{Cl}_{2}$. The combined organic layers were dried over $\mathrm{Na}_{2} \mathrm{SO}_{4}$ and concentrated in vacuum. The residue was purified by column chromatography.

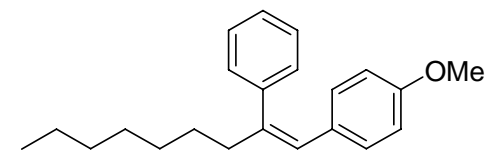

\section{(Z)-2-Phenyl-1-(4-methoxyphenyl)non-1-ene (35):}

Prepared according to typical procedure from alkenyl chloride 17 (204 mg, $0.76 \mathrm{mmol})$ and phenylboronic acid (121 mg, $1.00 \mathrm{mmol})$. Reaction time: $89 \mathrm{~h}$. Purification by column chromatography (heptane $\left./ \mathrm{CH}_{2} \mathrm{Cl}_{2}, 85 / 15\right)$ afforded 35 as a colorless oil (134 $\mathrm{mg}, 58 \%$ ).

IR (film) 2953, 2924, 2853, 1606, 1508, 1247, 1175, $1035 \mathrm{~cm}^{-1} ;{ }^{1} \mathrm{H}$ NMR (300 MHz, $\left.\mathrm{CDCl}_{3}\right)$ $\delta_{\mathrm{H}} 0.84-0.89(\mathrm{~m}, 3 \mathrm{H}), 1.24-1.41(\mathrm{~m}, 10 \mathrm{H}), 2.45(\mathrm{dt}, J=1.2,7.5 \mathrm{~Hz}, 2 \mathrm{H}), 3.71(\mathrm{~s}, 3 \mathrm{H}), 6.36(\mathrm{~s}$, $1 \mathrm{H}), 6.59-6.64(\mathrm{~m}, 2 \mathrm{H}), 6.80-6.85(\mathrm{~m}, 2 \mathrm{H}), 7.13-7.17(\mathrm{~m}, 2 \mathrm{H}), 7.23-7.33(\mathrm{~m}, 3 \mathrm{H}) ;{ }^{13} \mathrm{C} \mathrm{NMR}$ $\left(75 \mathrm{MHz}, \mathrm{CDCl}_{3}\right) \delta_{\mathrm{C}} 14.1,22.6,28.0,29.1,29.2,31.8,40.7,55.1,113.2,125.4,126.6,128.5$, 128.6, 130.0, 130.2, 141.6, 141.7, 157.8; MS (EI) m/z $308\left(\mathrm{M}^{+}\right), 224,178,165,145,121,114$; Anal. calcd. for $\mathrm{C}_{22} \mathrm{H}_{28} \mathrm{O}$ : C 85.66; $\mathrm{H}$ 9.15; found $\mathrm{C} 85.74 ; \mathrm{H} 9.39$.<smiles>COc1ccc(/C=C(\CCCc2ccccc2)c2ccccc2)cc1</smiles> 


\section{(Z)-2,5-diphenyl-1-(4-methoxyphenyl)pent-1-ene (36):}

Prepared according to typical procedure from allkenyl chloride $11(287 \mathrm{mg}, 1.00 \mathrm{mmol})$ and phenylboronic acid (145 mg, $1.19 \mathrm{mmol})$. Reaction time: $97 \mathrm{~h}$. Purification by column chromatography (heptane/ $\left.\mathrm{CH}_{2} \mathrm{Cl}_{2}, 85 / 15\right)$ afforded 36 as a colorless oil $(220 \mathrm{mg}, 66 \%)$ and some starting material 11 was recovered (75 mg, 26\%).

IR (film) 2927, 2854, 1605, 1508, 1440, 1247, 1175, $1033 \mathrm{~cm}^{-1} ;{ }^{1} \mathrm{H}$ NMR (300 MHz, $\mathrm{CDCl}_{3}$ ) $\delta_{\mathrm{H}} 1.69-1.75(\mathrm{~m}, 2 \mathrm{H}), 2.52(\mathrm{~d}, J=4.5 \mathrm{~Hz}, 2 \mathrm{H}), 2.64(\mathrm{~d}, J=4.5 \mathrm{~Hz}, 2 \mathrm{H}), 3.71(\mathrm{~s}, 3 \mathrm{H}), 6.37(\mathrm{~s}$, $1 \mathrm{H}), 6.61-6.63(\mathrm{~m}, 2 \mathrm{H}), 6.83-6.85(\mathrm{~m}, 2 \mathrm{H}), 7.14-7.17(\mathrm{~m}, 4 \mathrm{H}), 7.24-7.31(\mathrm{~m}, 6 \mathrm{H}) ;{ }^{13} \mathrm{C} \mathrm{NMR}$ $\left(75 \mathrm{MHz}, \mathrm{CDCl}_{3}\right) \delta_{\mathrm{C}} 29.6,35.4,40.2,55.1,113.2,125.7,125.9,126.8,128.2,128.4,128.5$, 128.6, 130.0, 140.9, 141.4, 142.4, 157.9; MS (EI) m/z $328\left(\mathrm{M}^{+}\right), 224,121,115 ;$ HRMS (DCI) $\mathrm{m} / \mathrm{z}$ calcd. for $\mathrm{C}_{24} \mathrm{H}_{24} \mathrm{O}[\mathrm{M}]^{+} 328.1828$, found 328.1833 . 


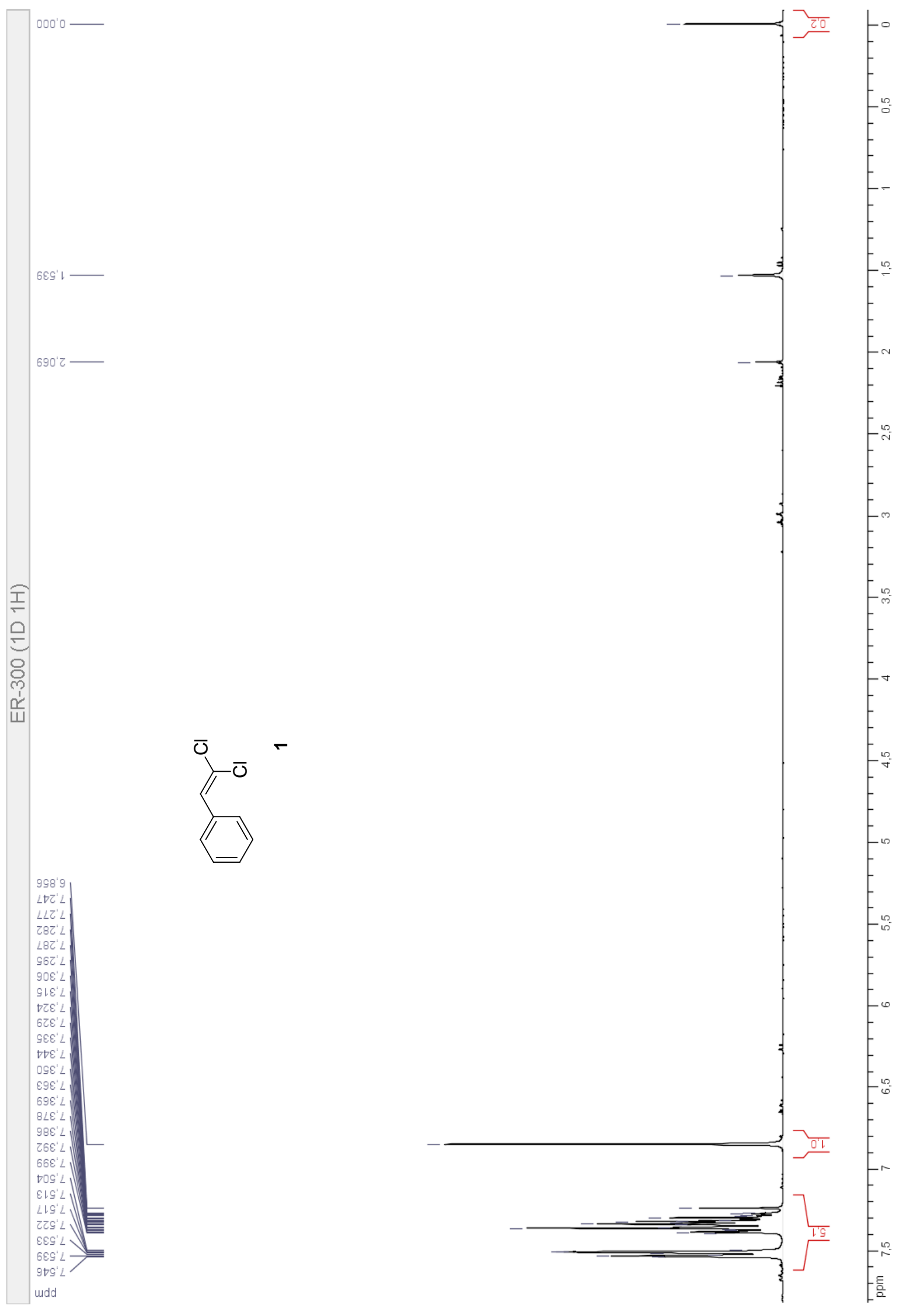




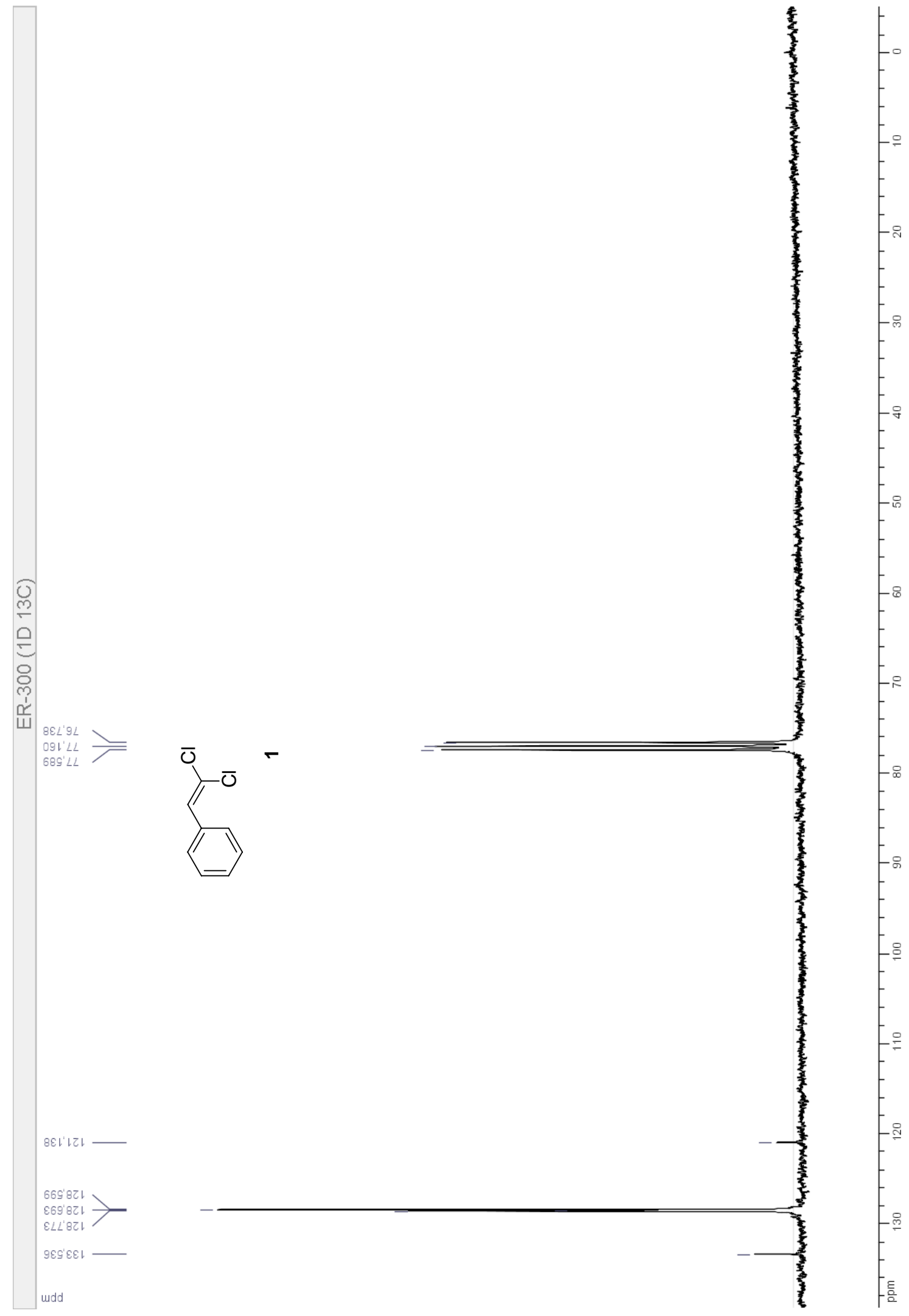




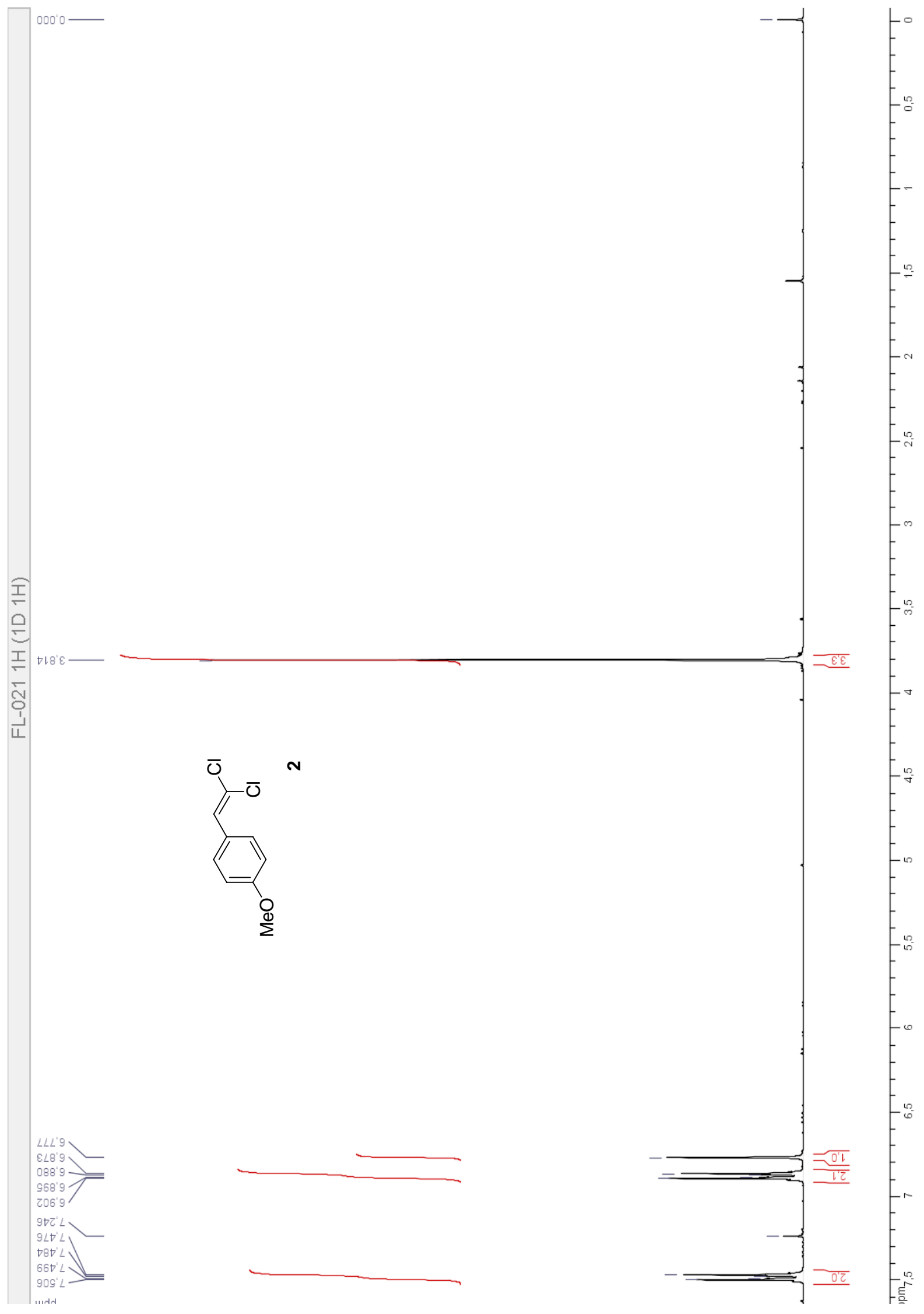




$$
\forall=
$$




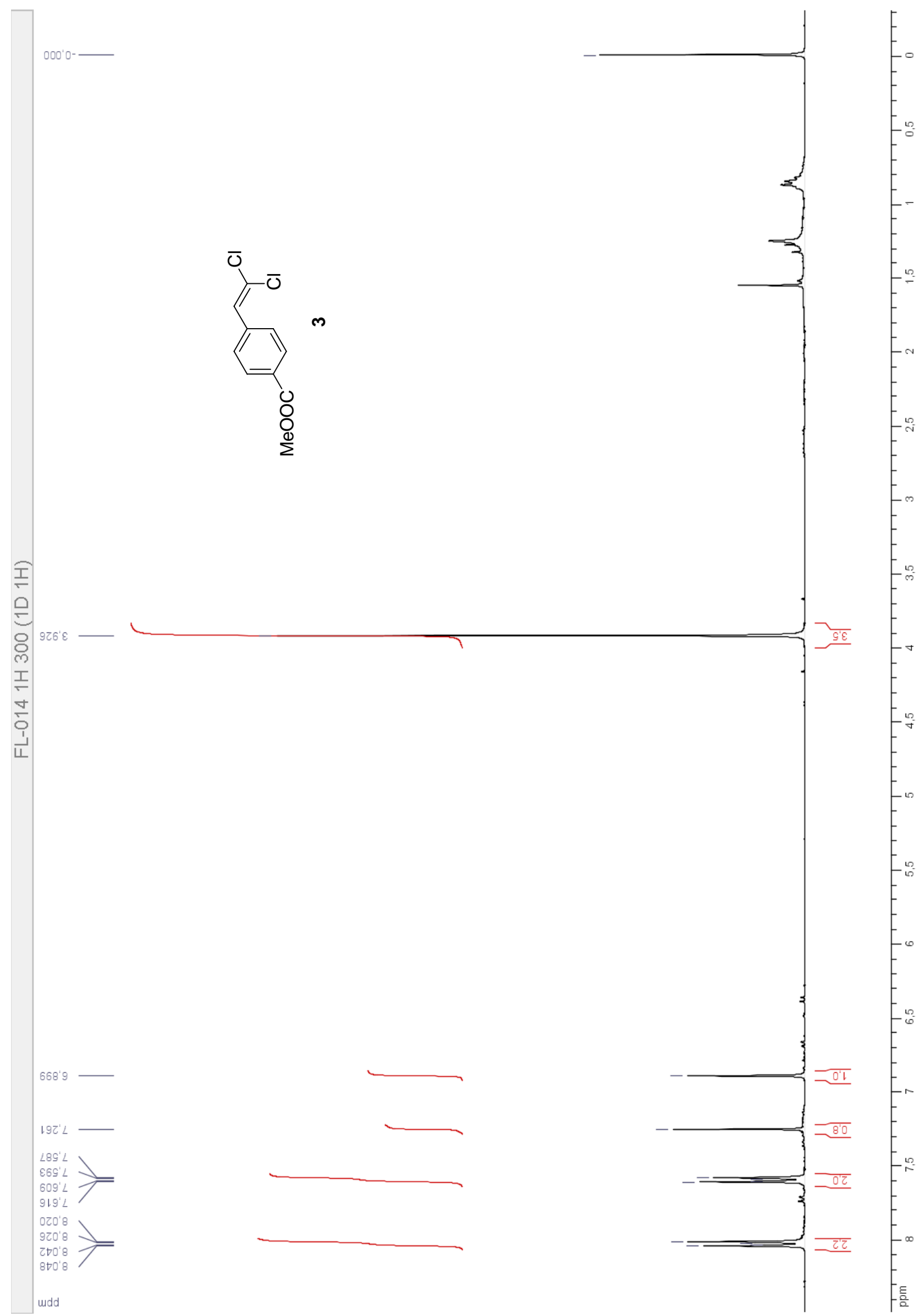




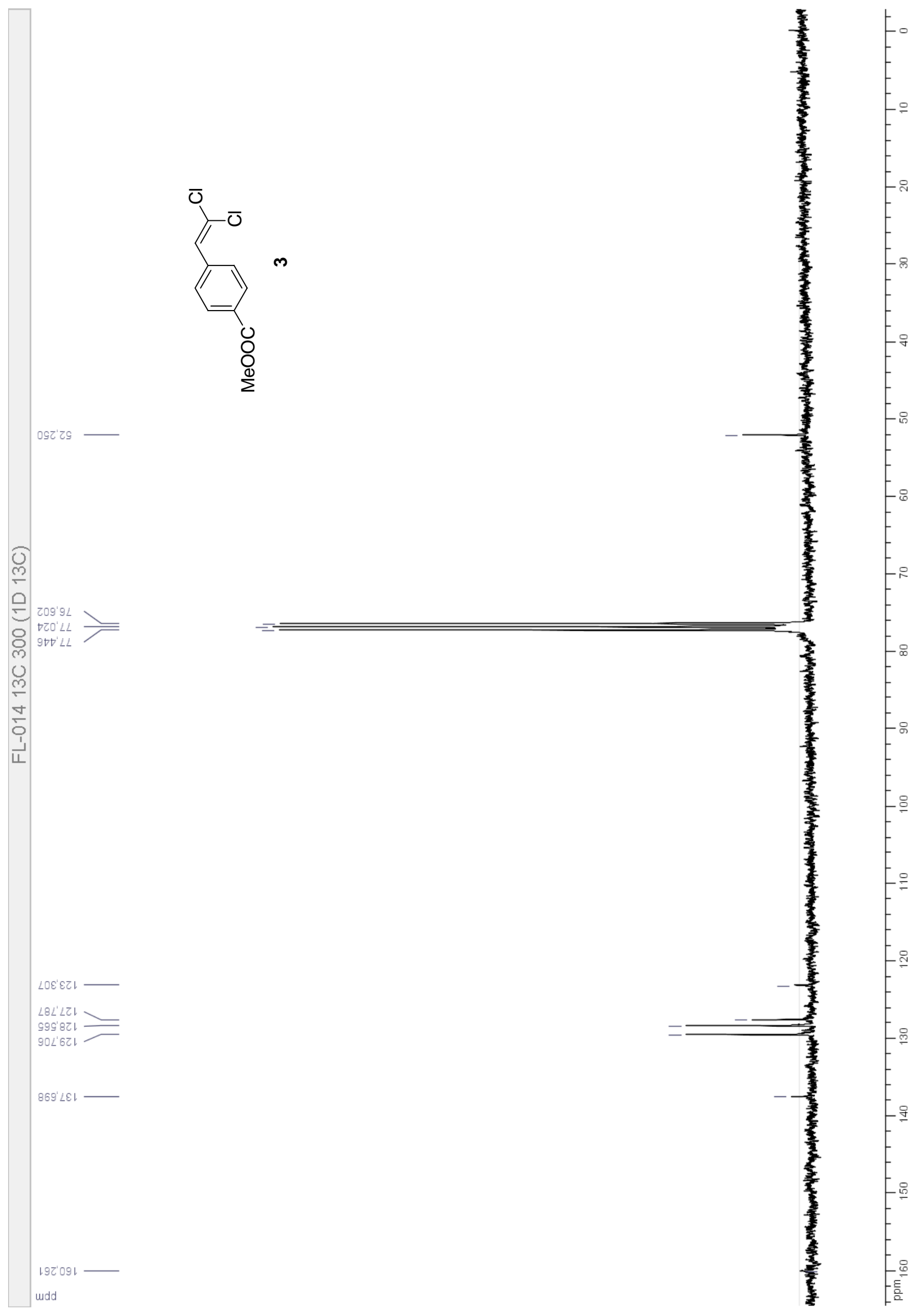

S20 

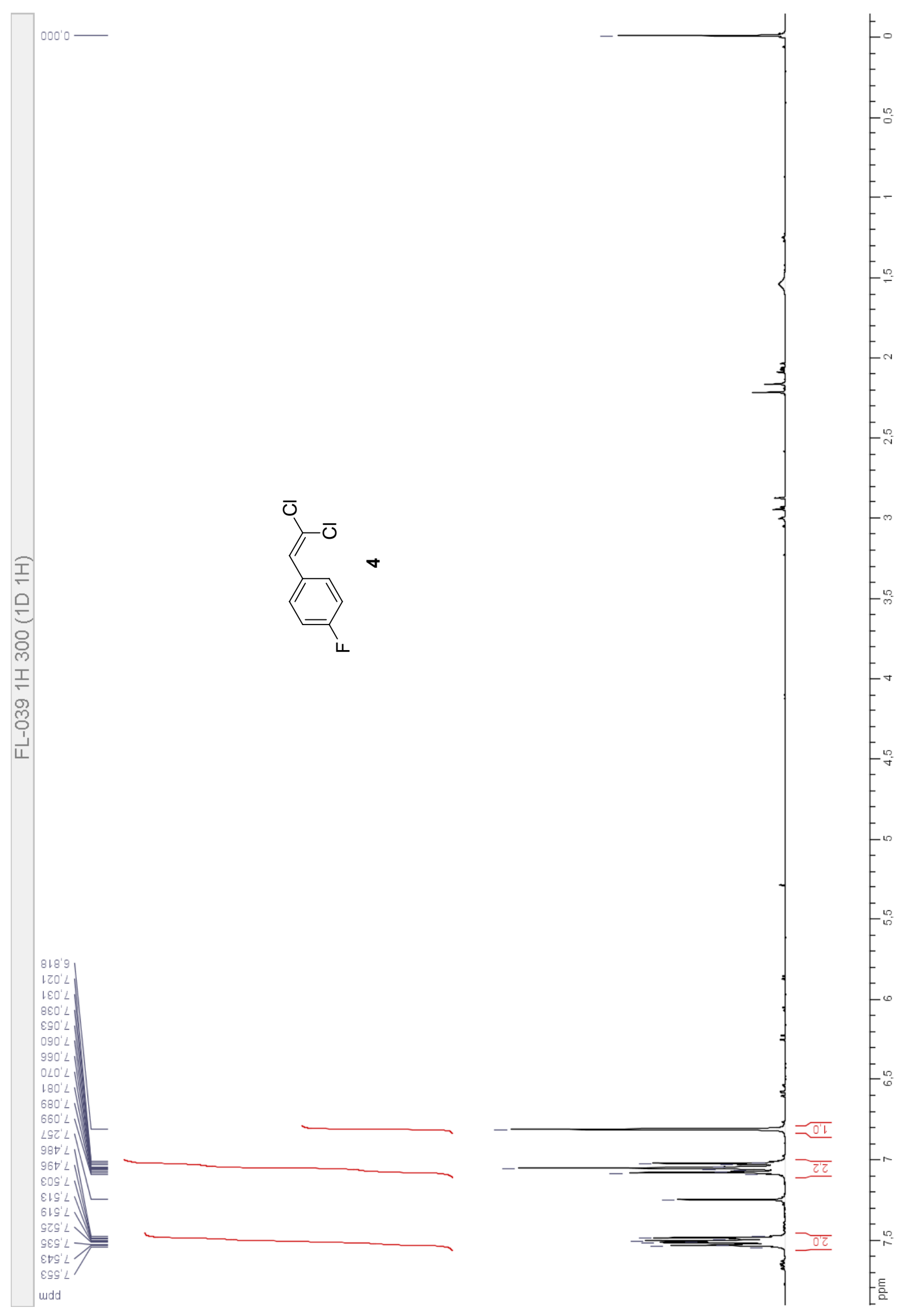


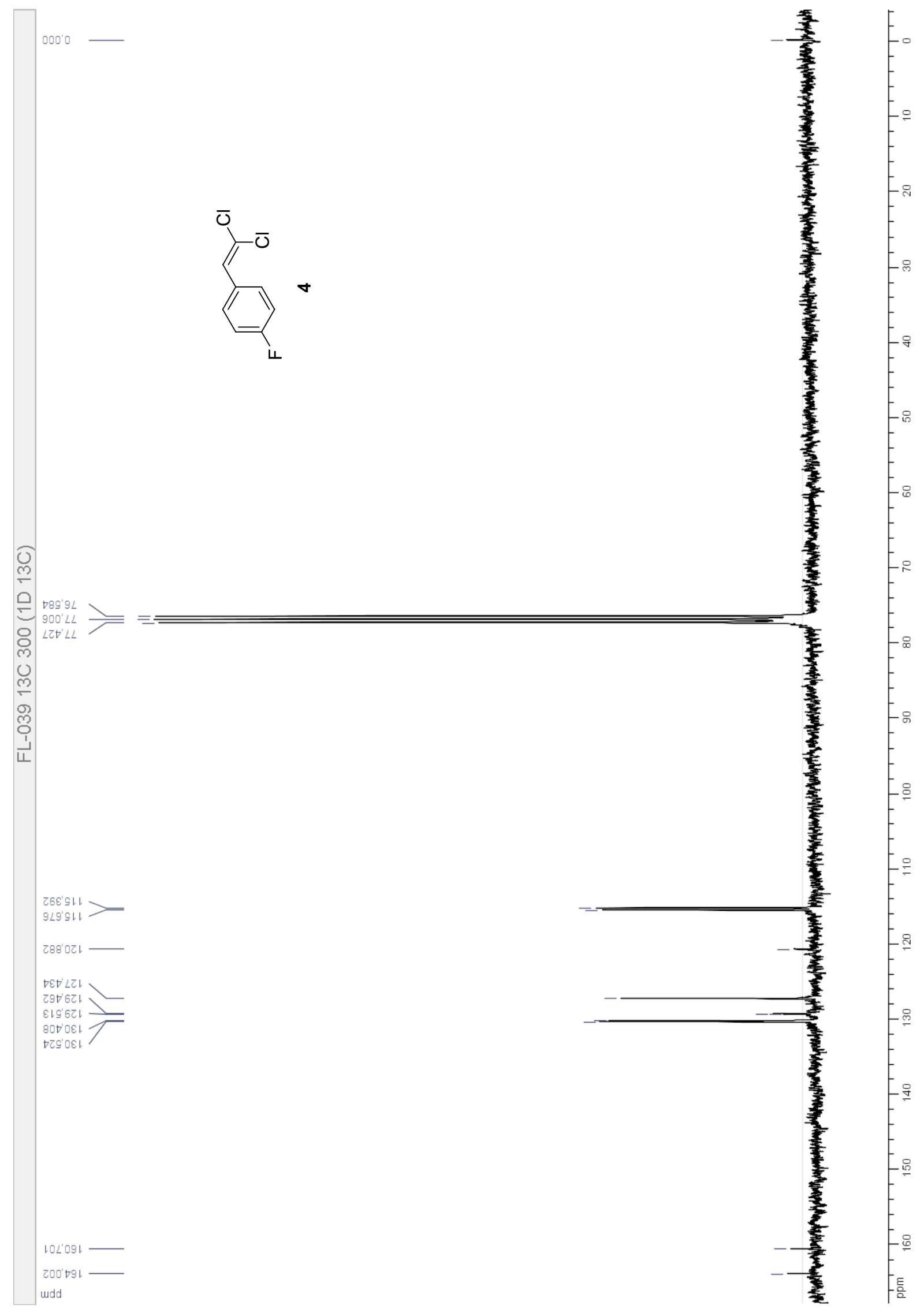




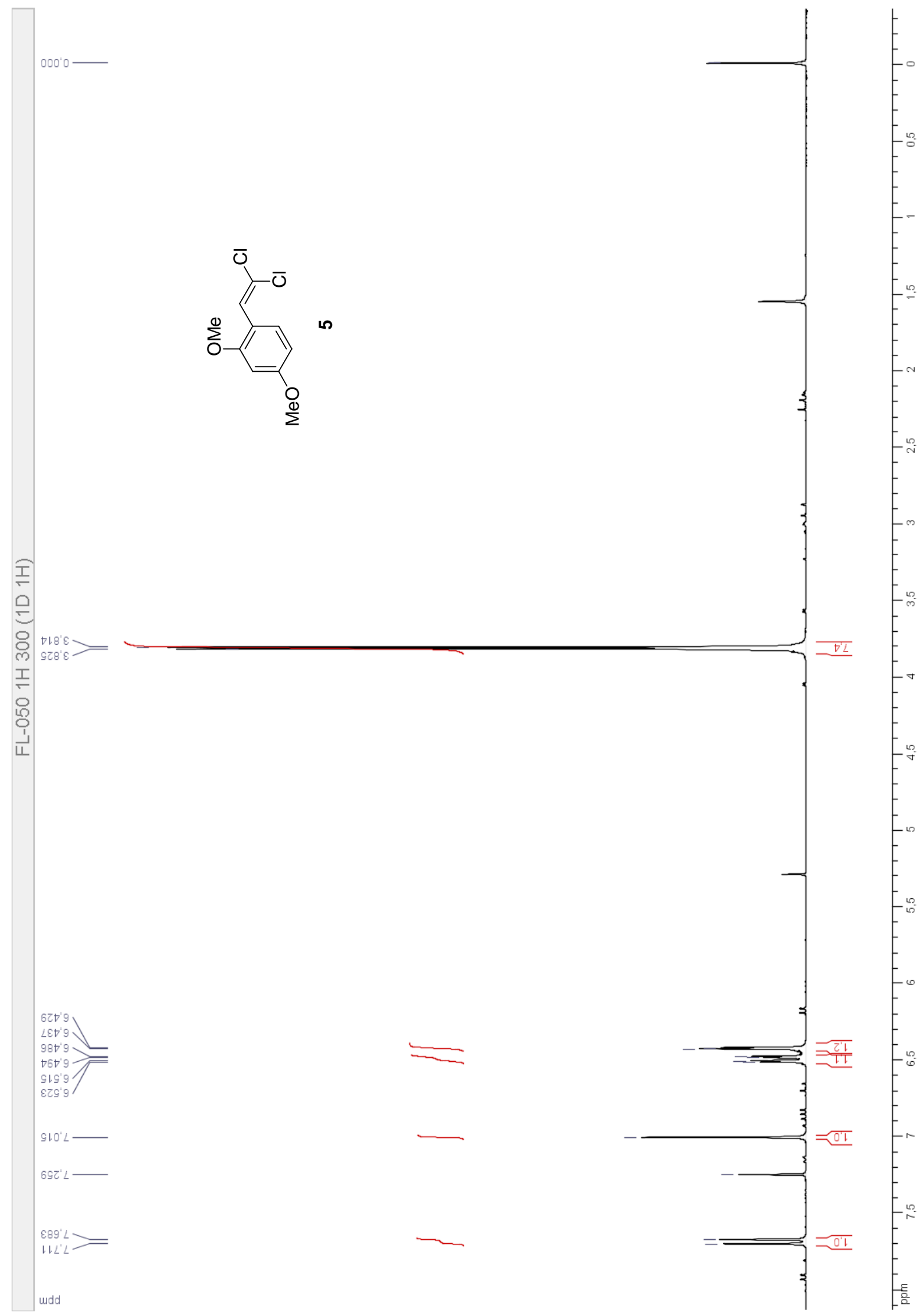



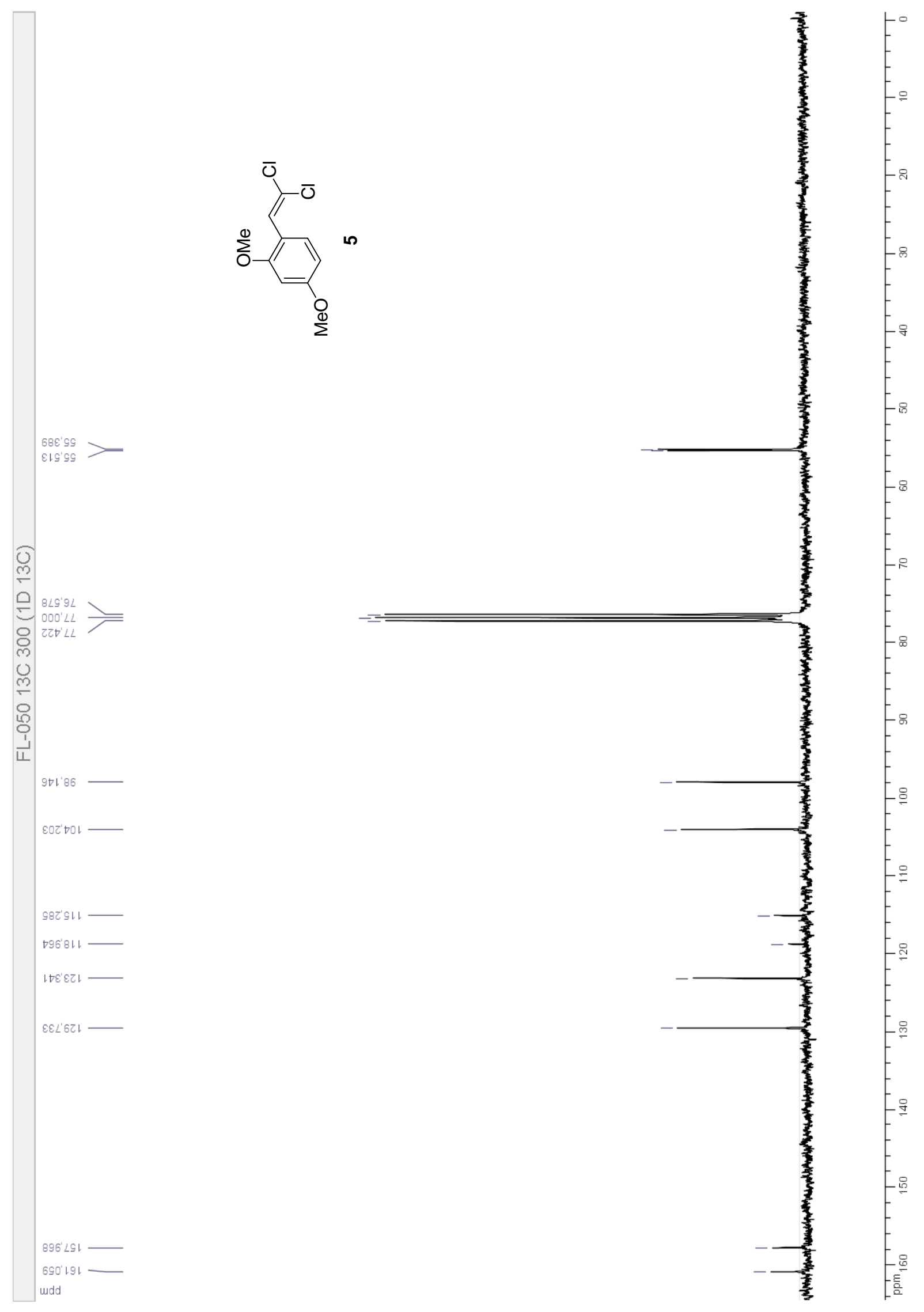


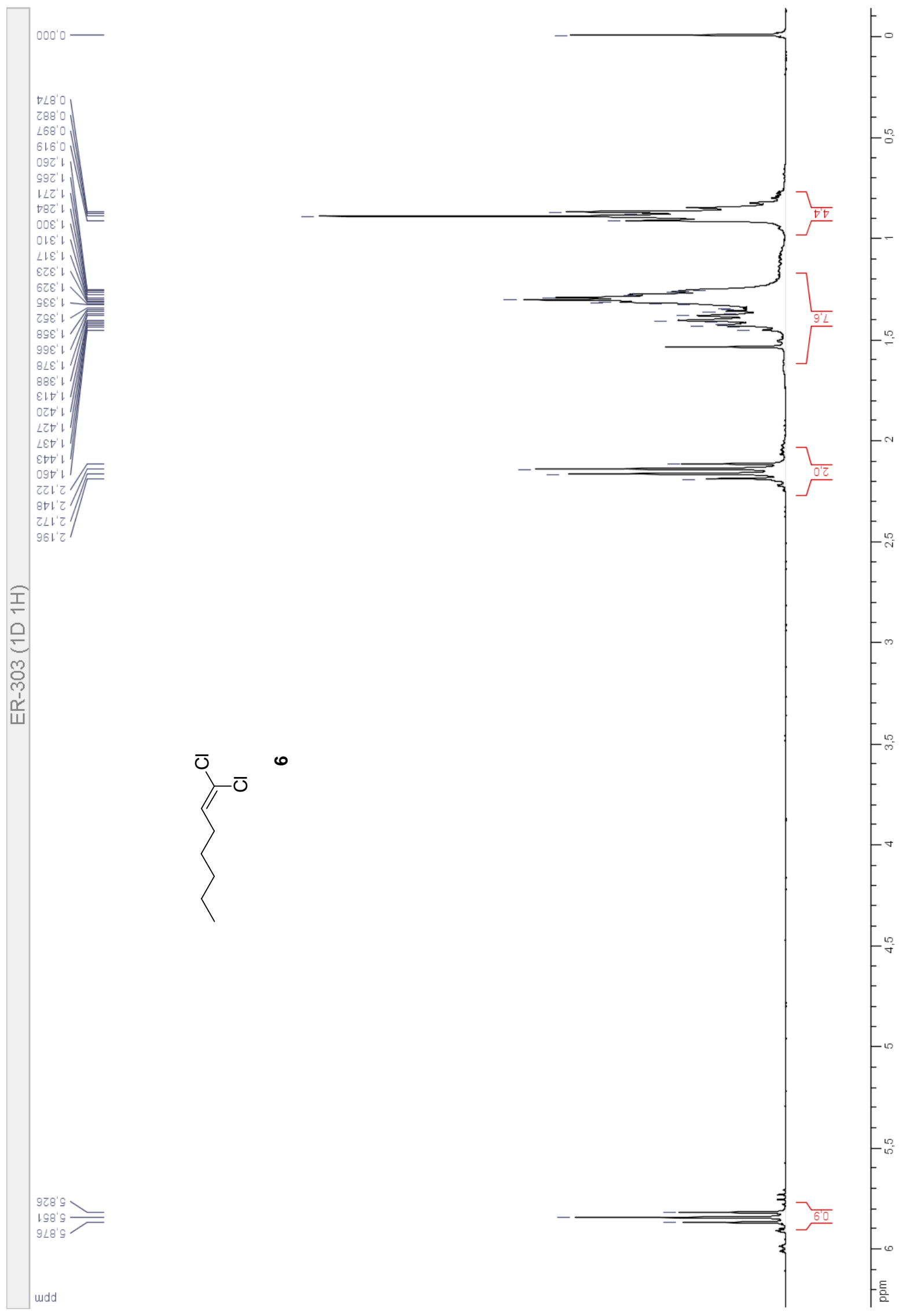



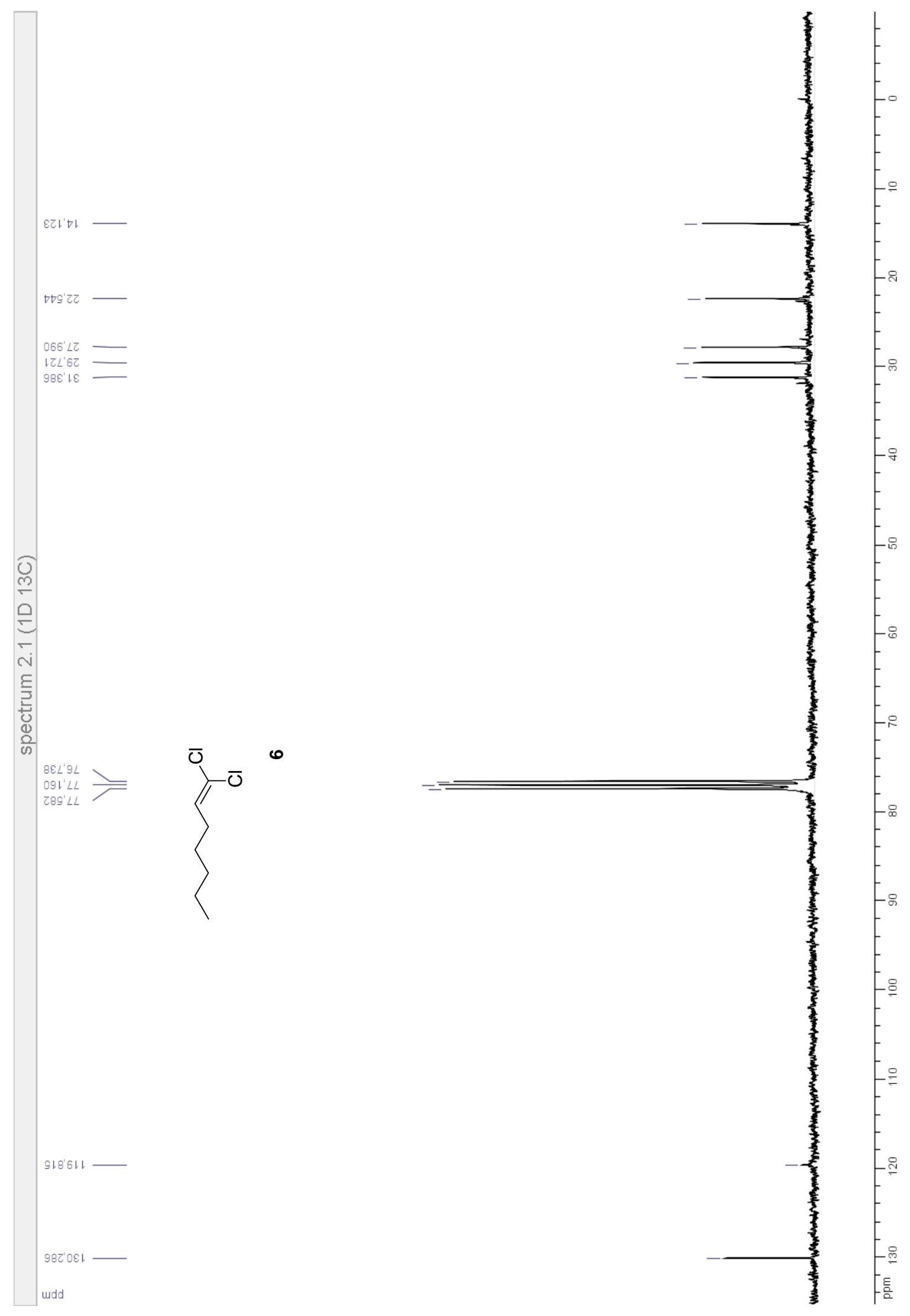


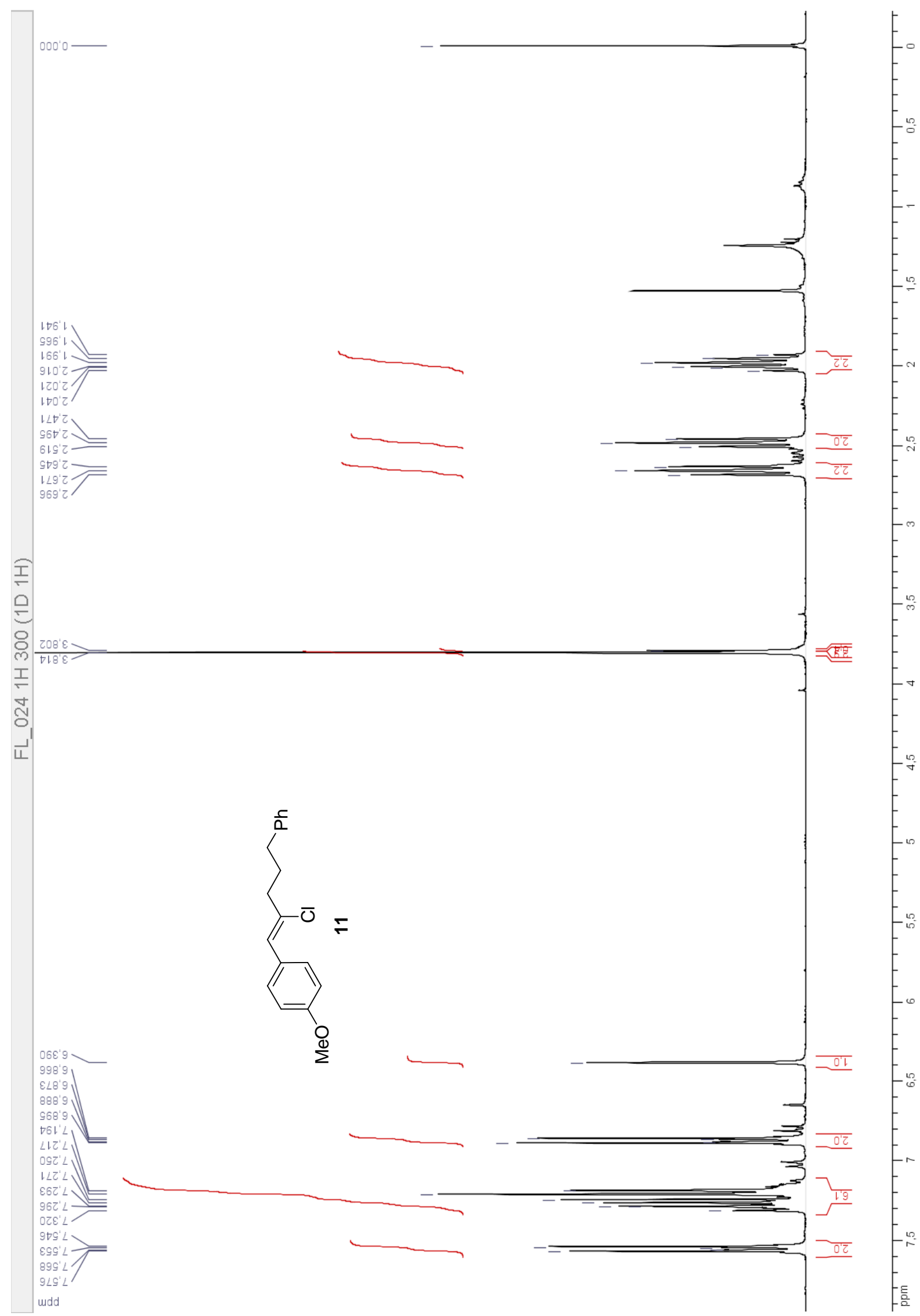




$$
11
$$




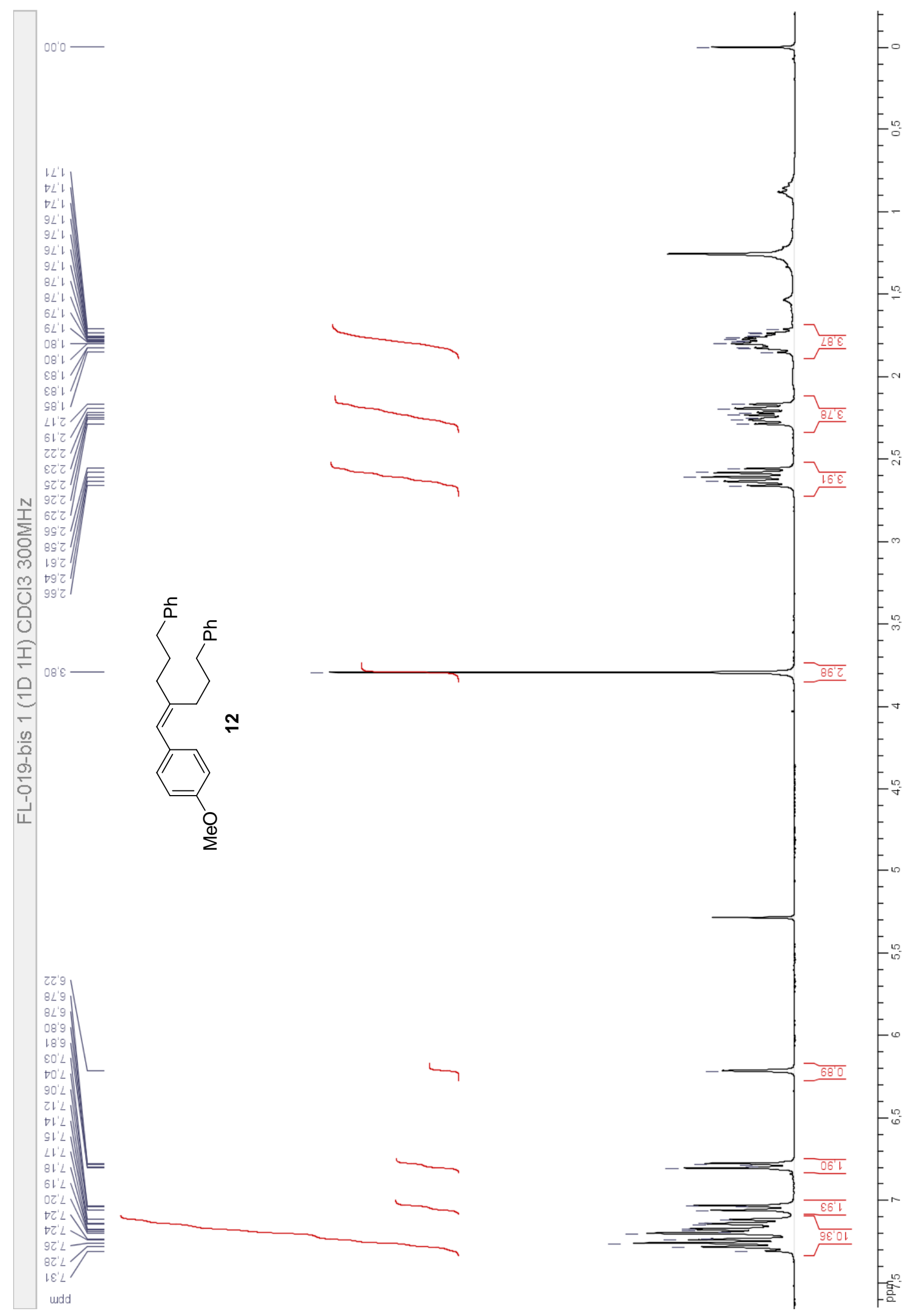




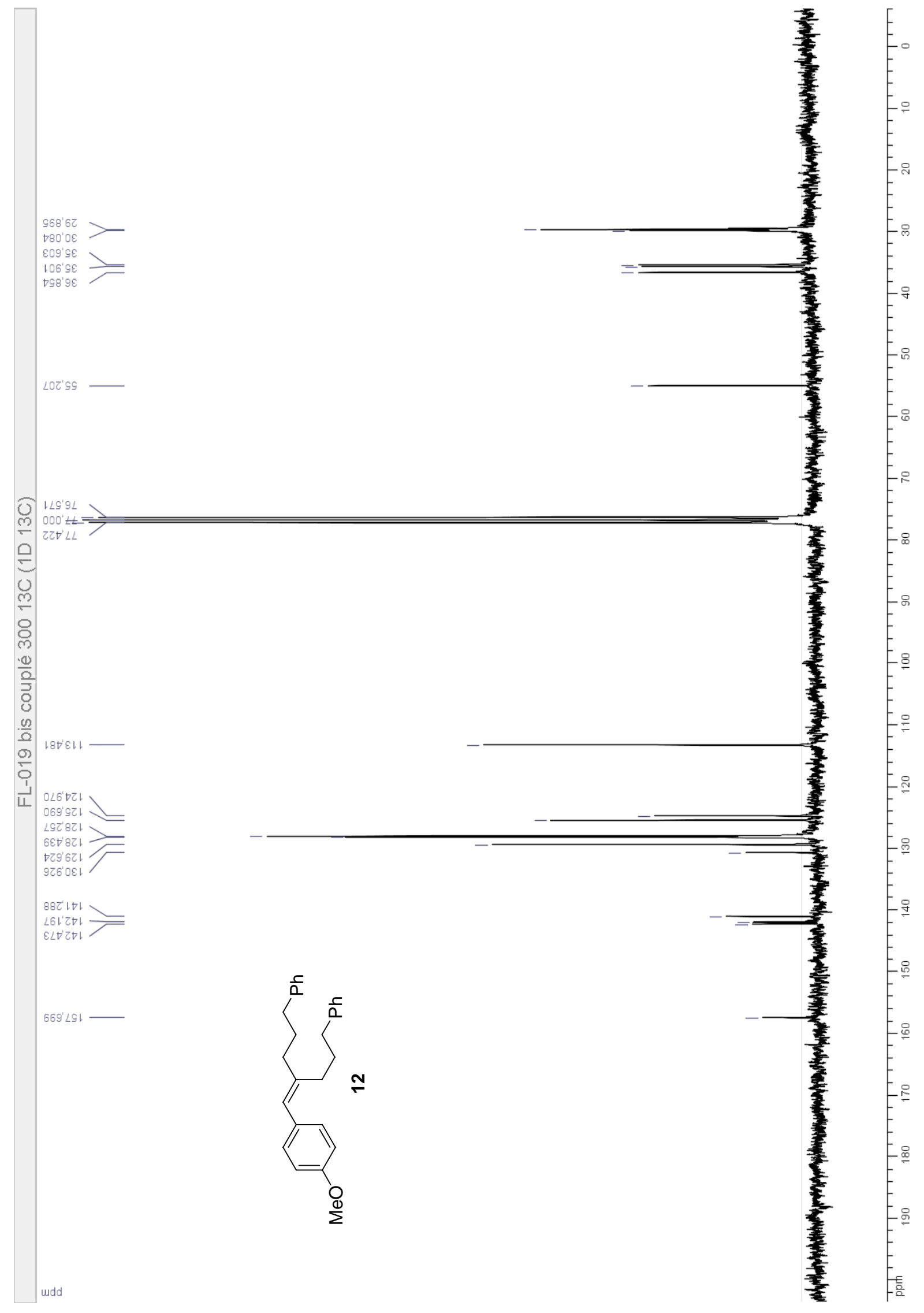




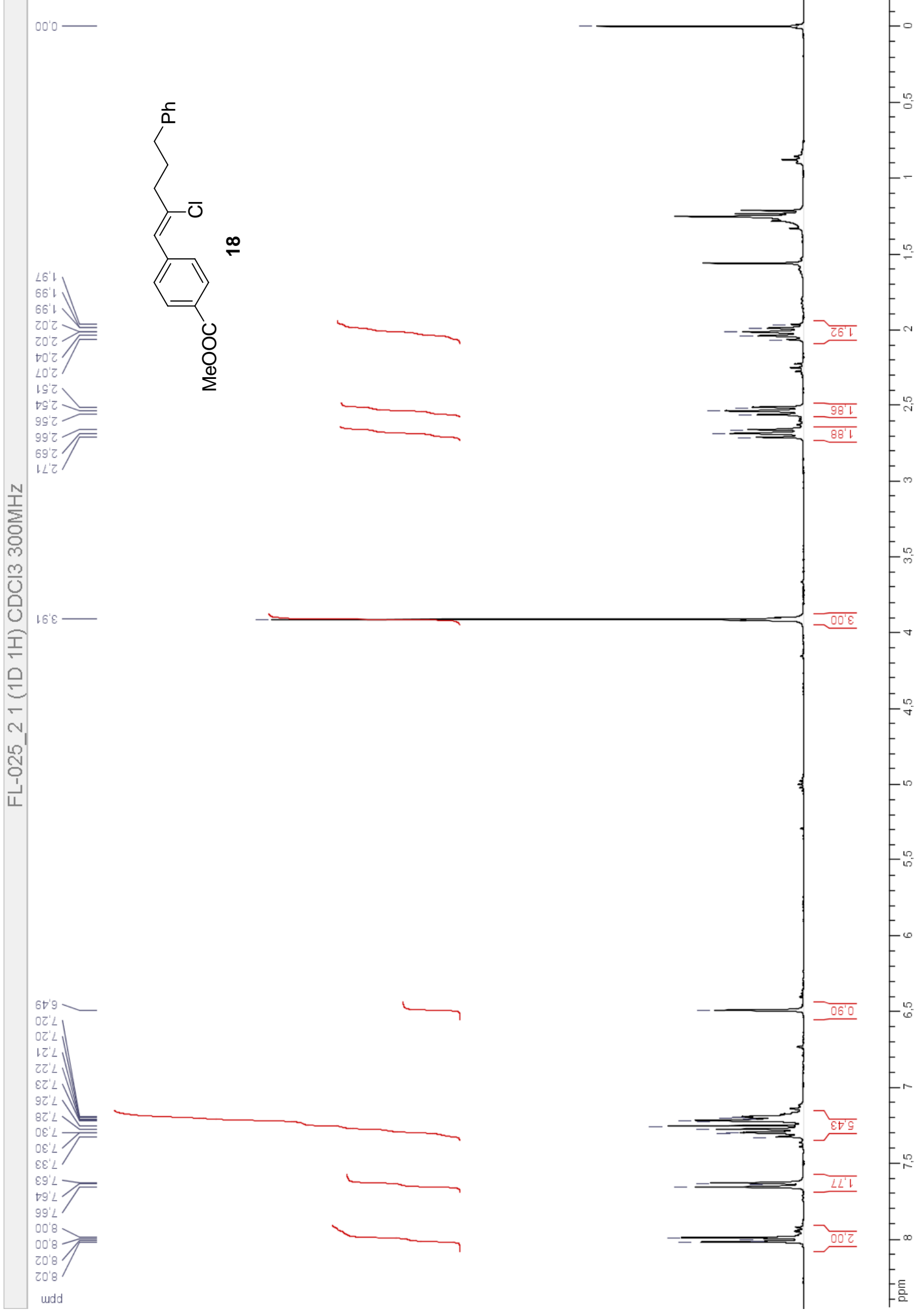




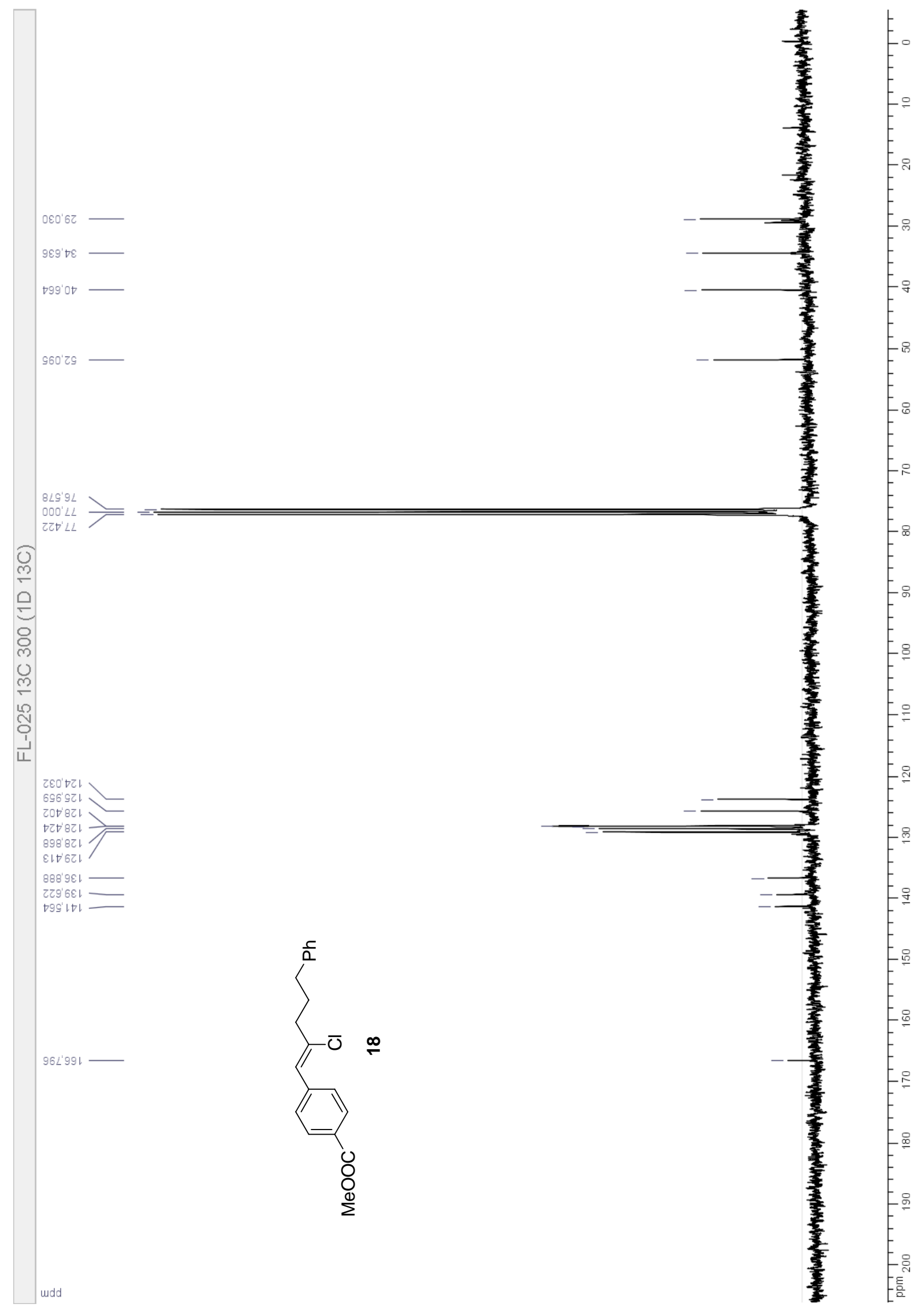




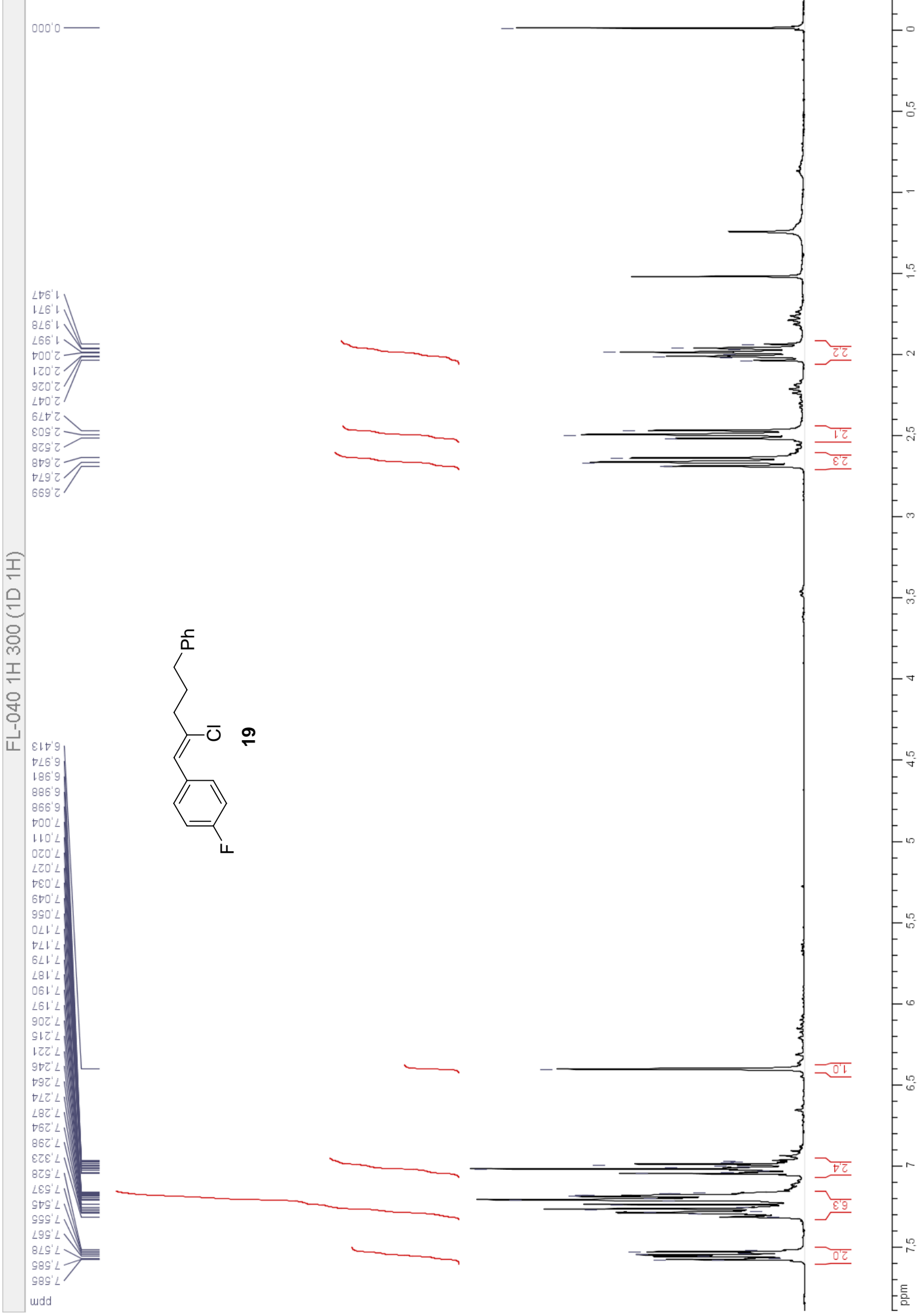



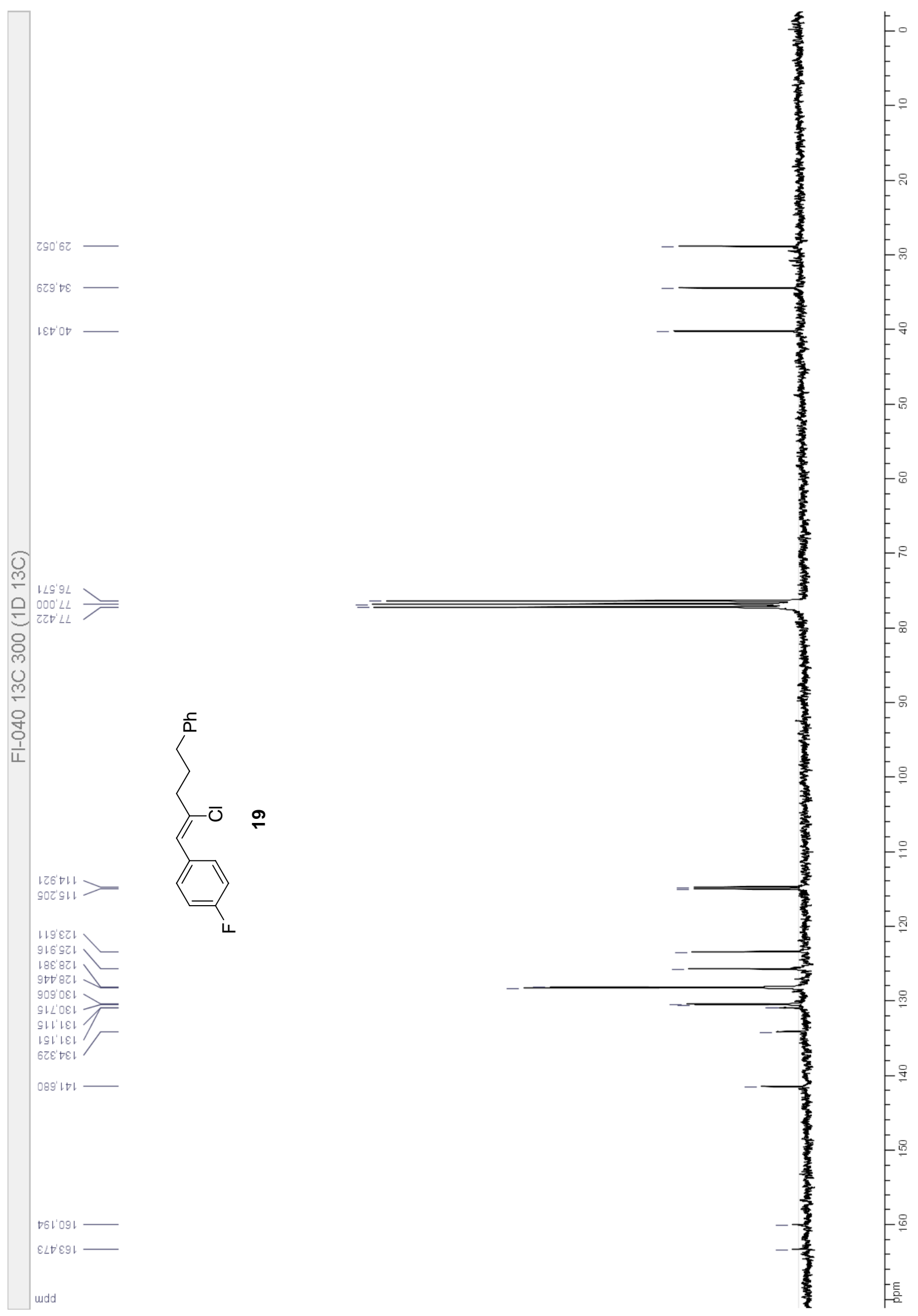

S34 


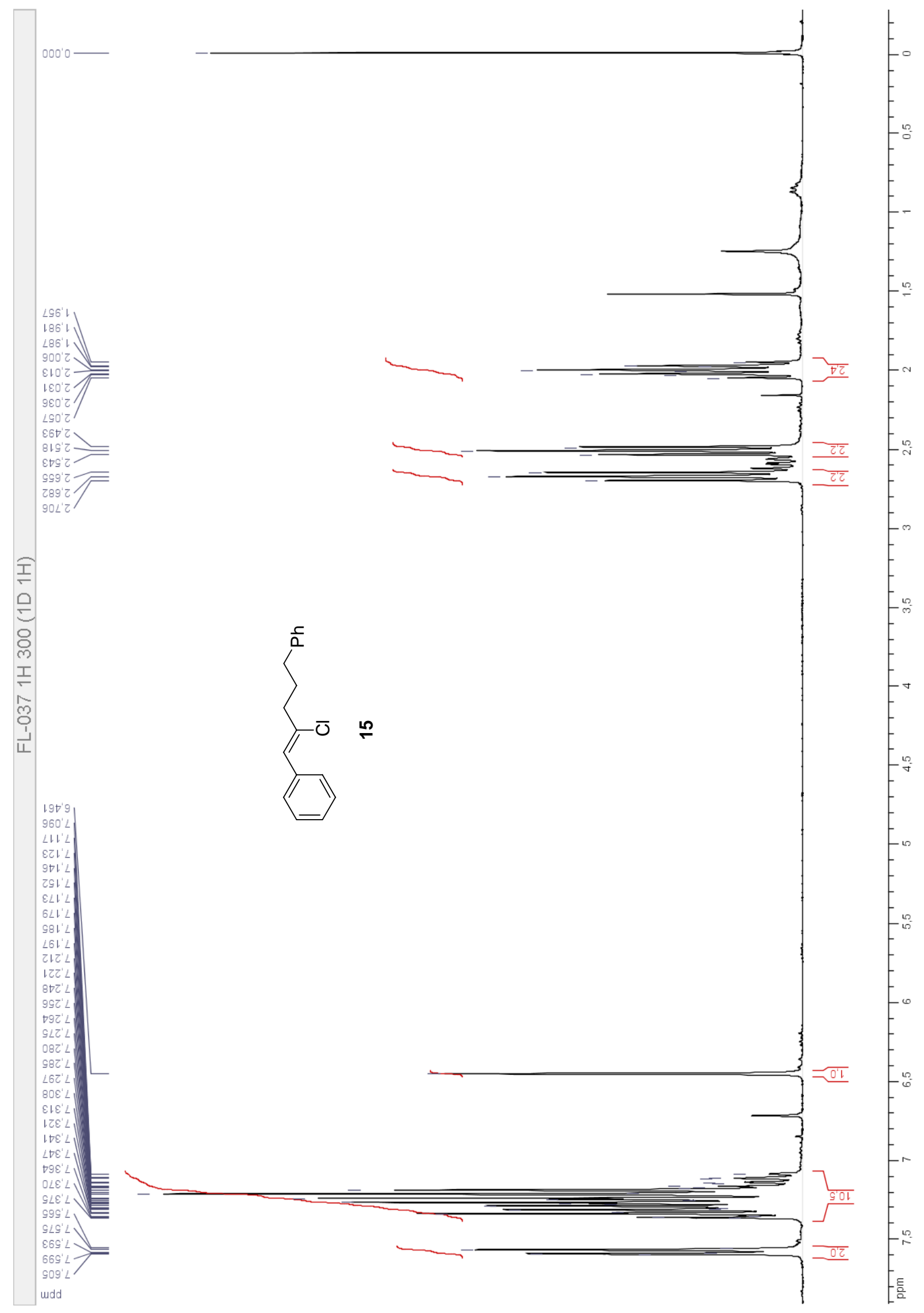



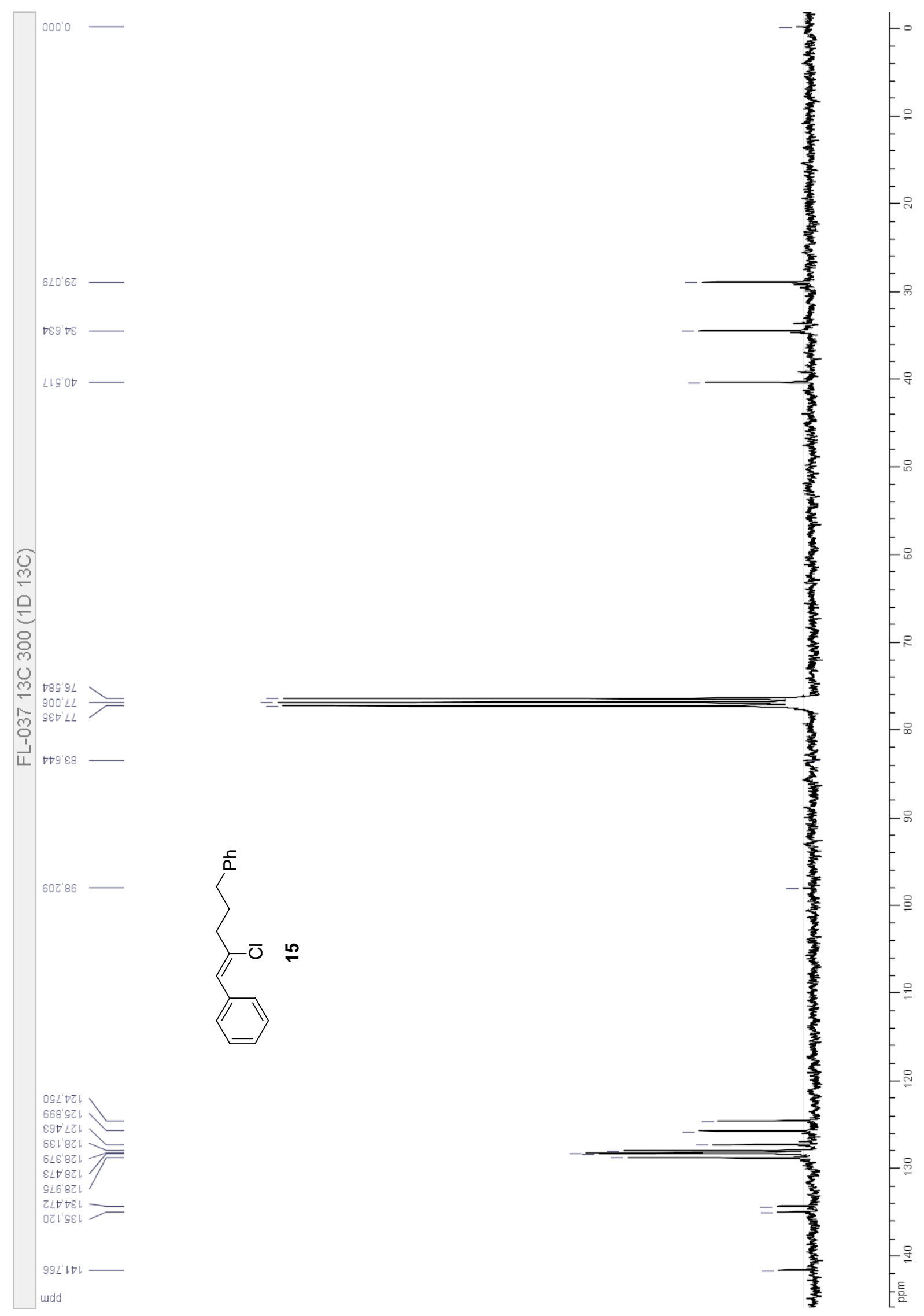


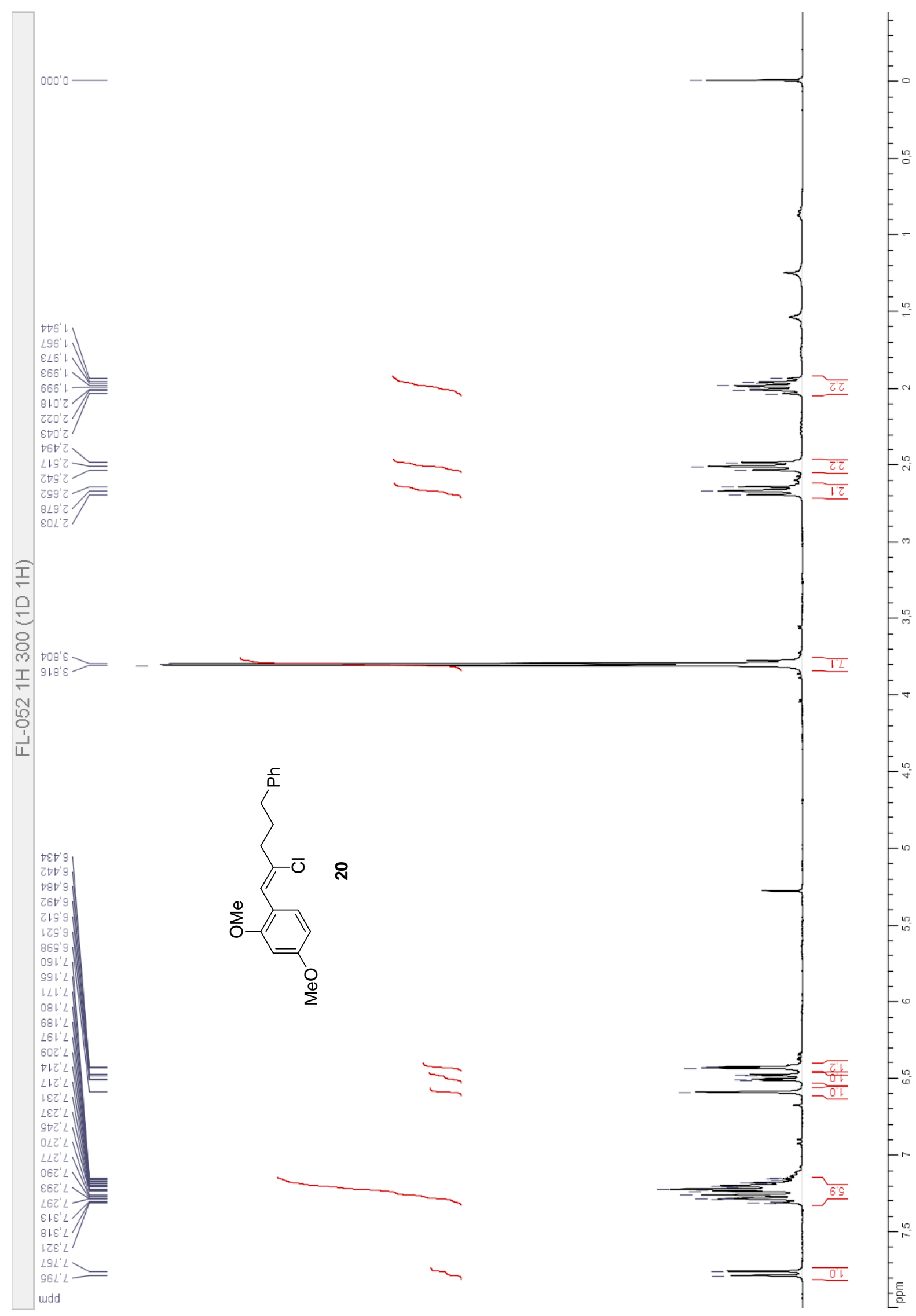




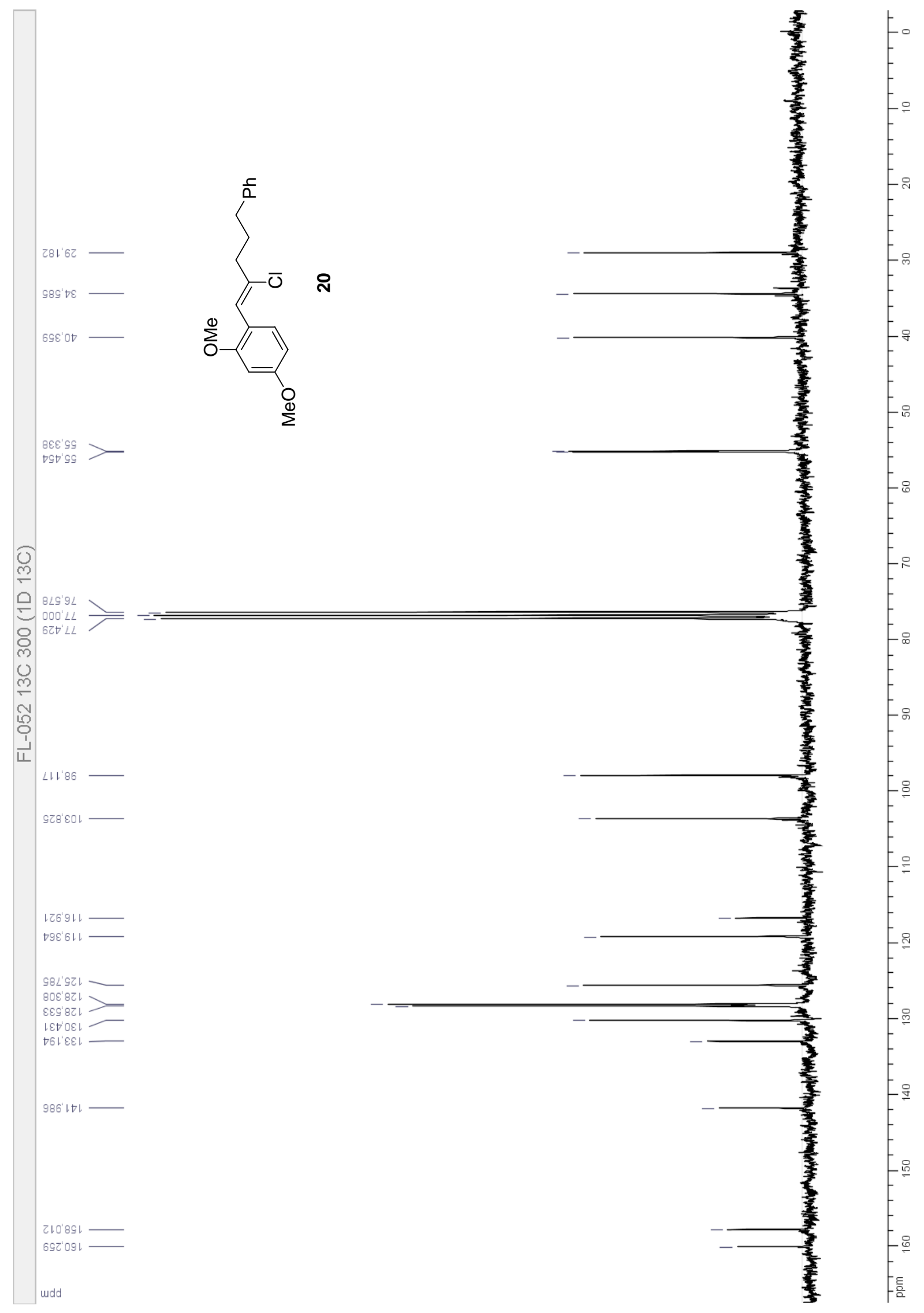




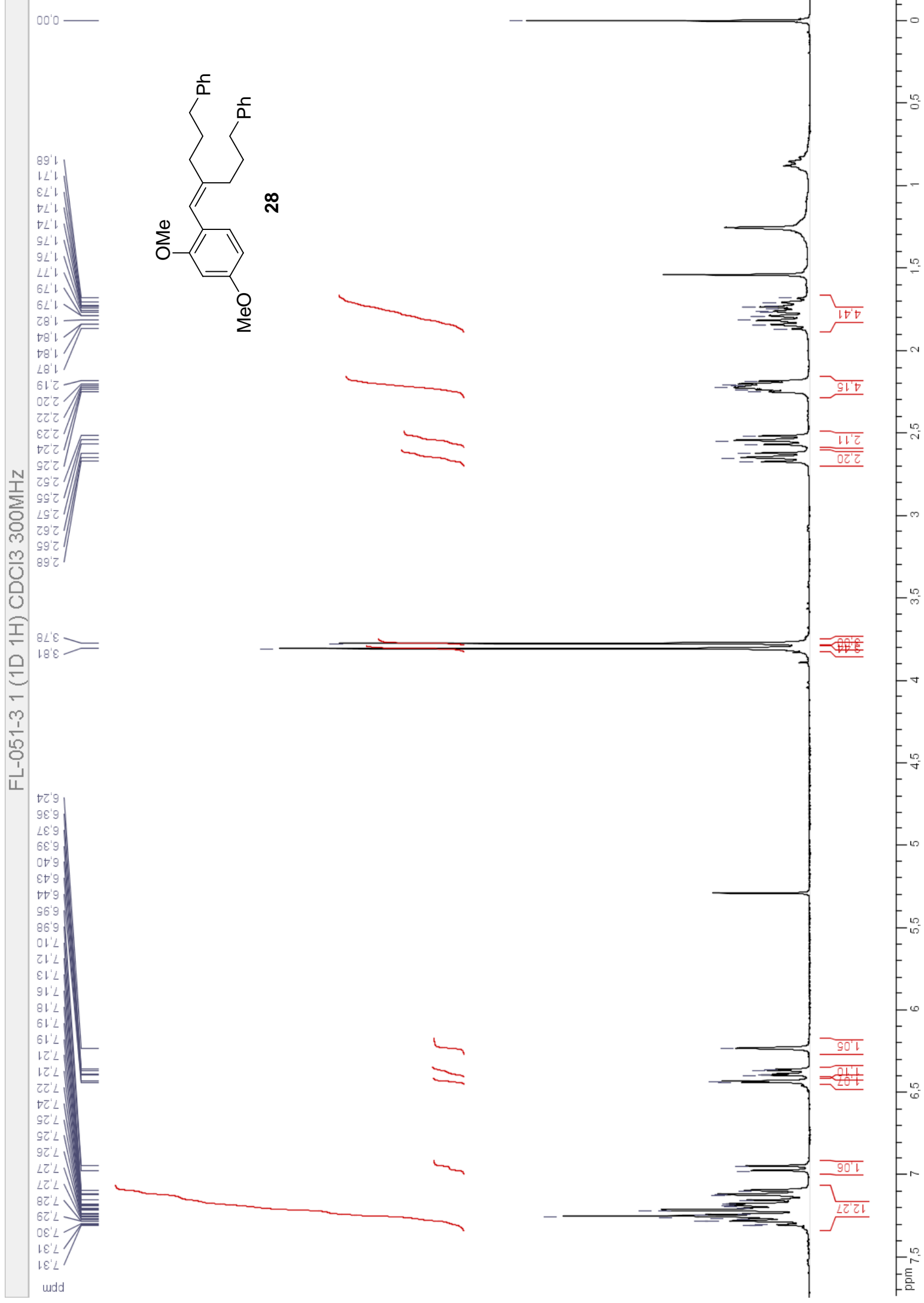




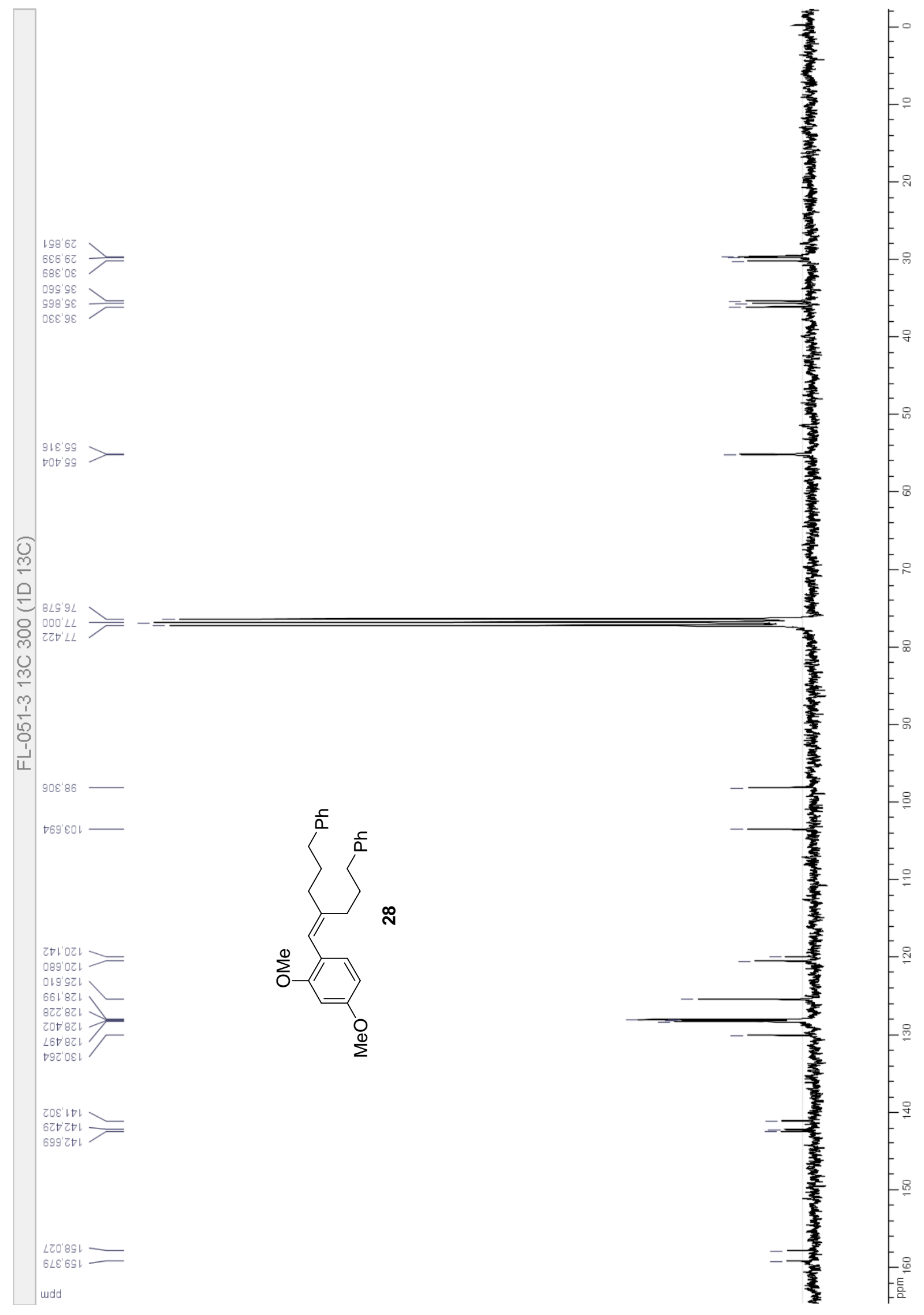




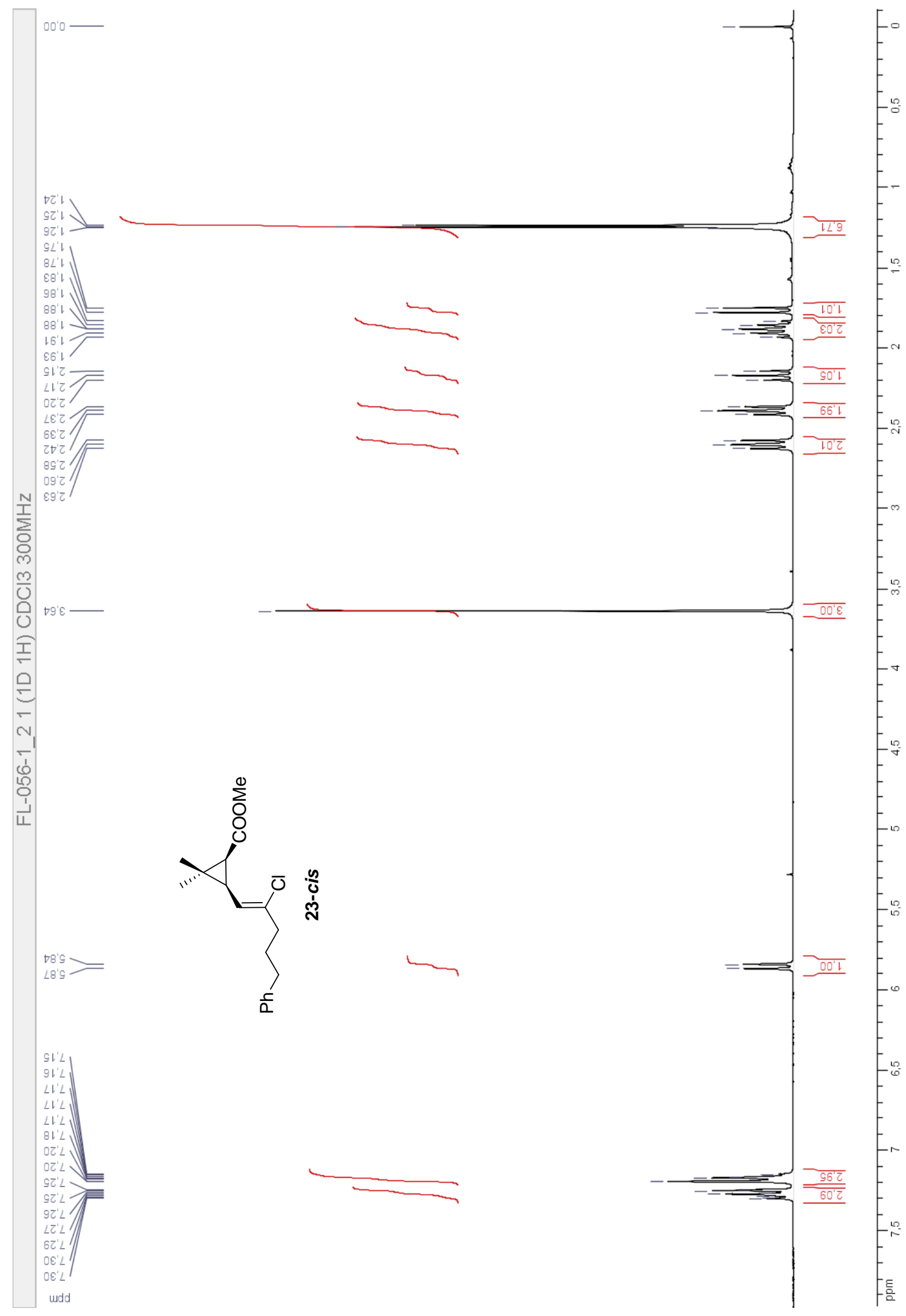



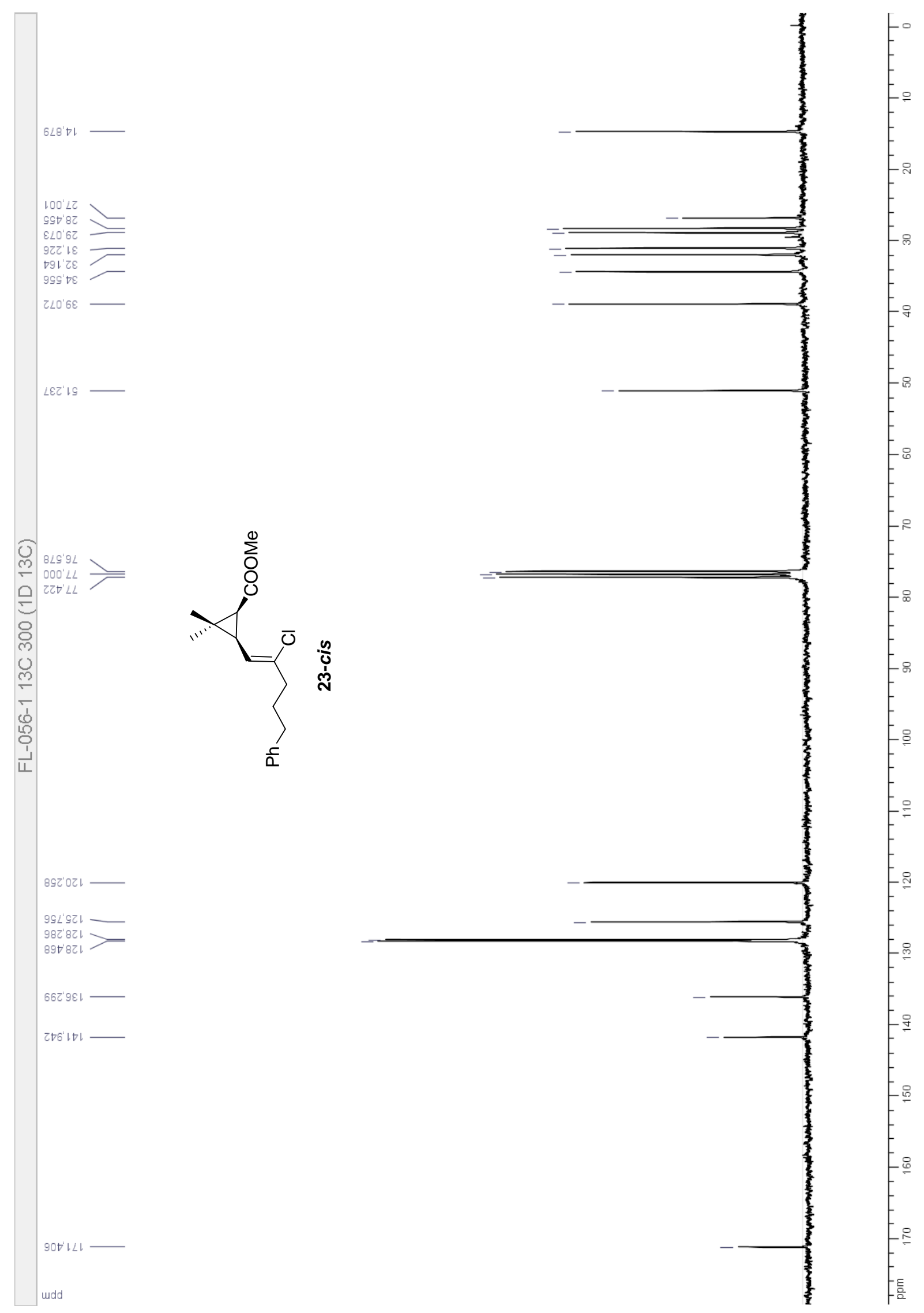


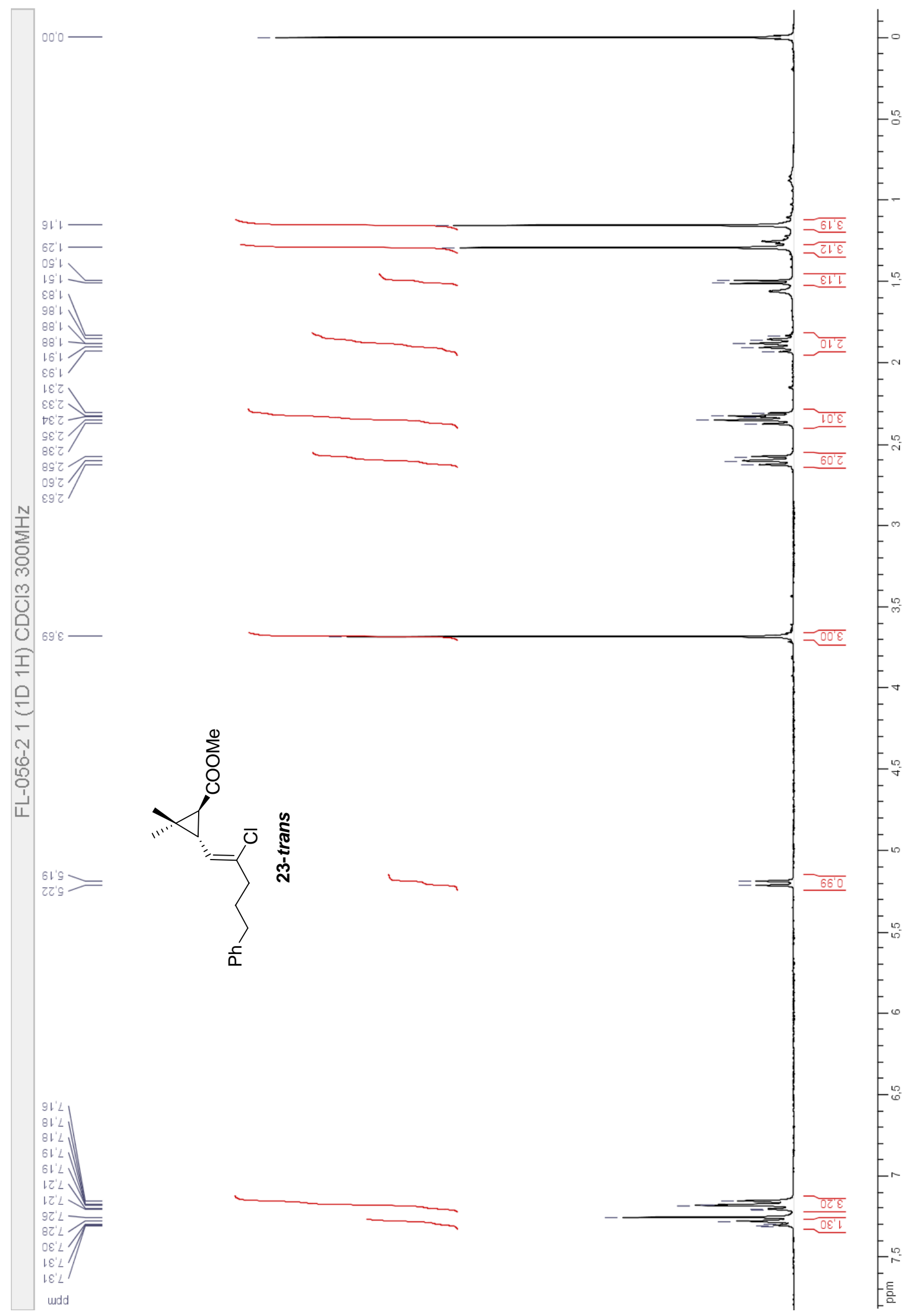



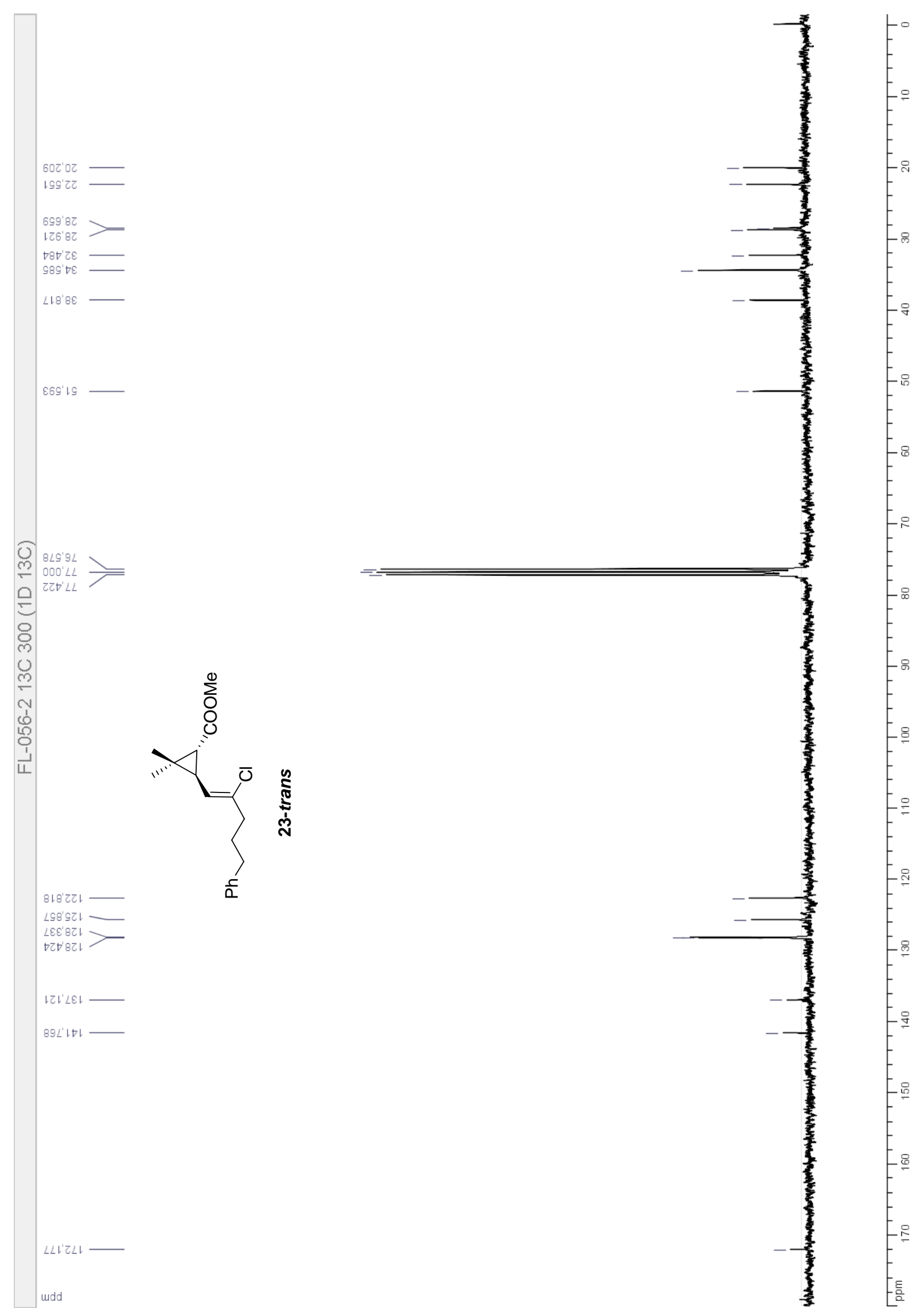


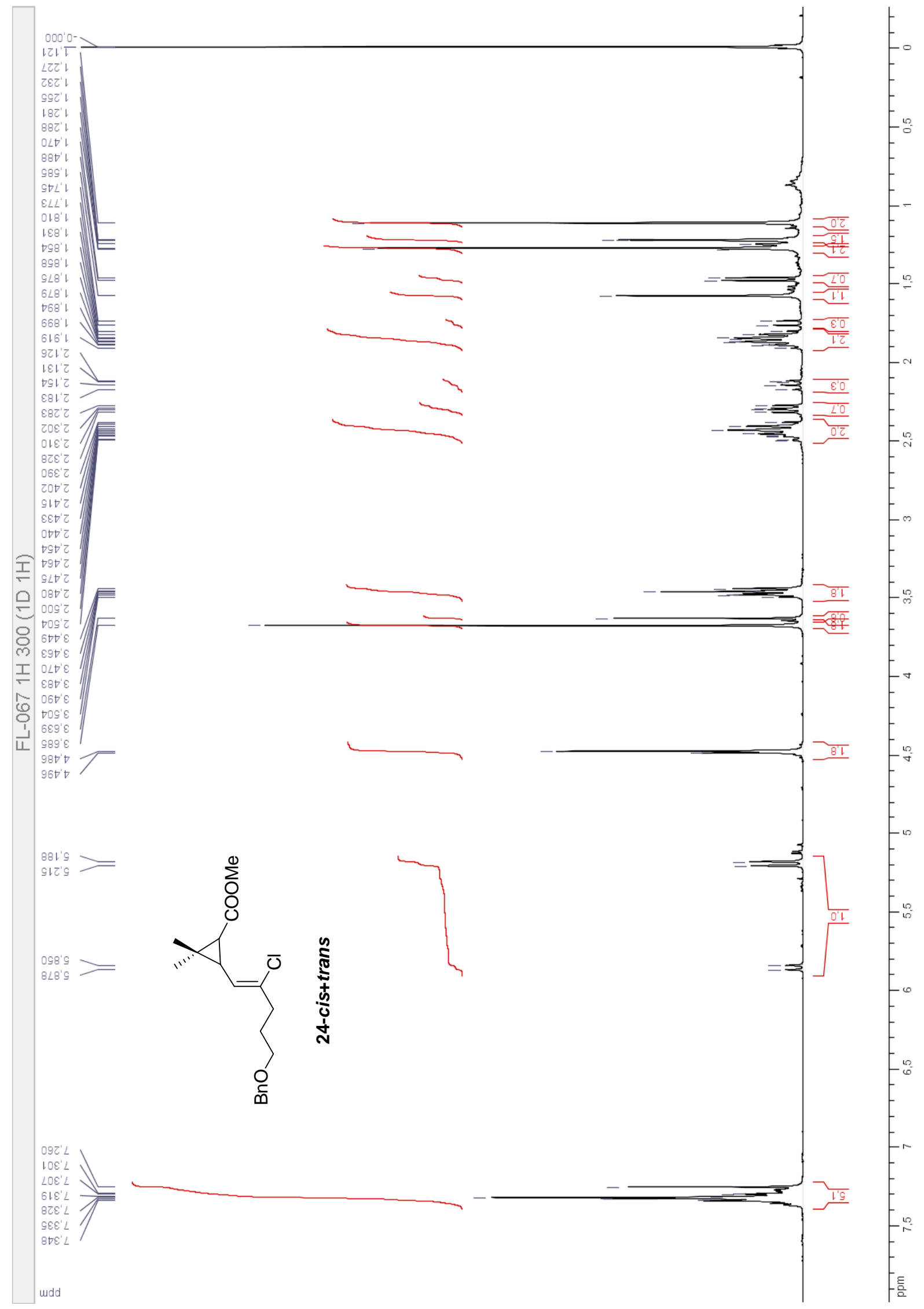



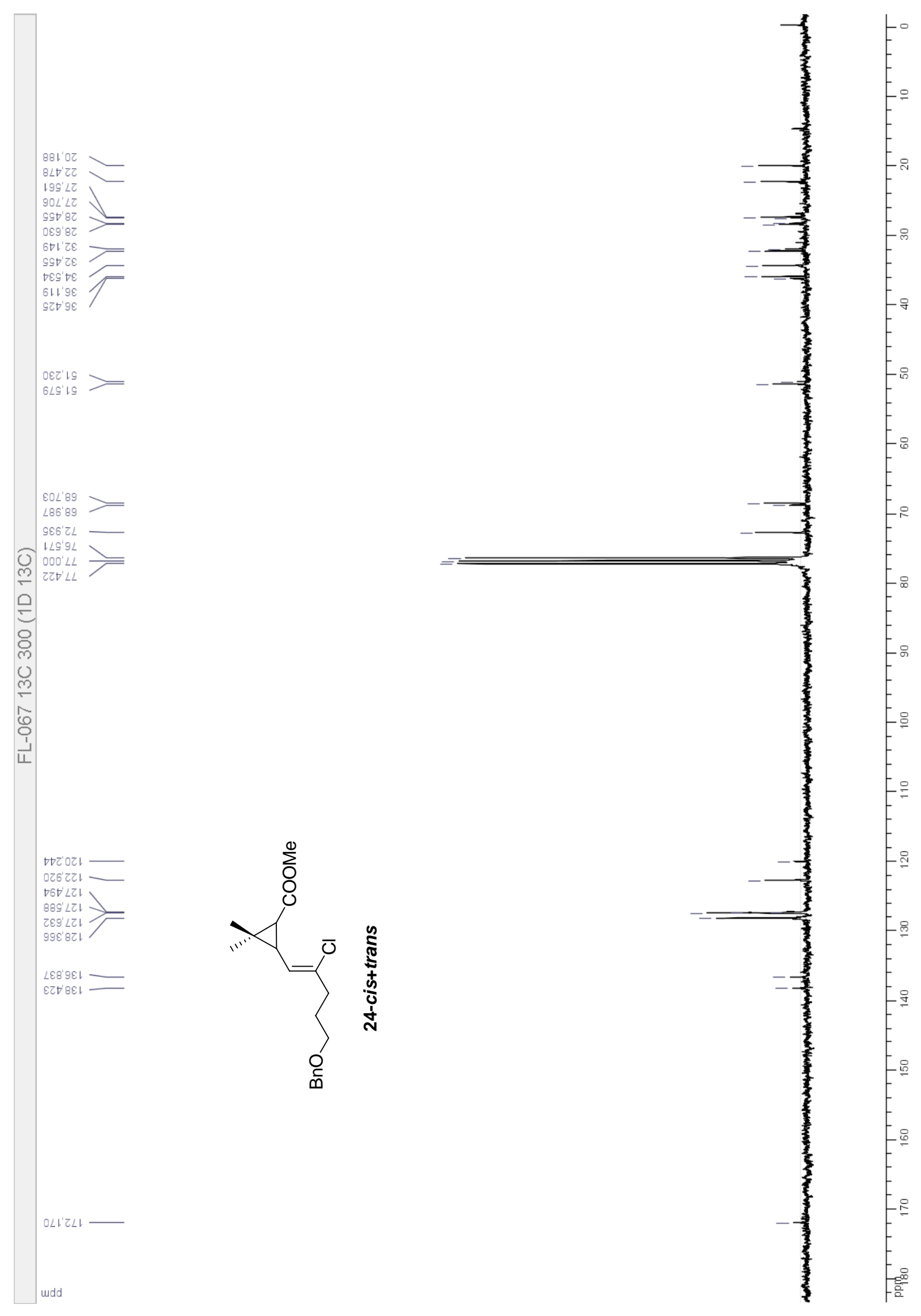


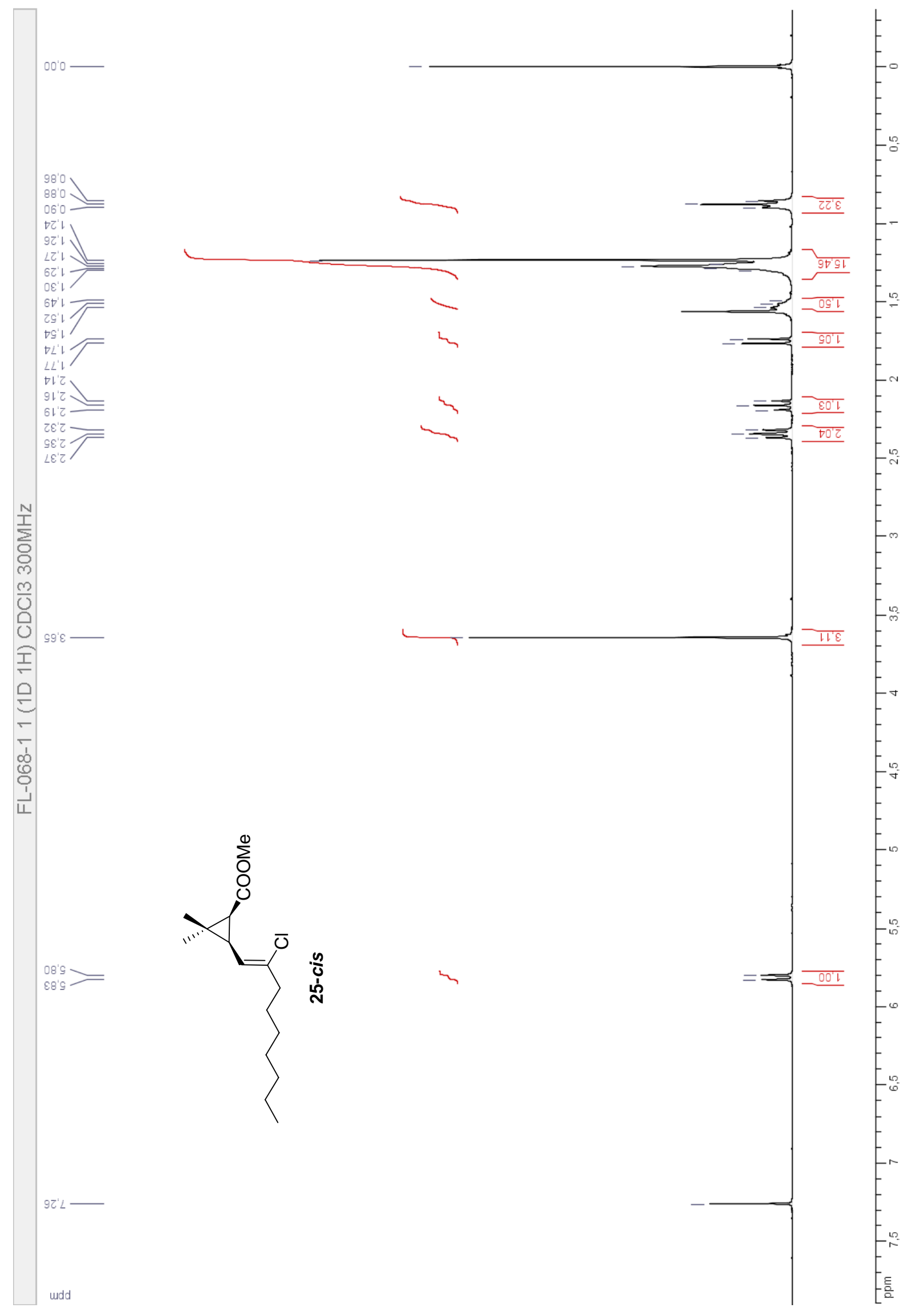




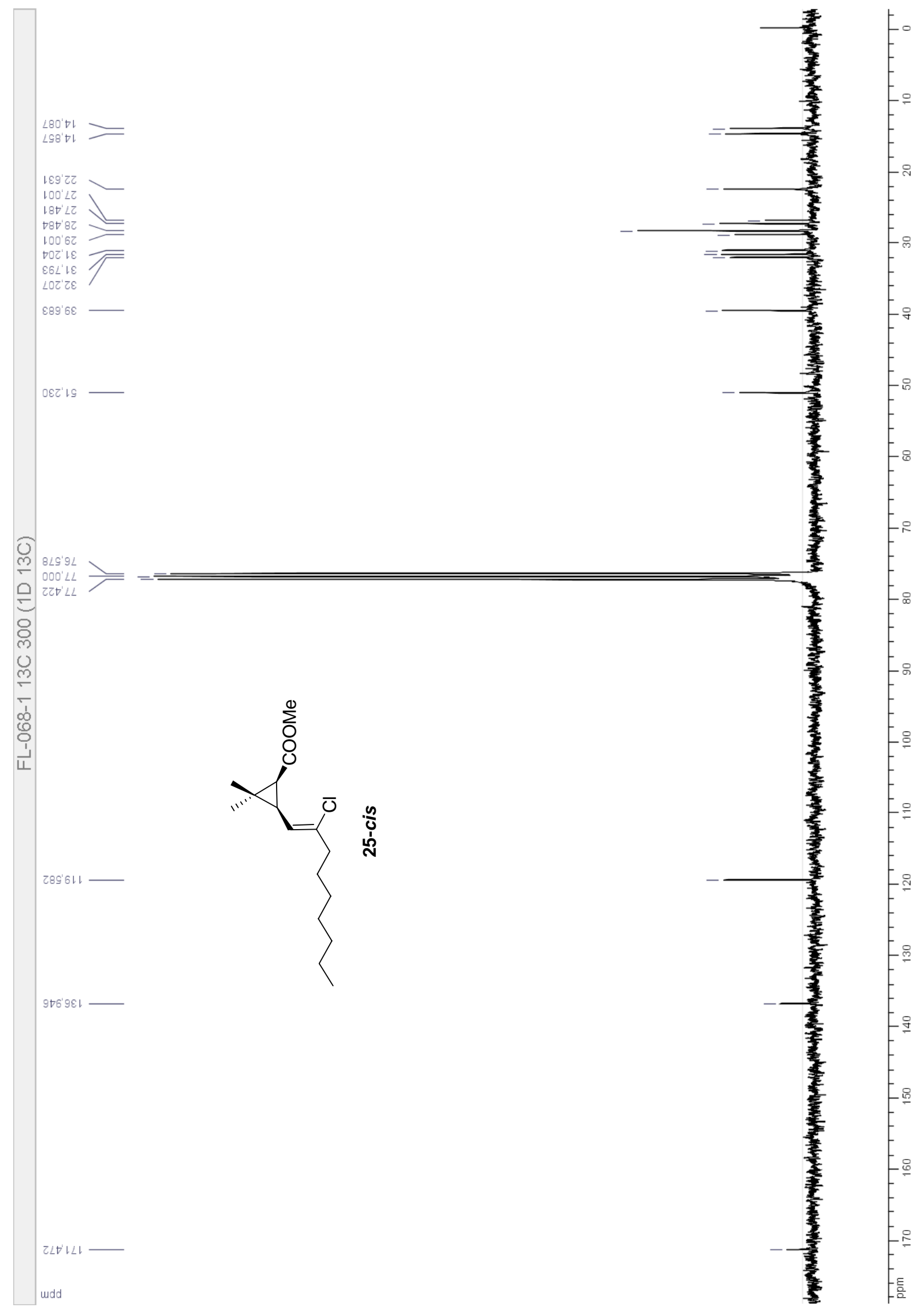




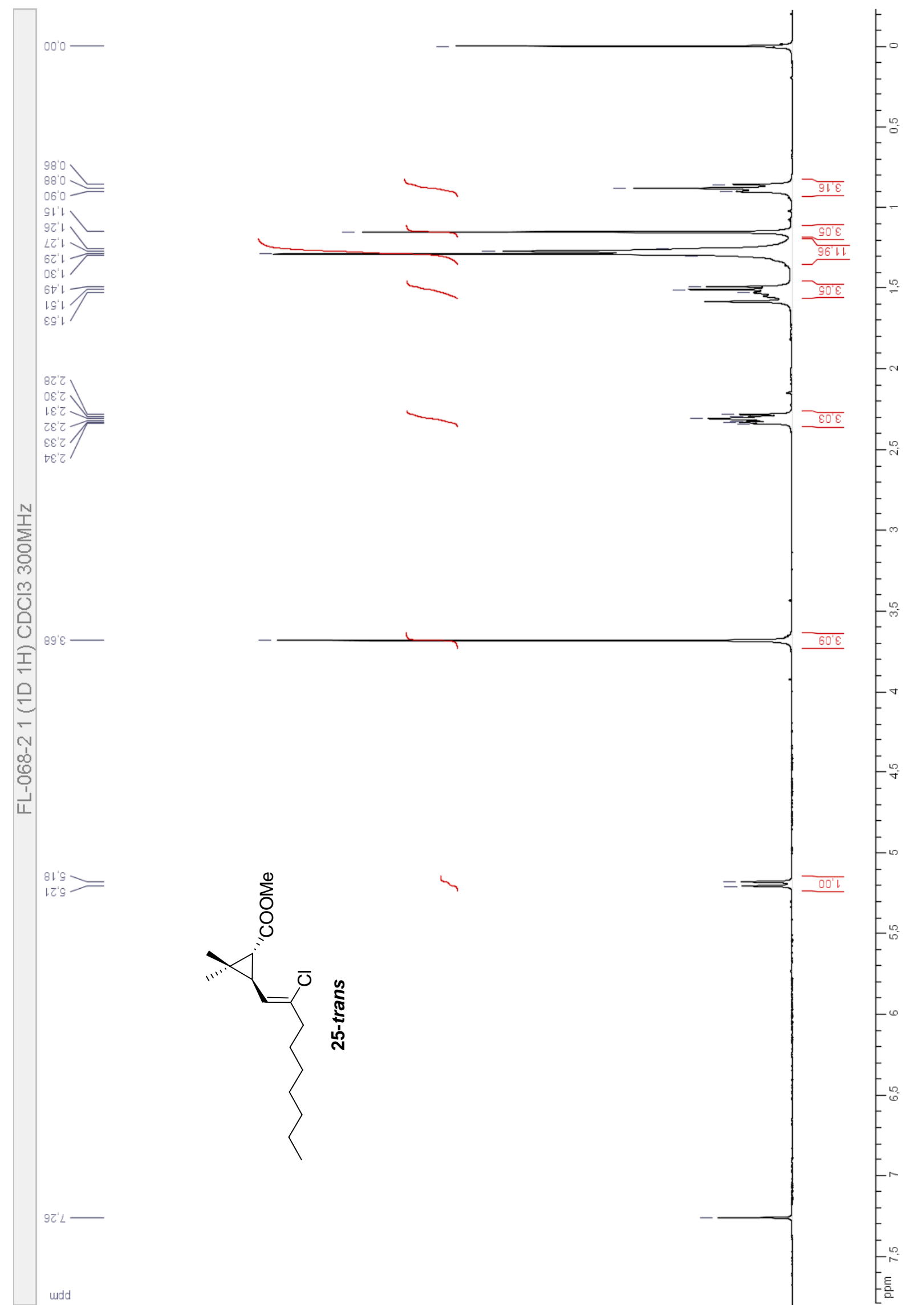



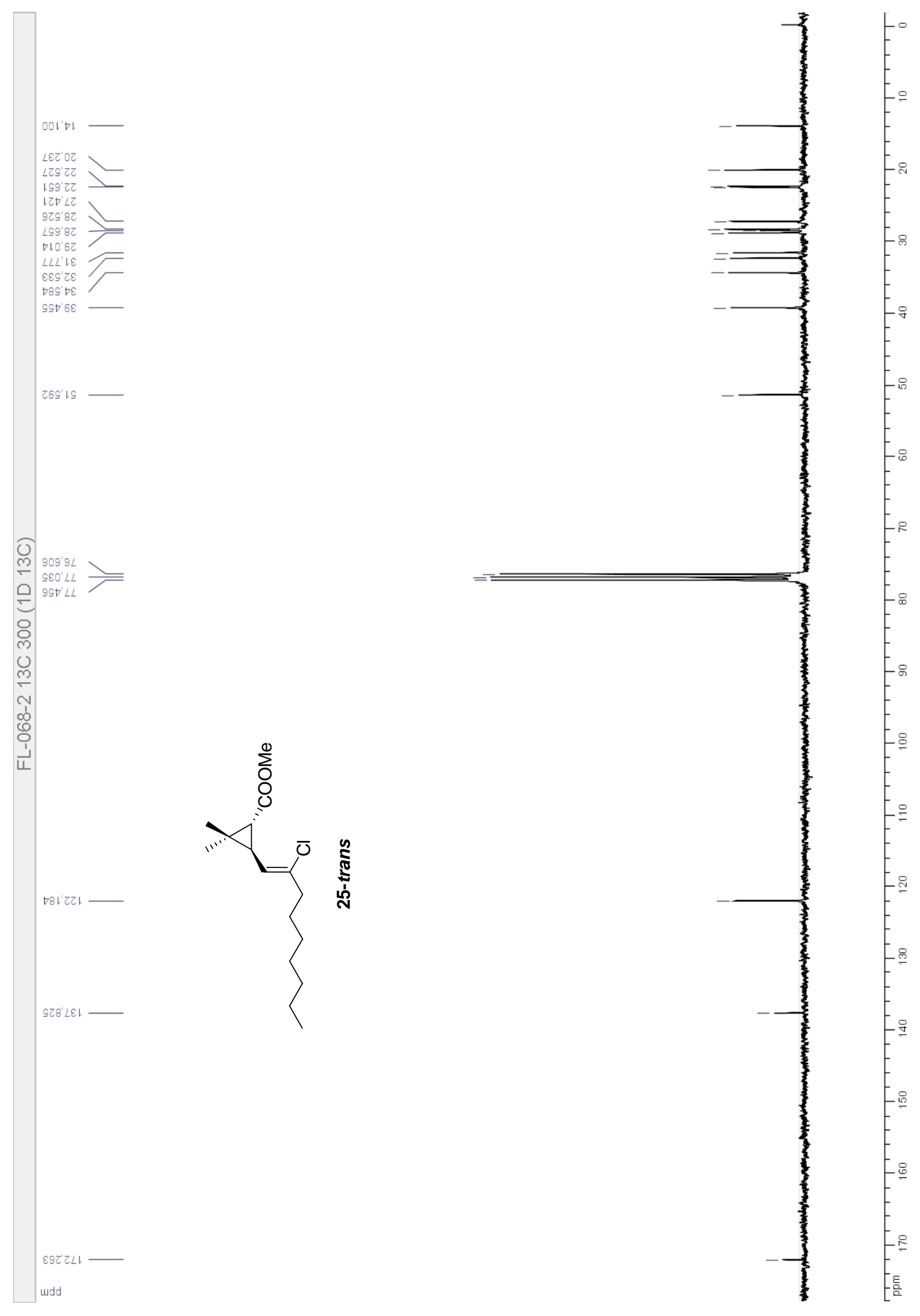


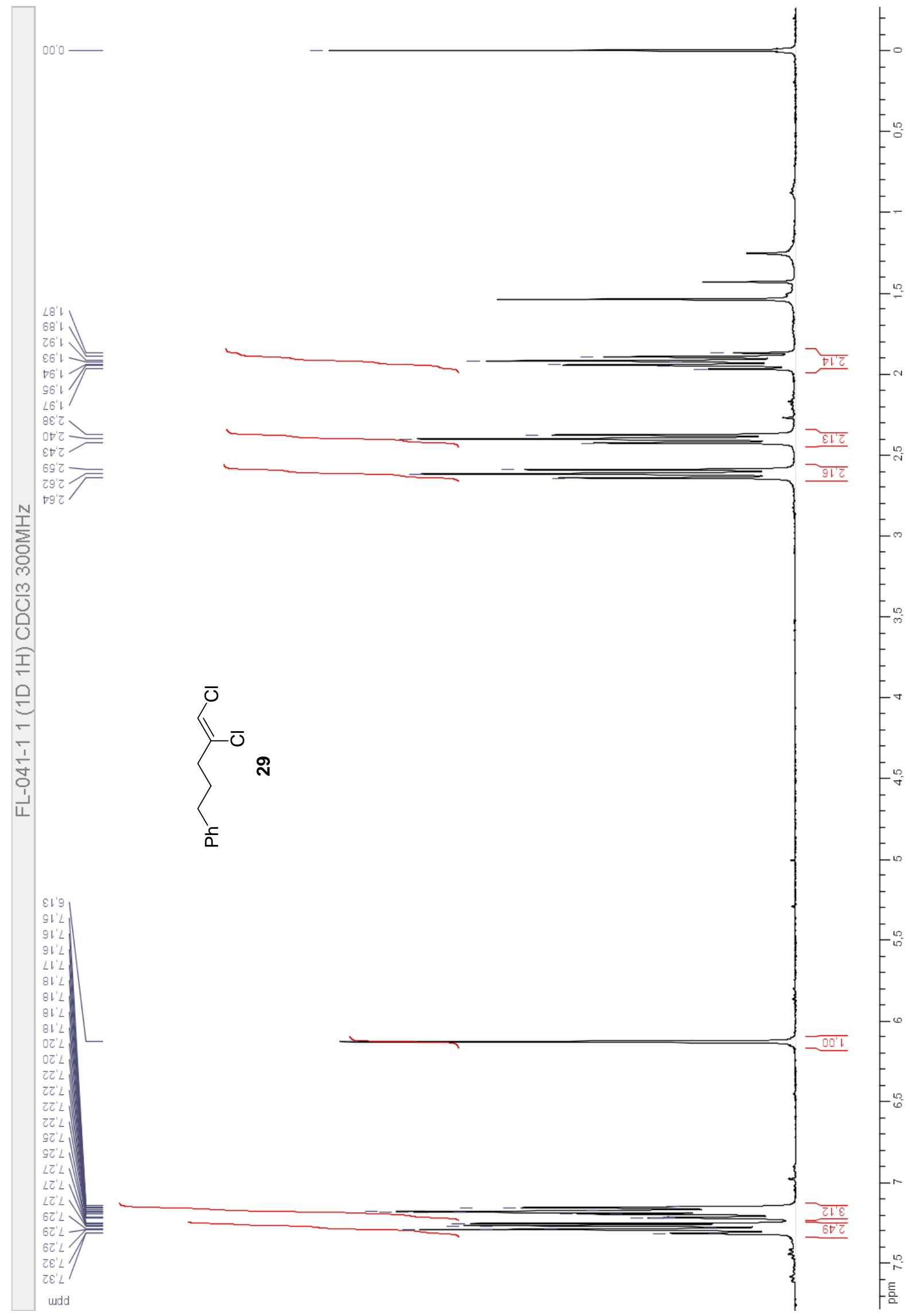




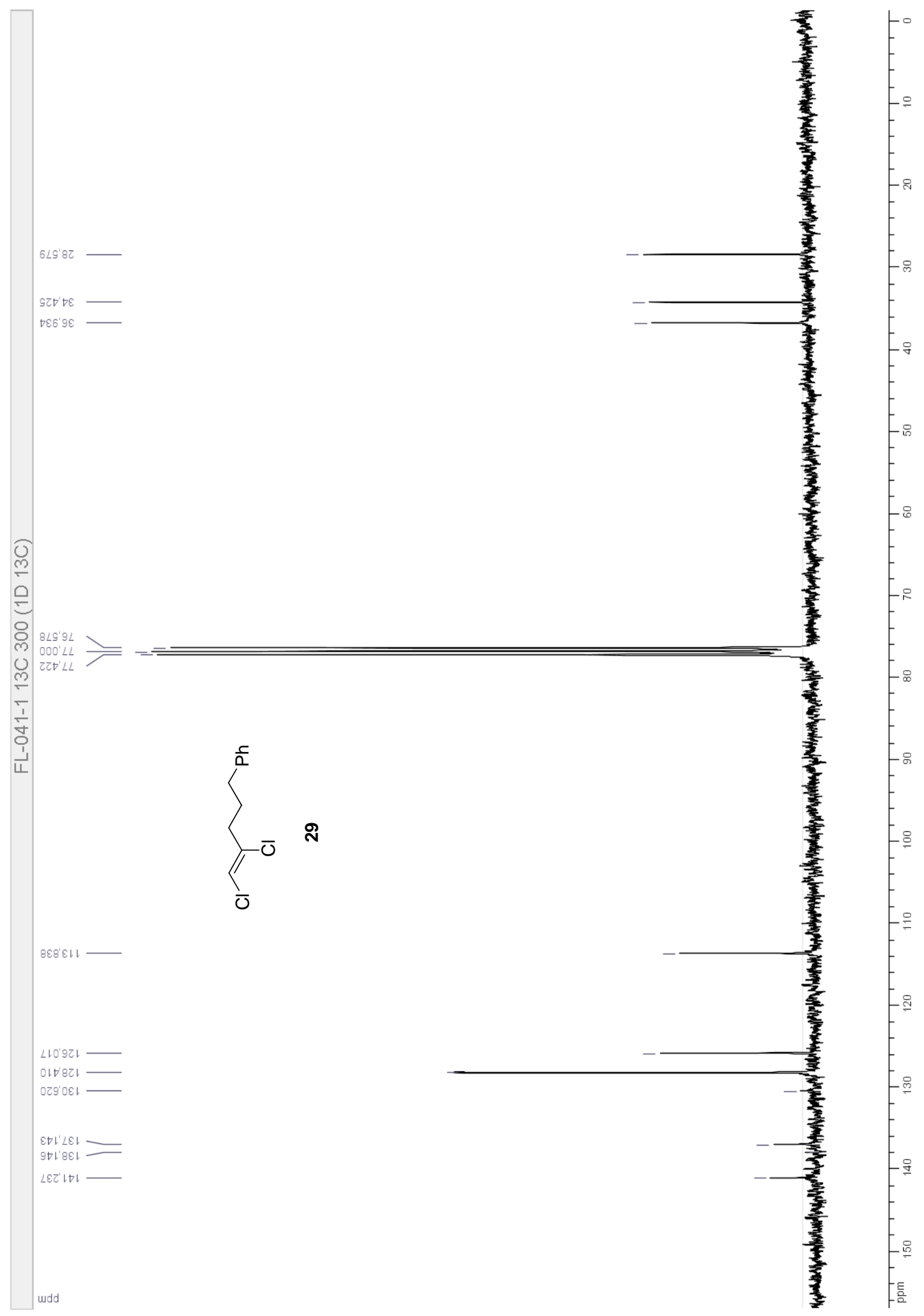

S52 


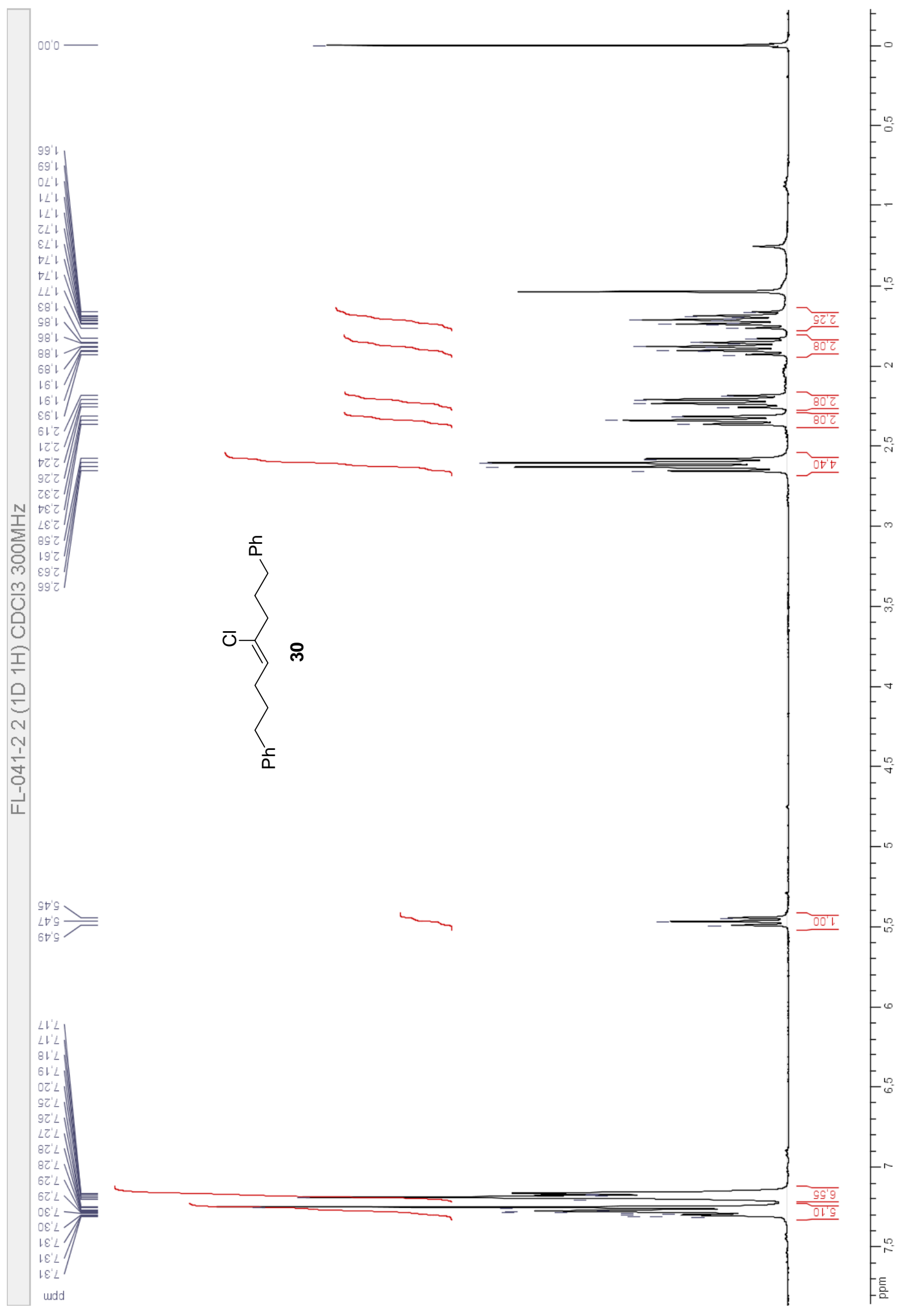

S53 


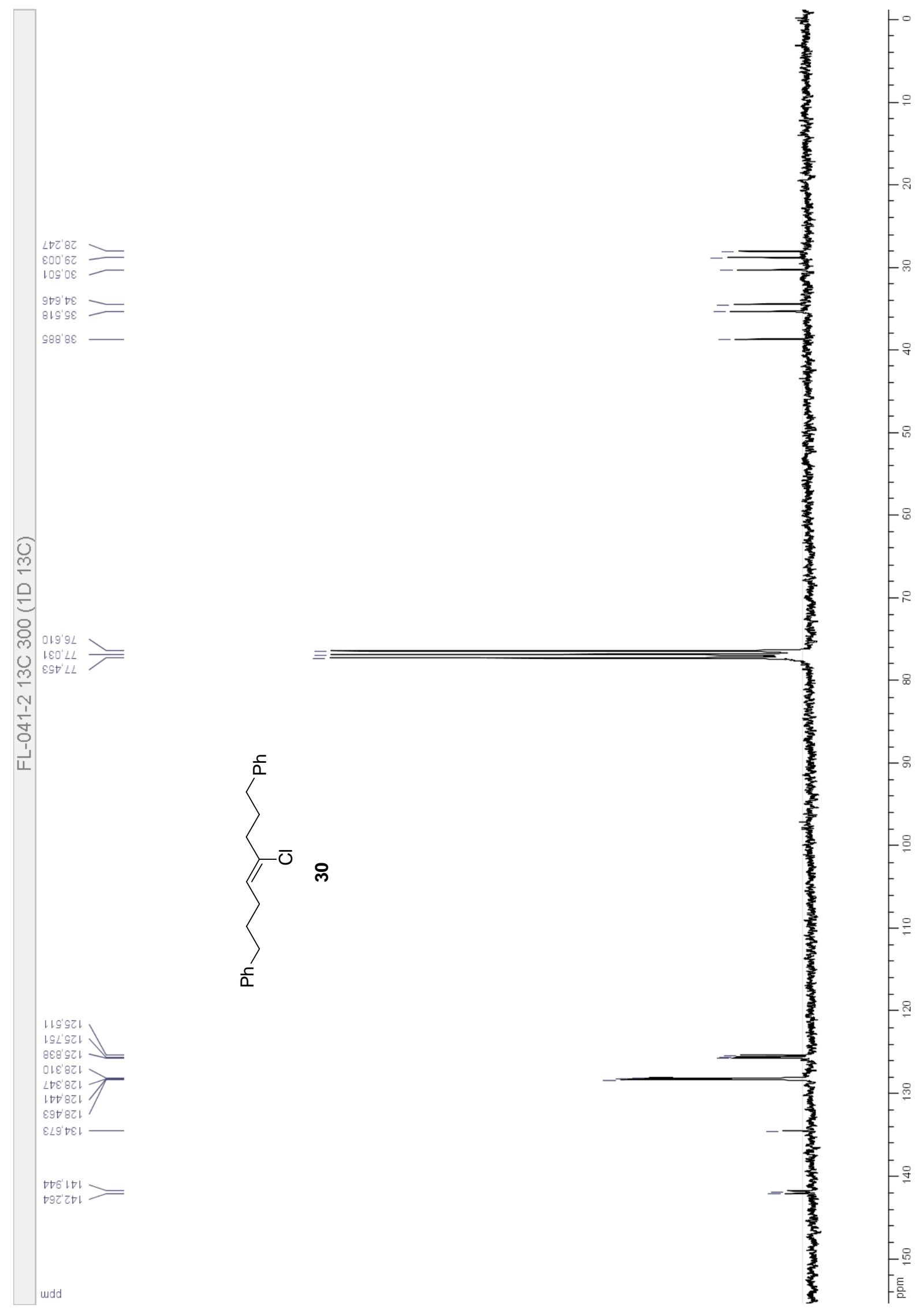




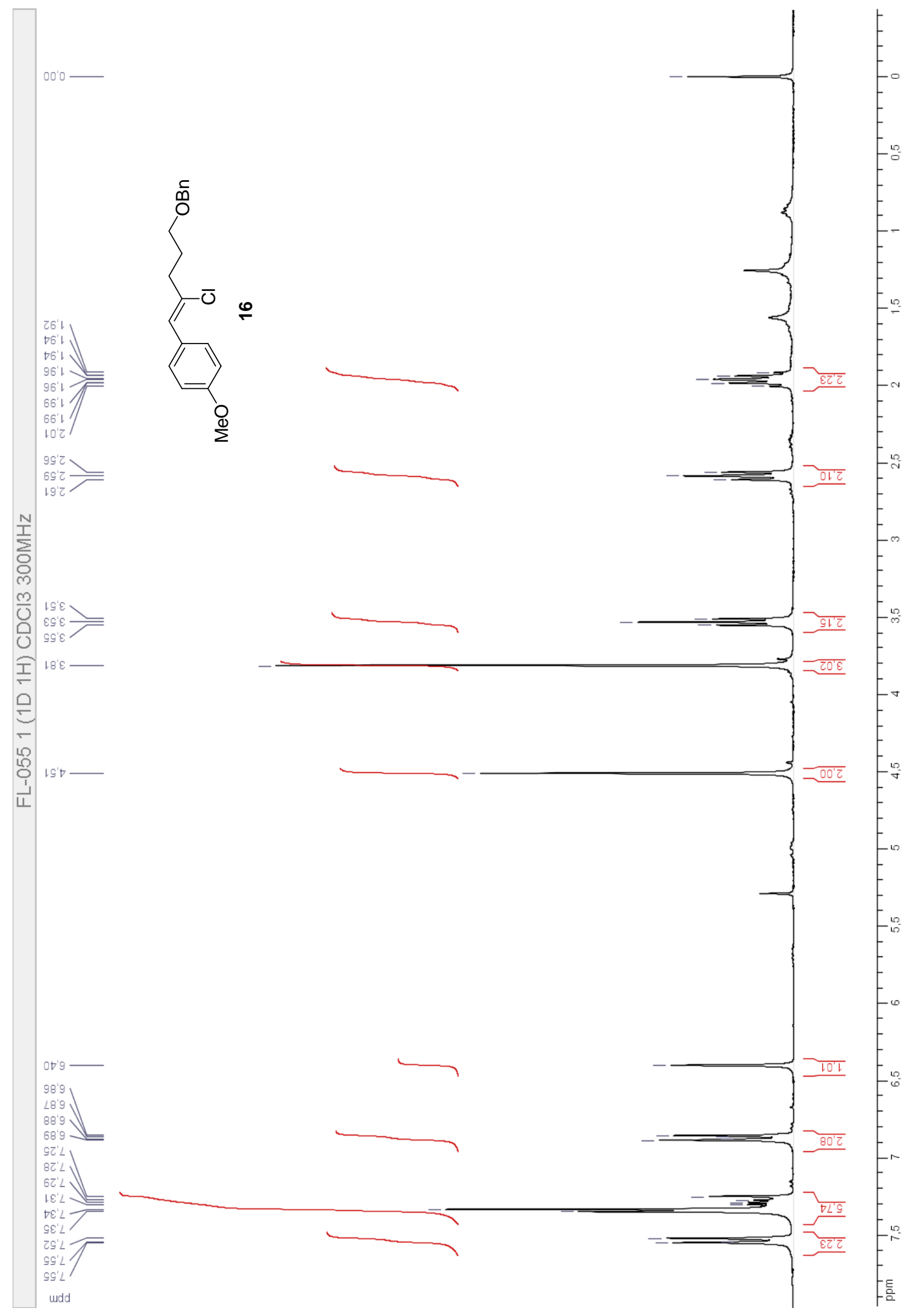




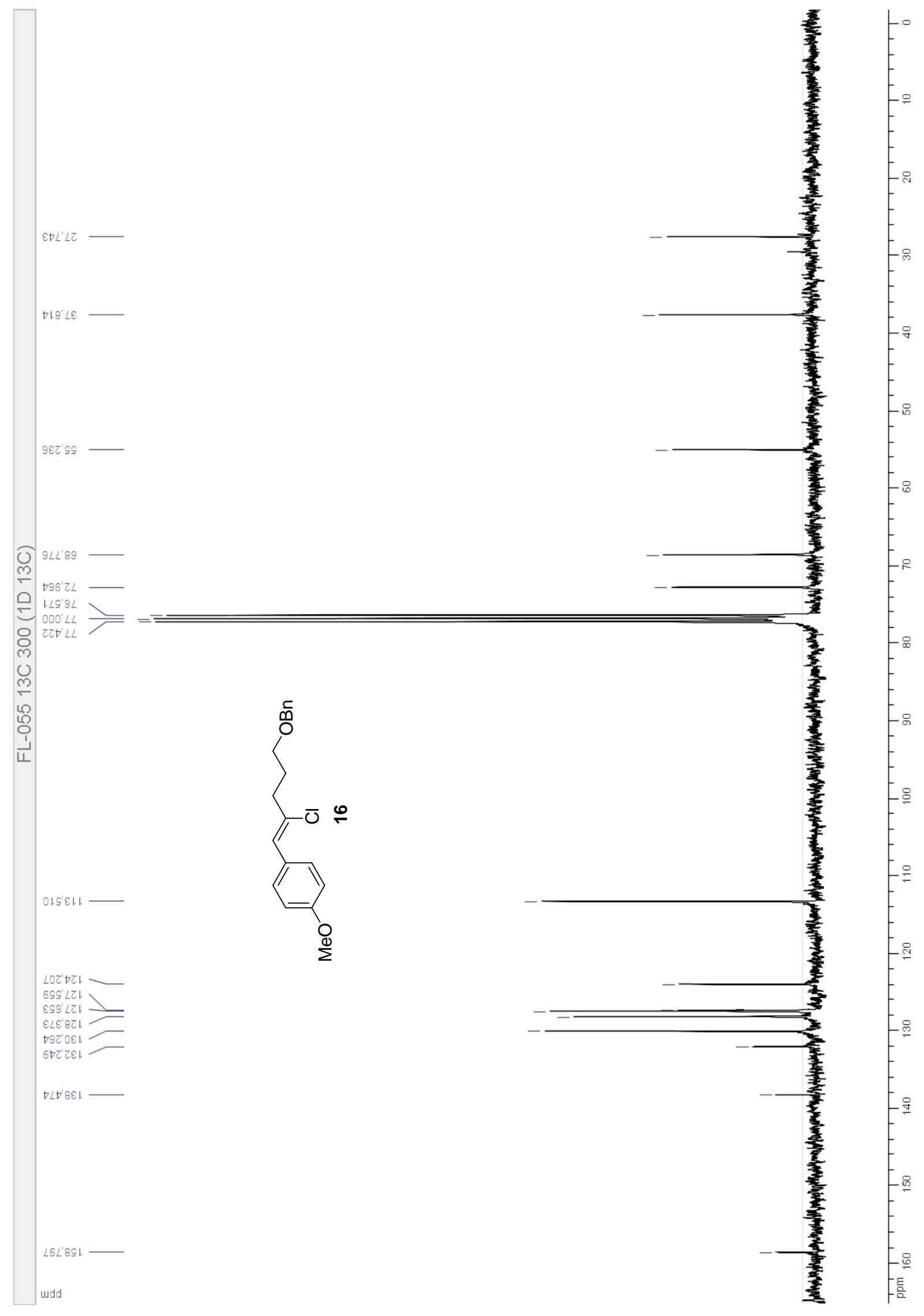




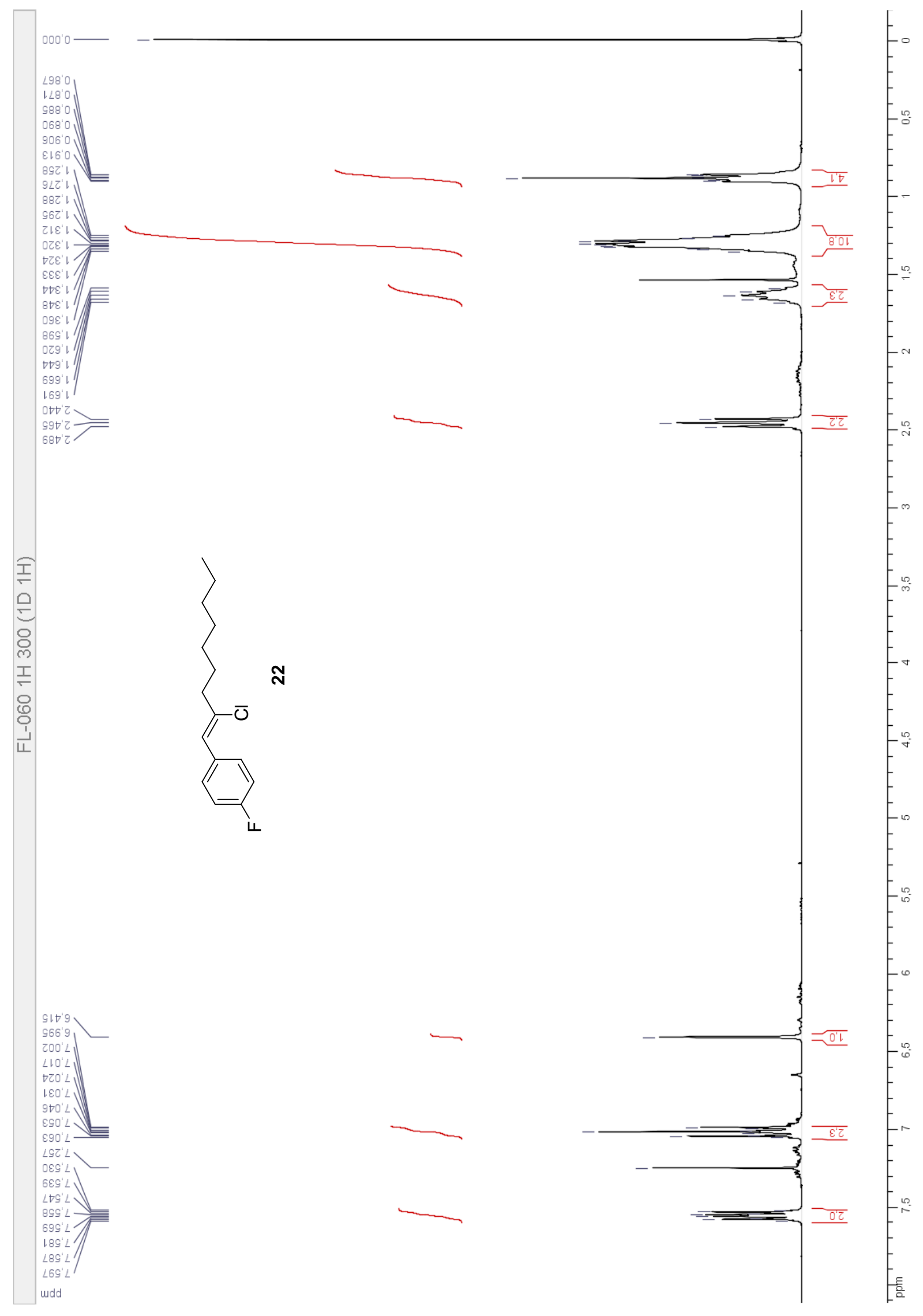



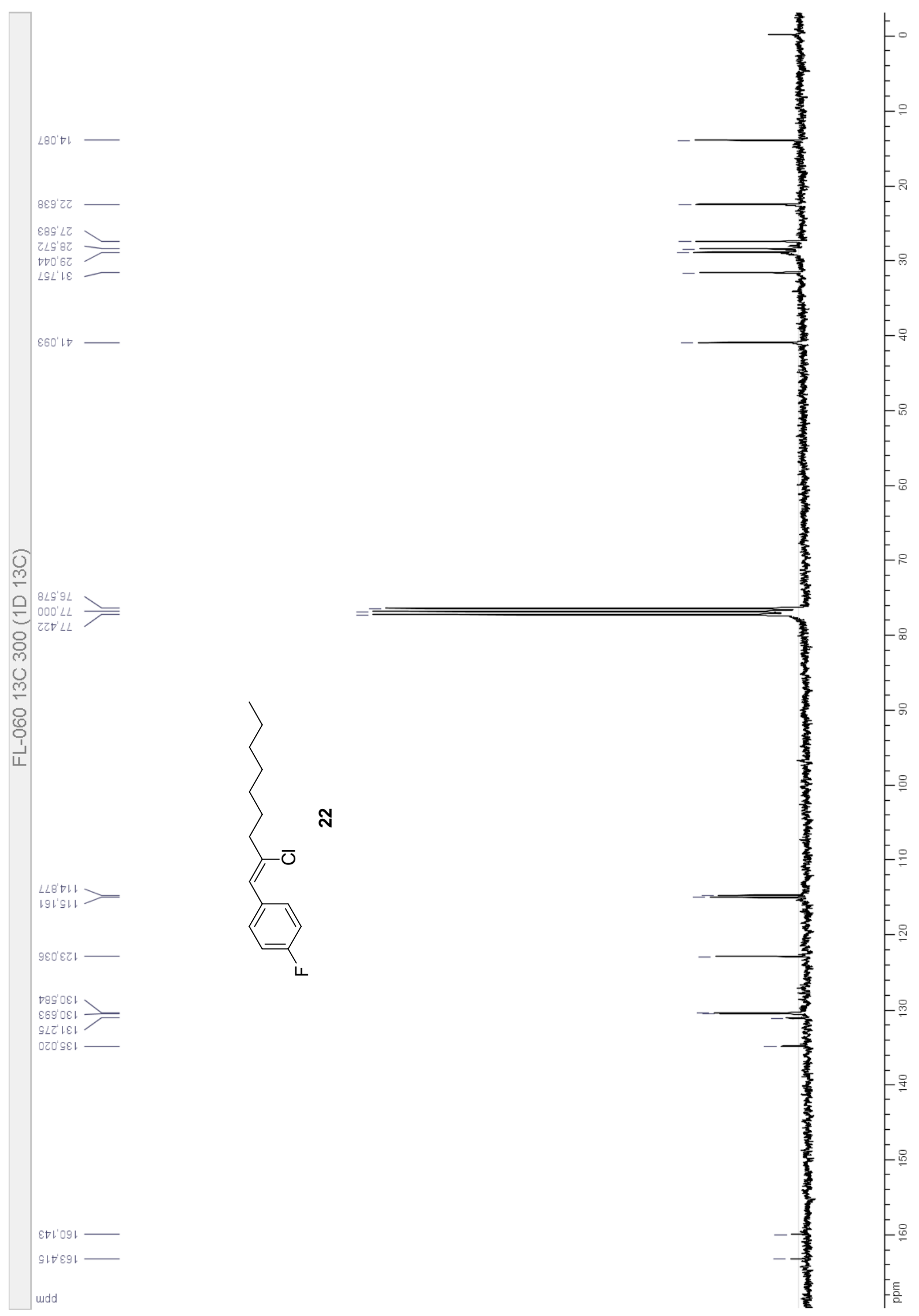

S58 


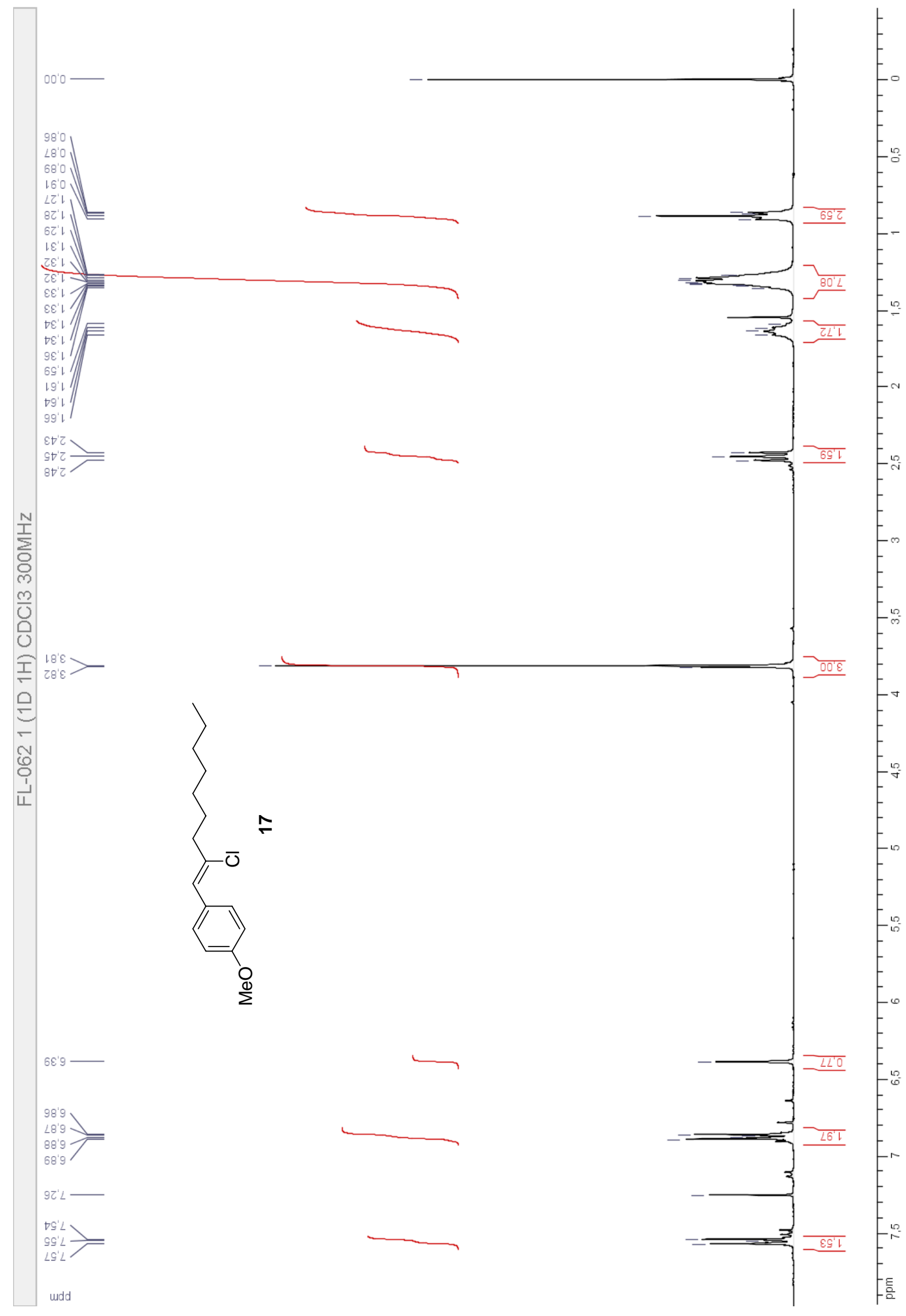




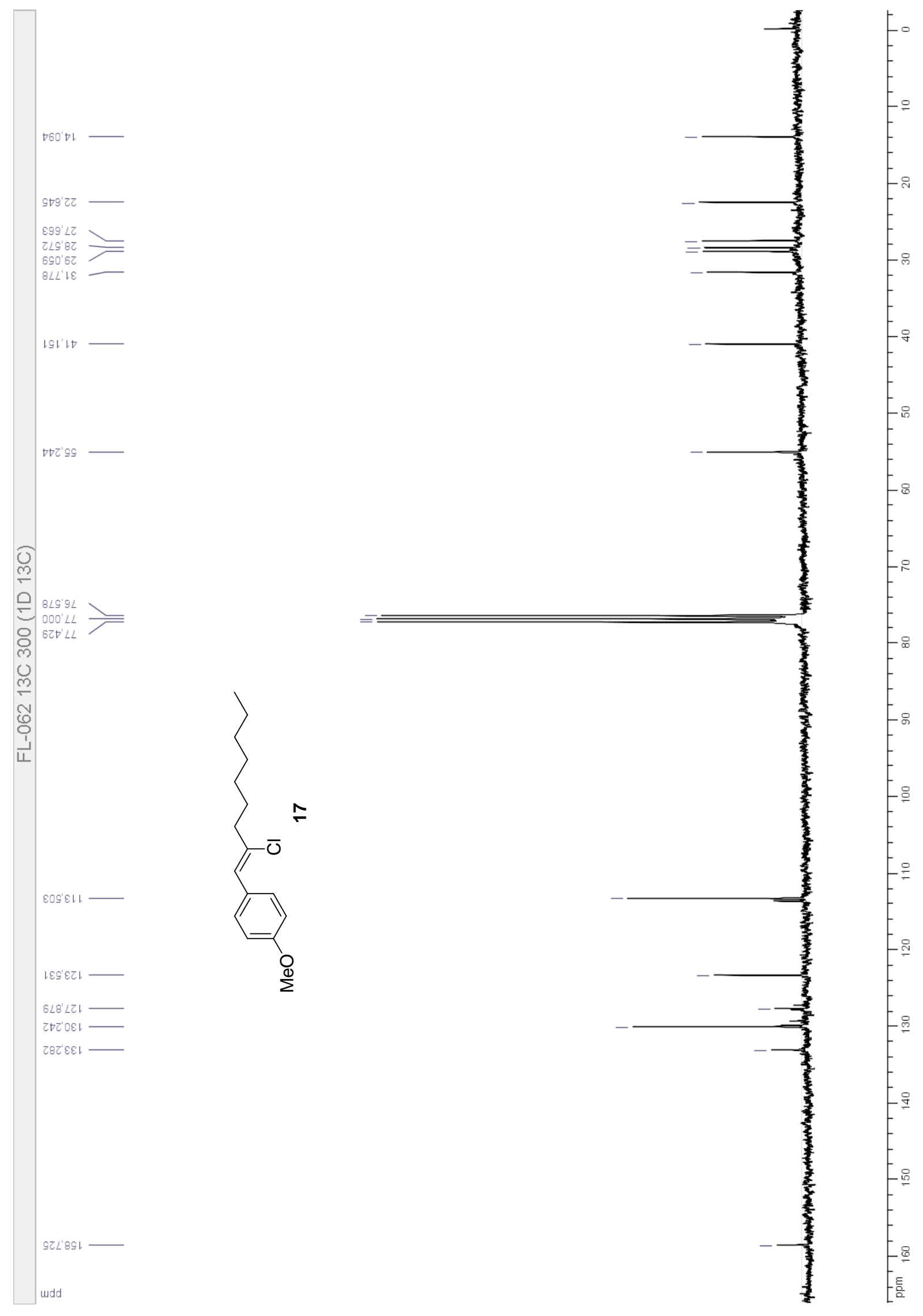




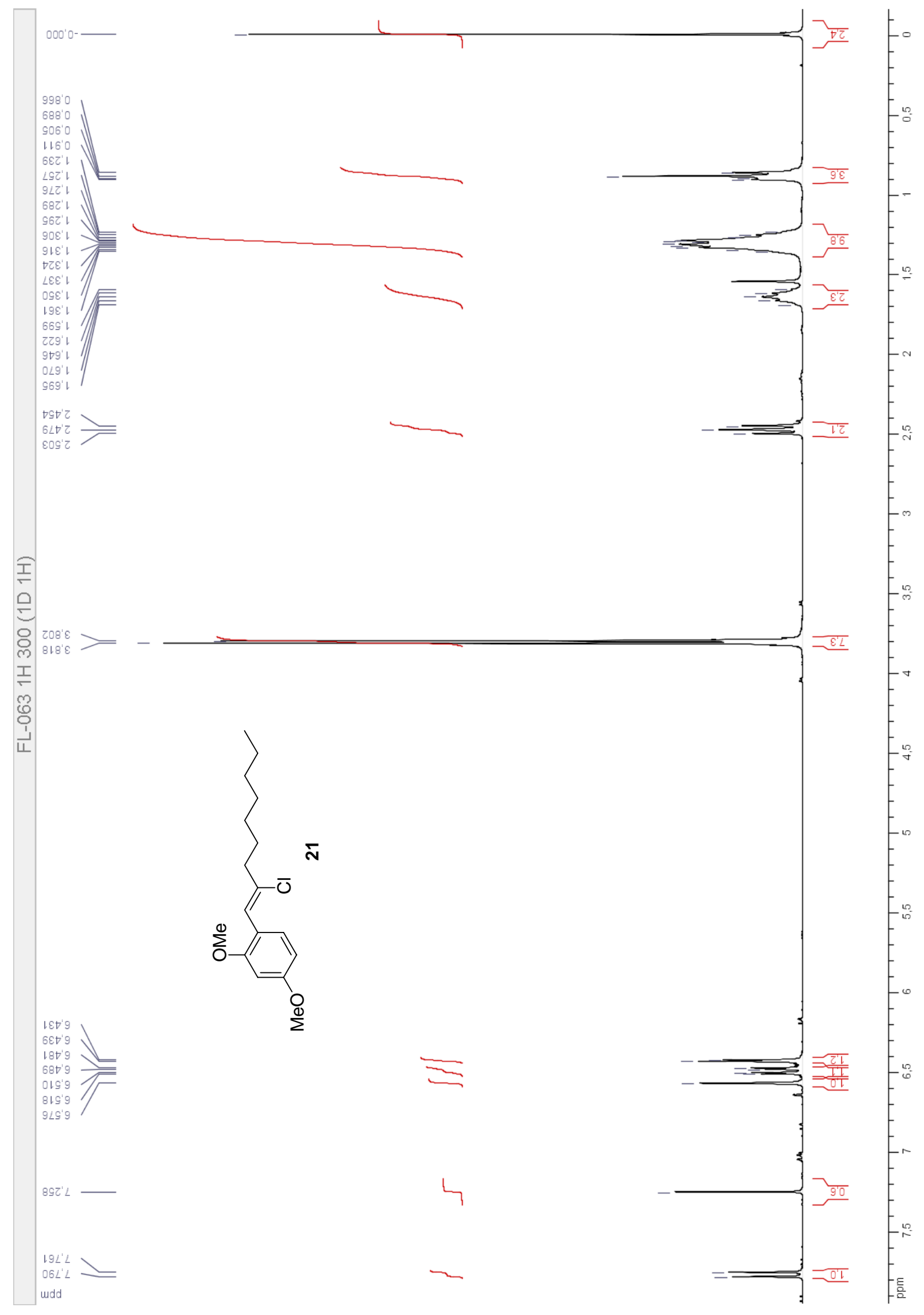




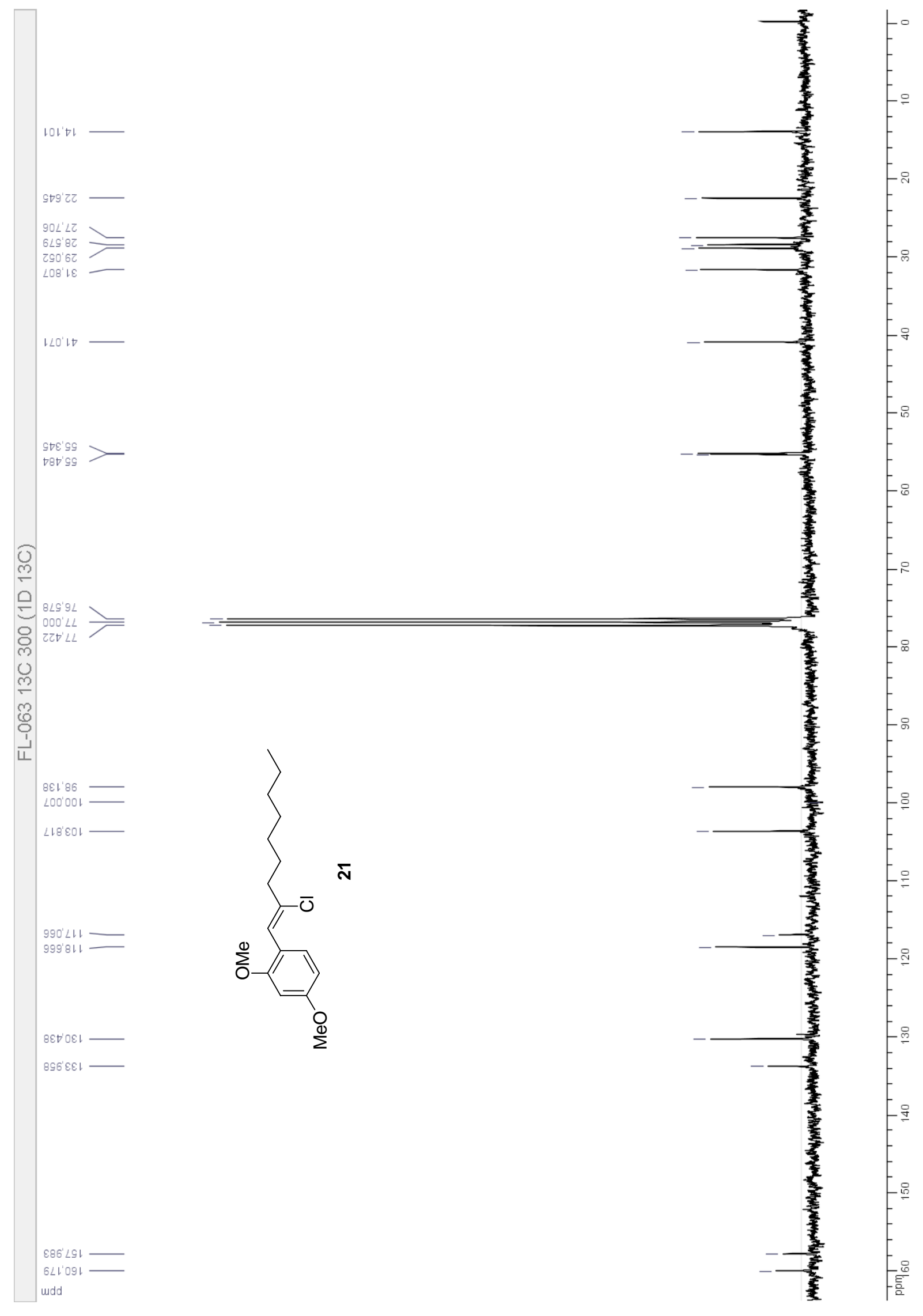




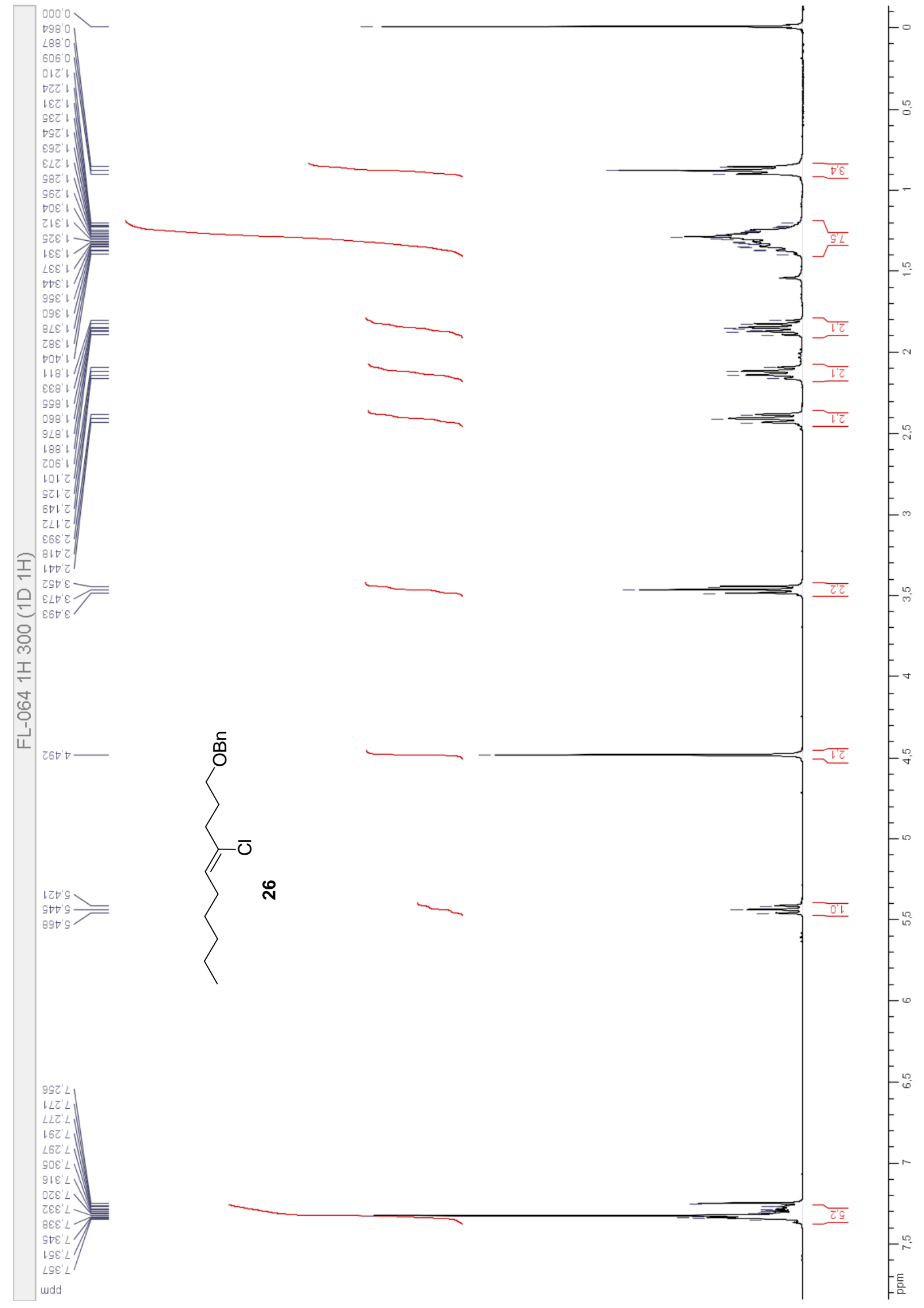



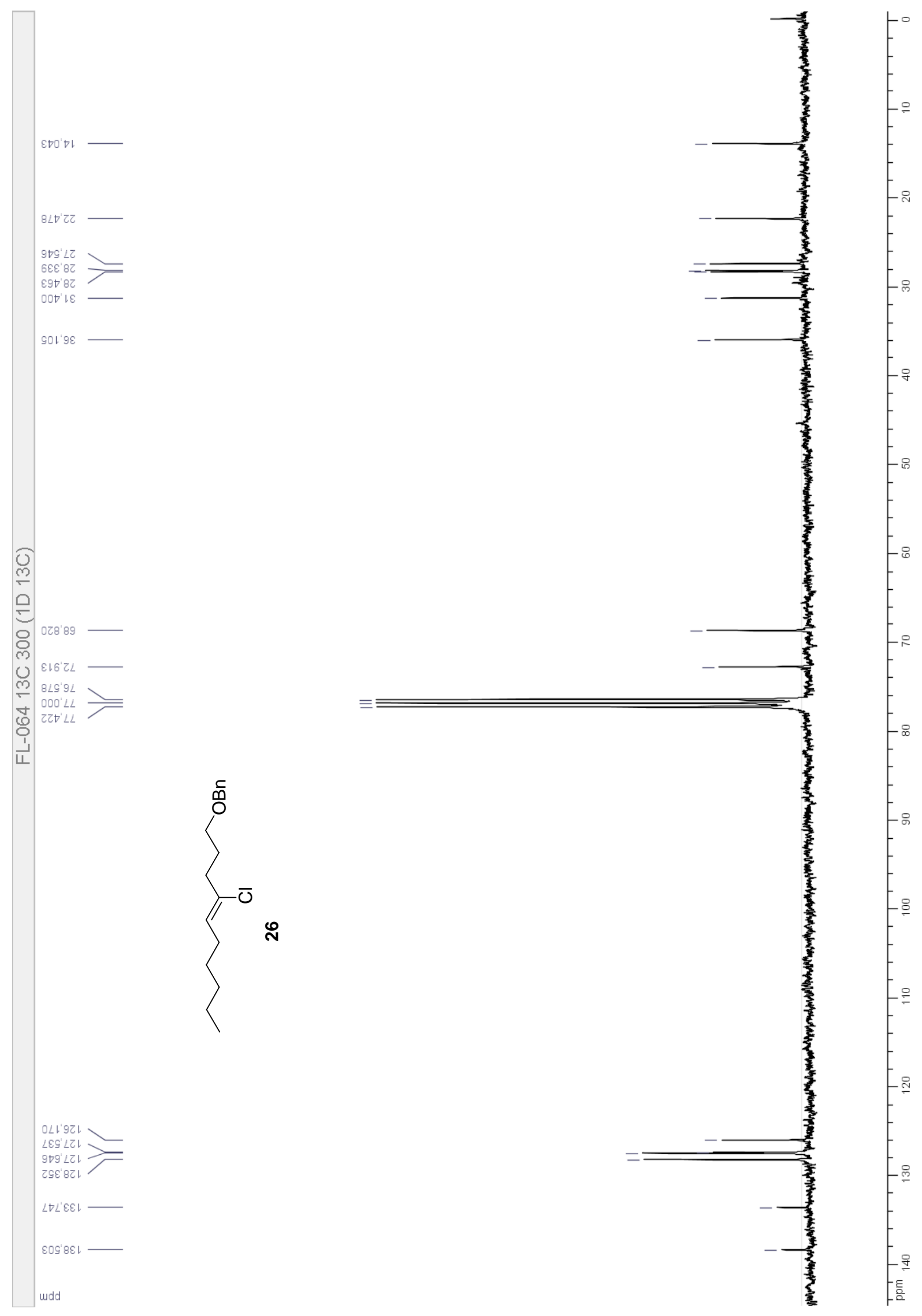

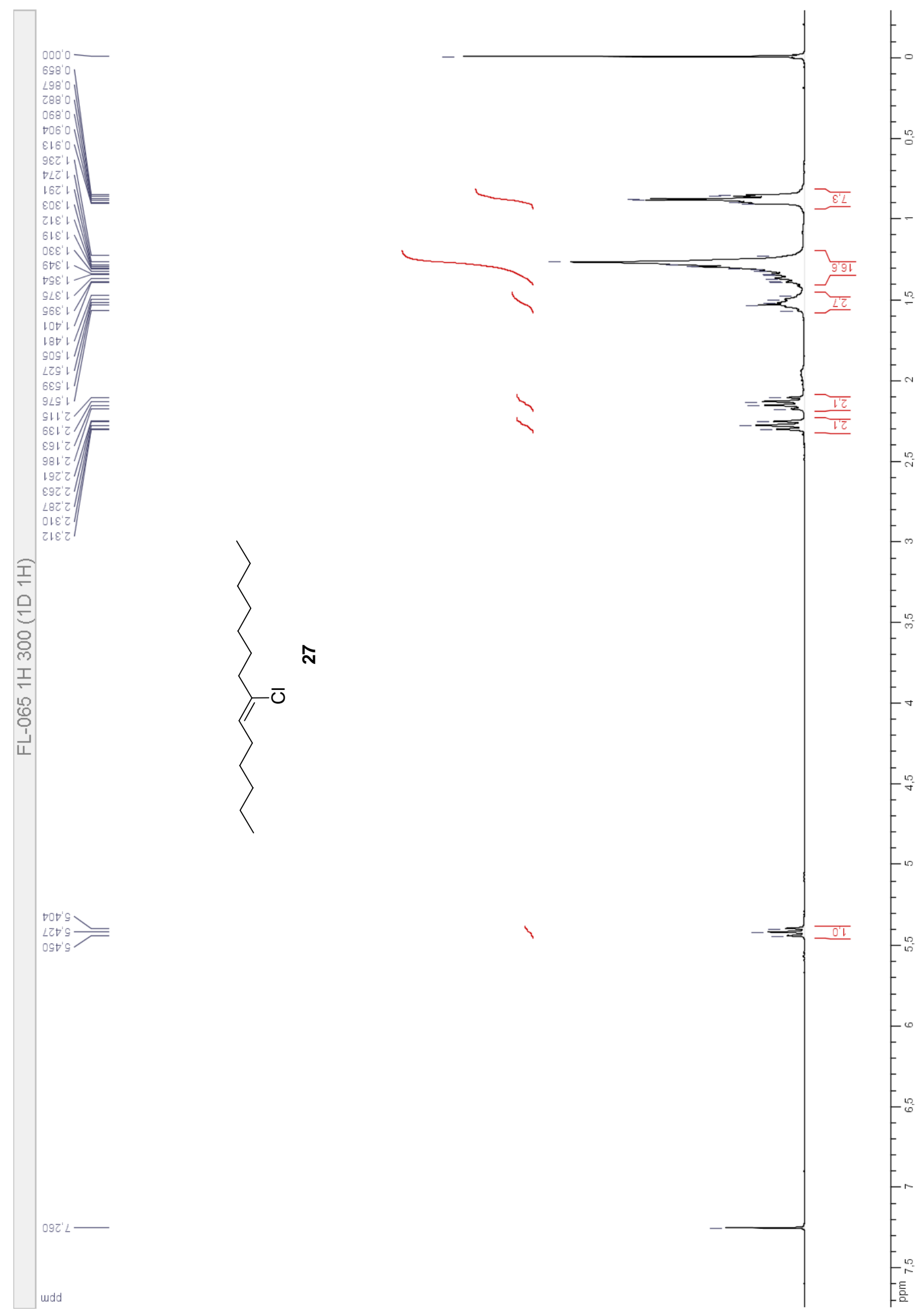


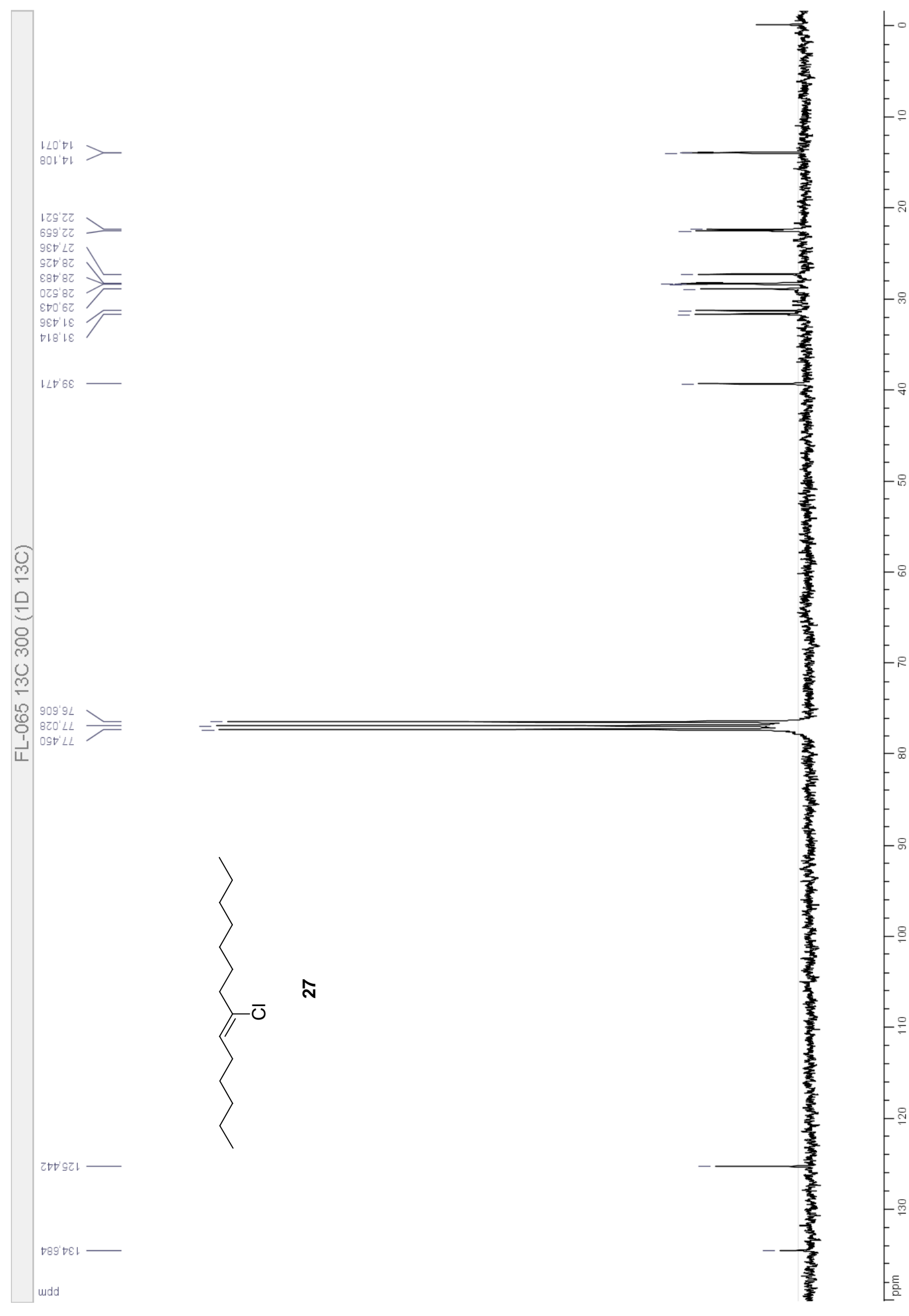




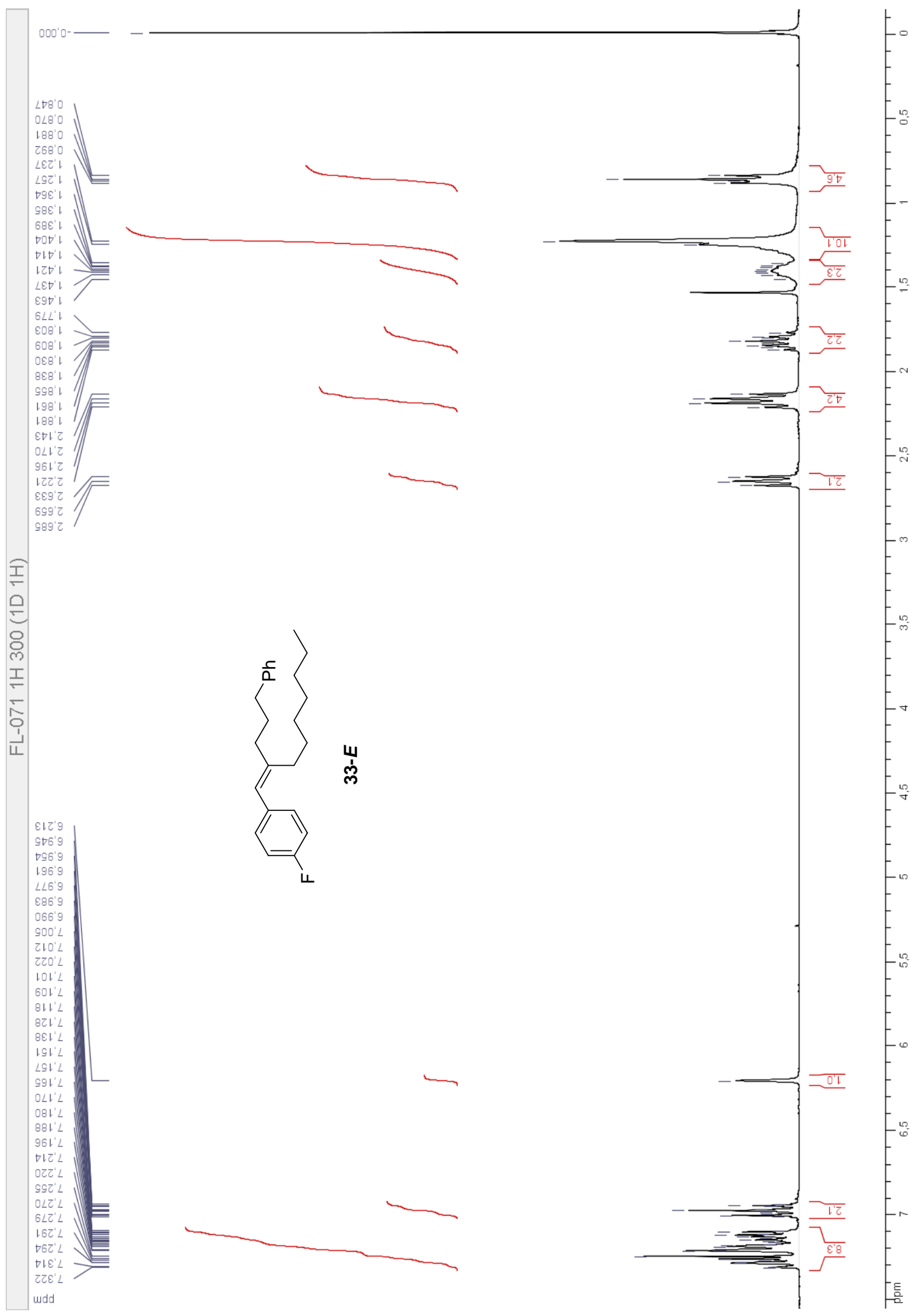




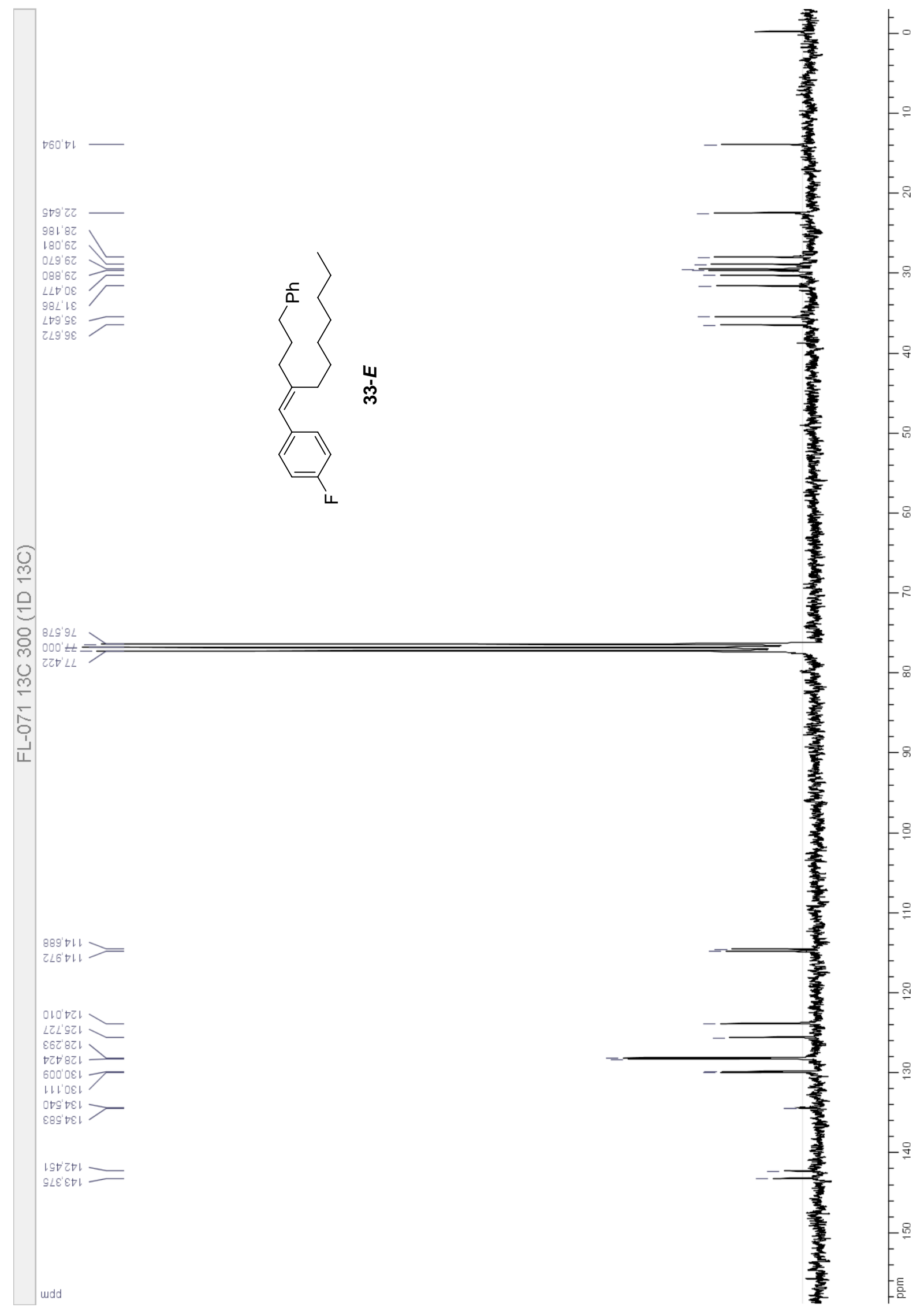




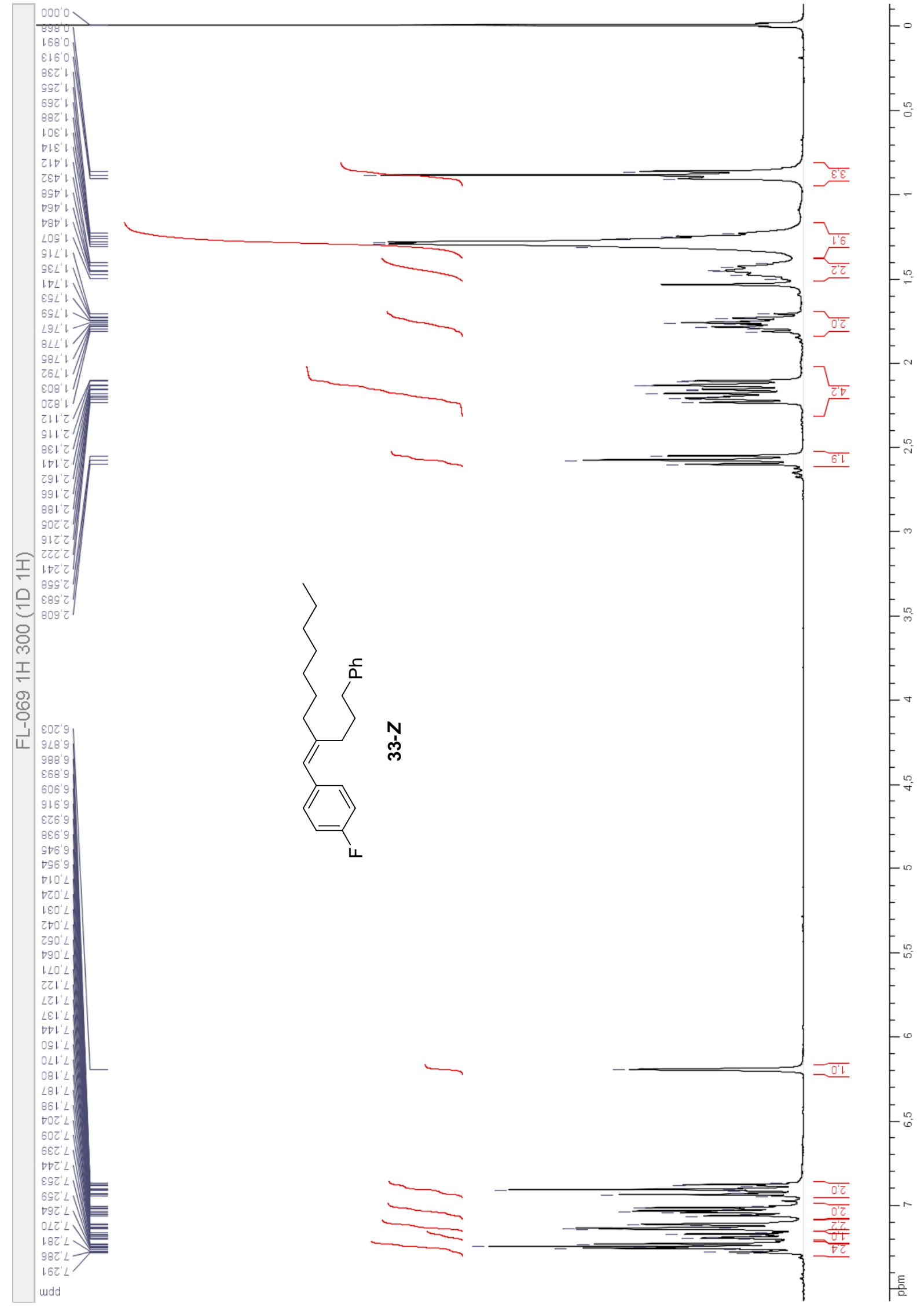




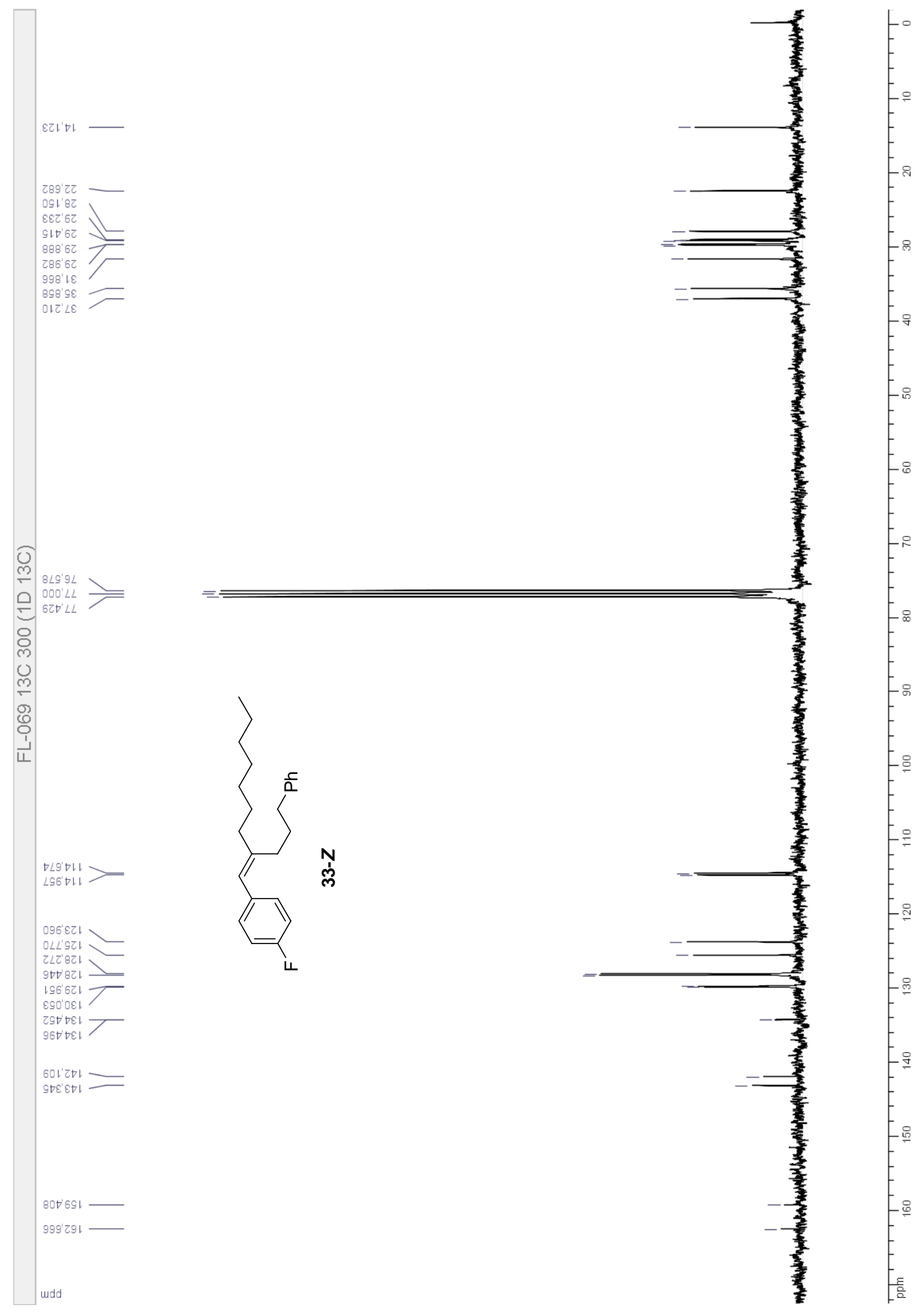




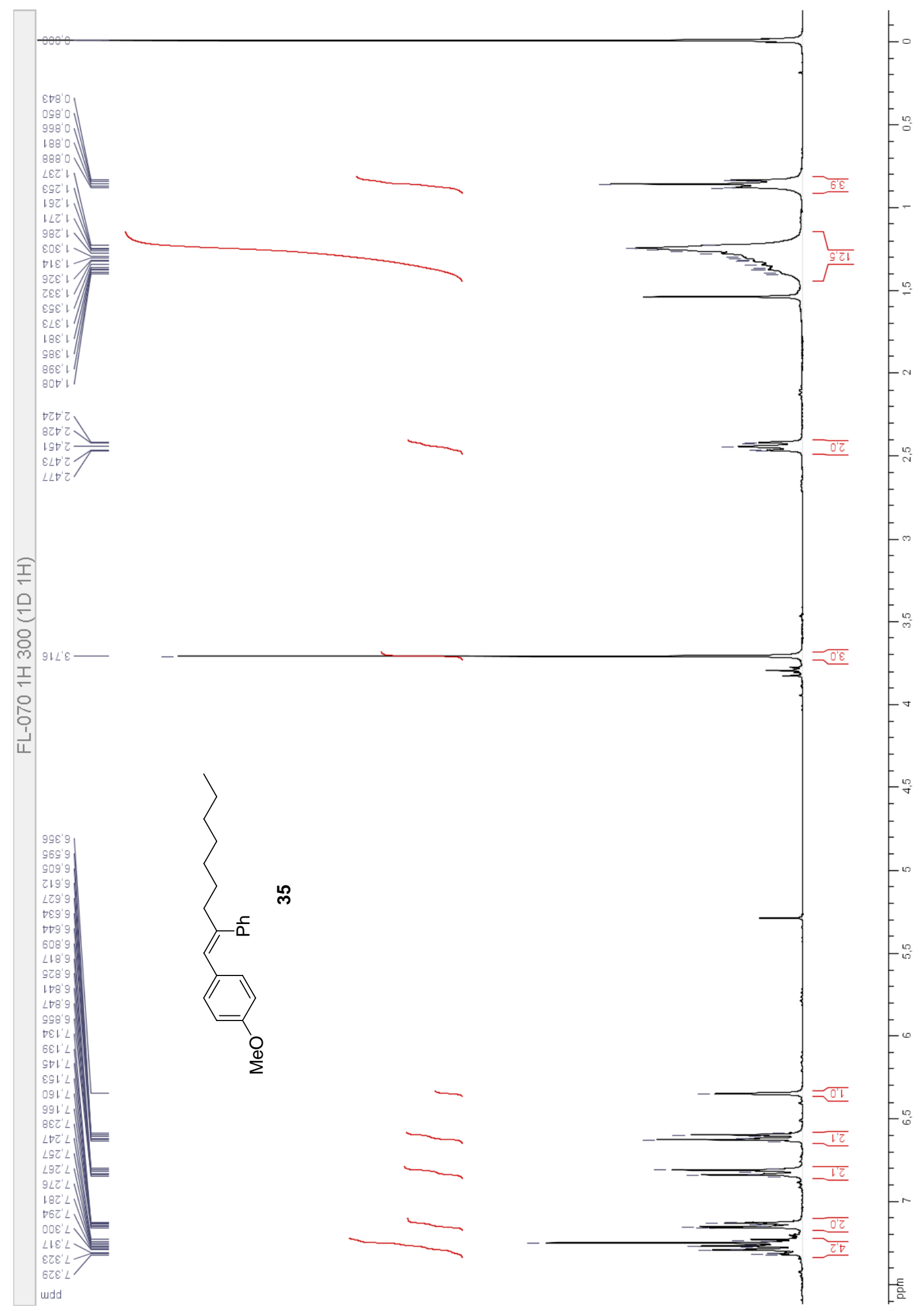




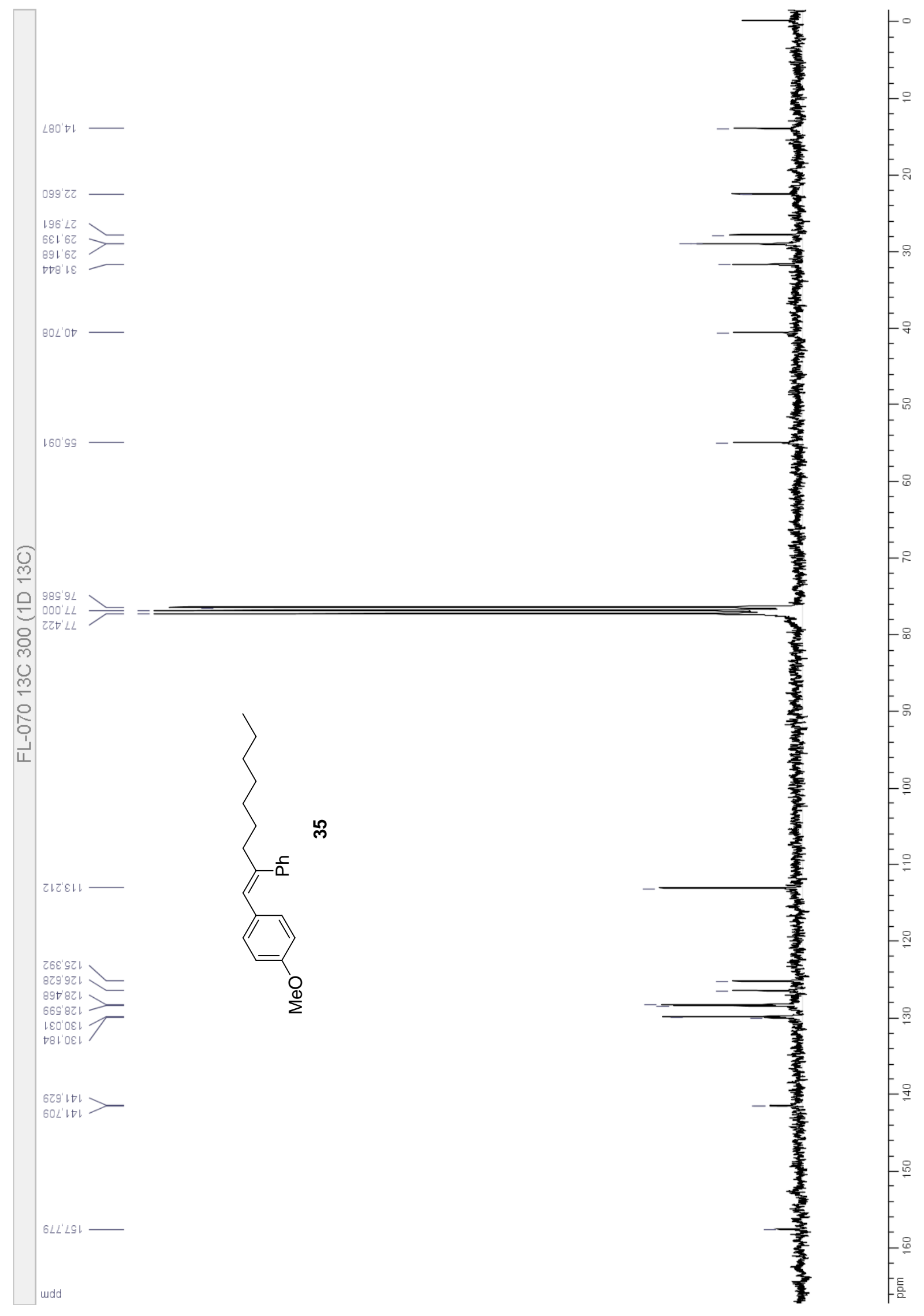




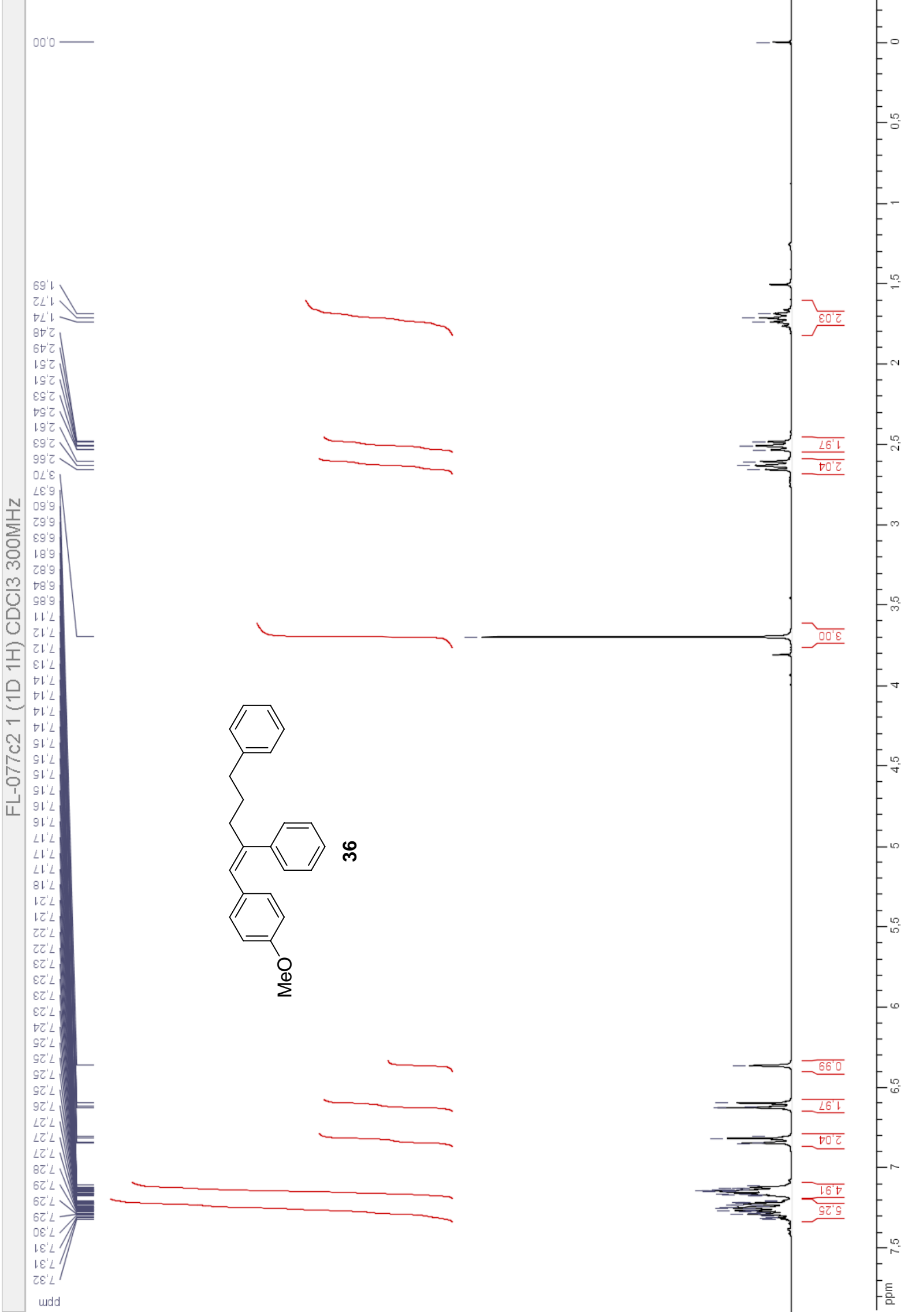




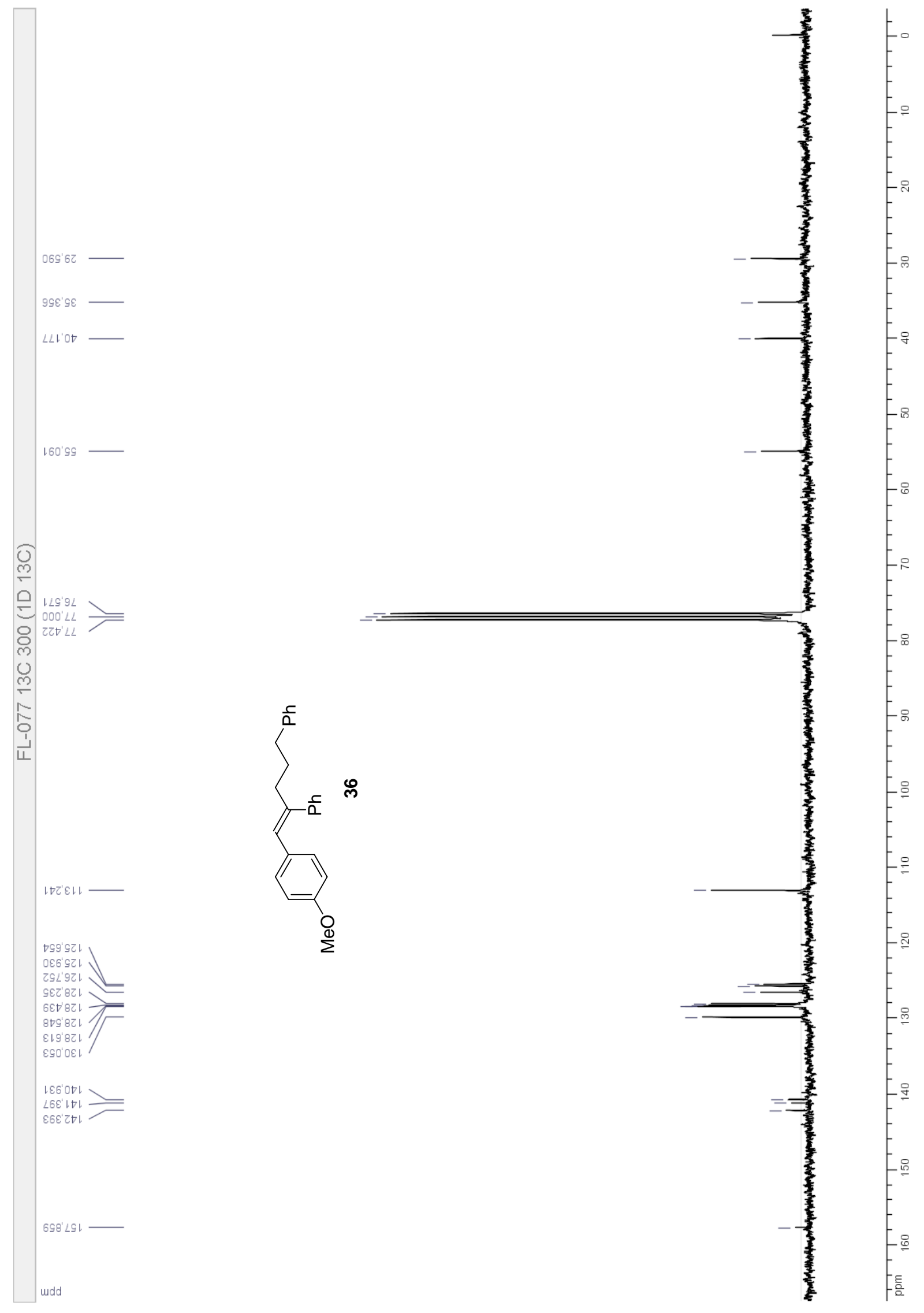




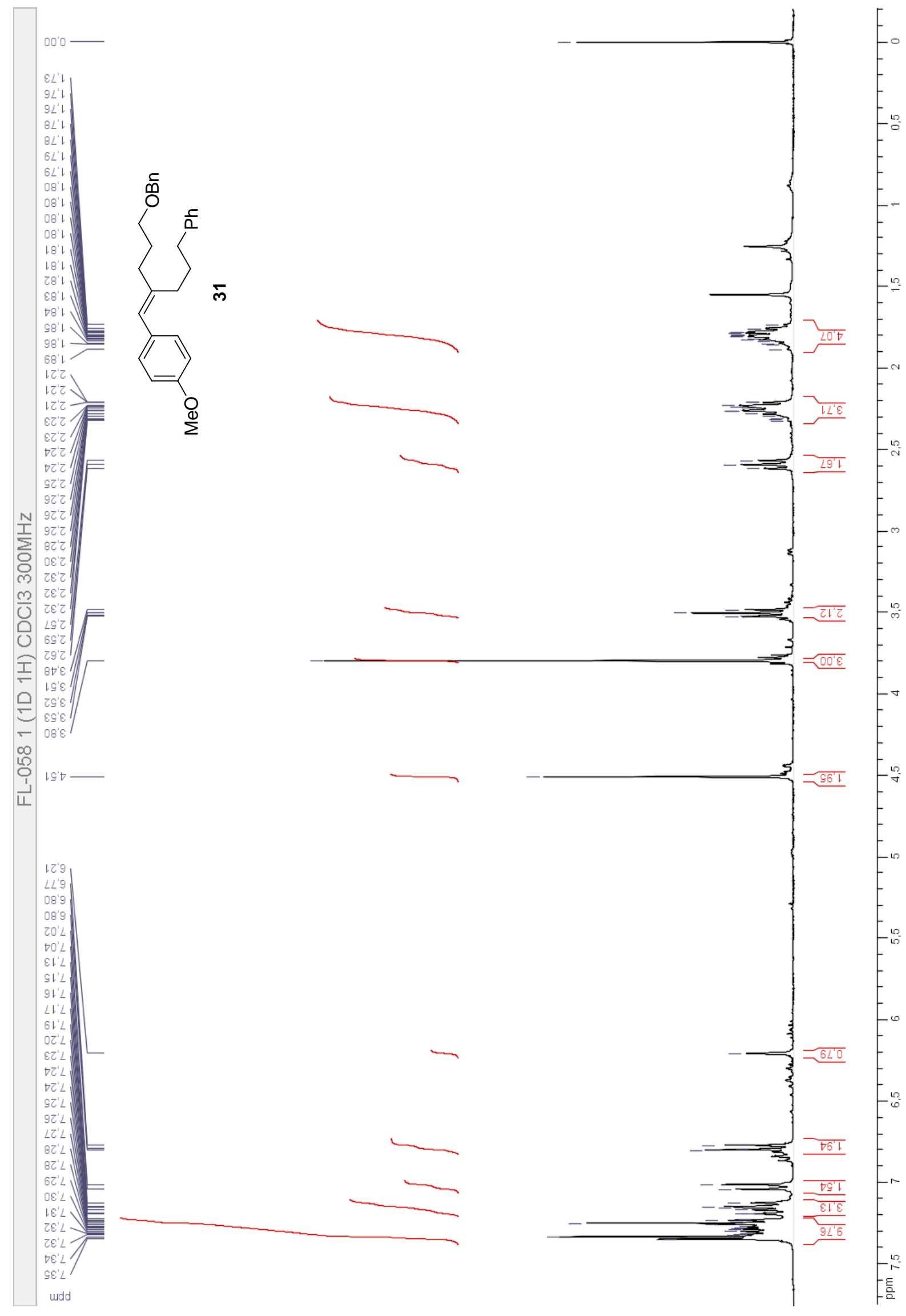




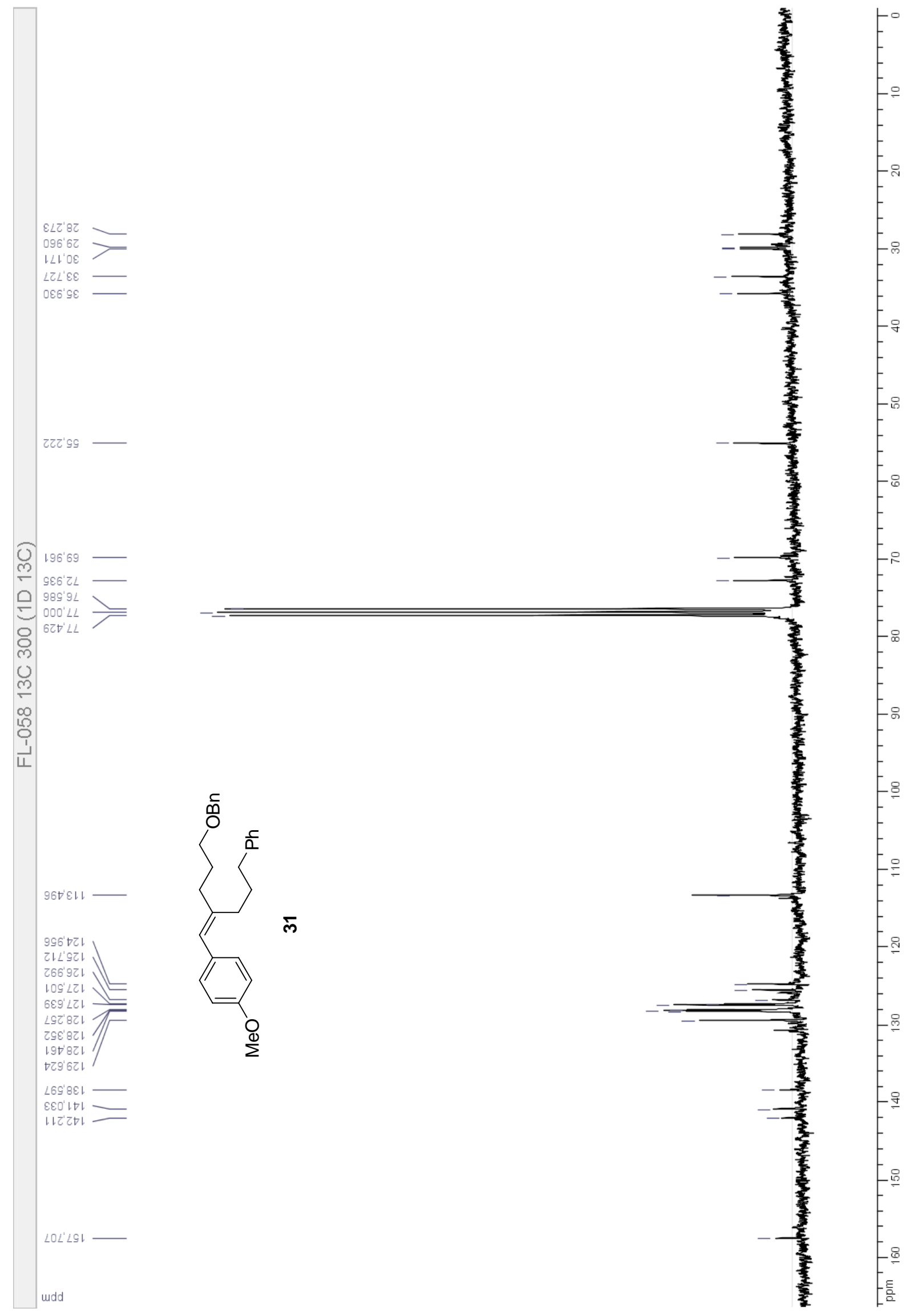




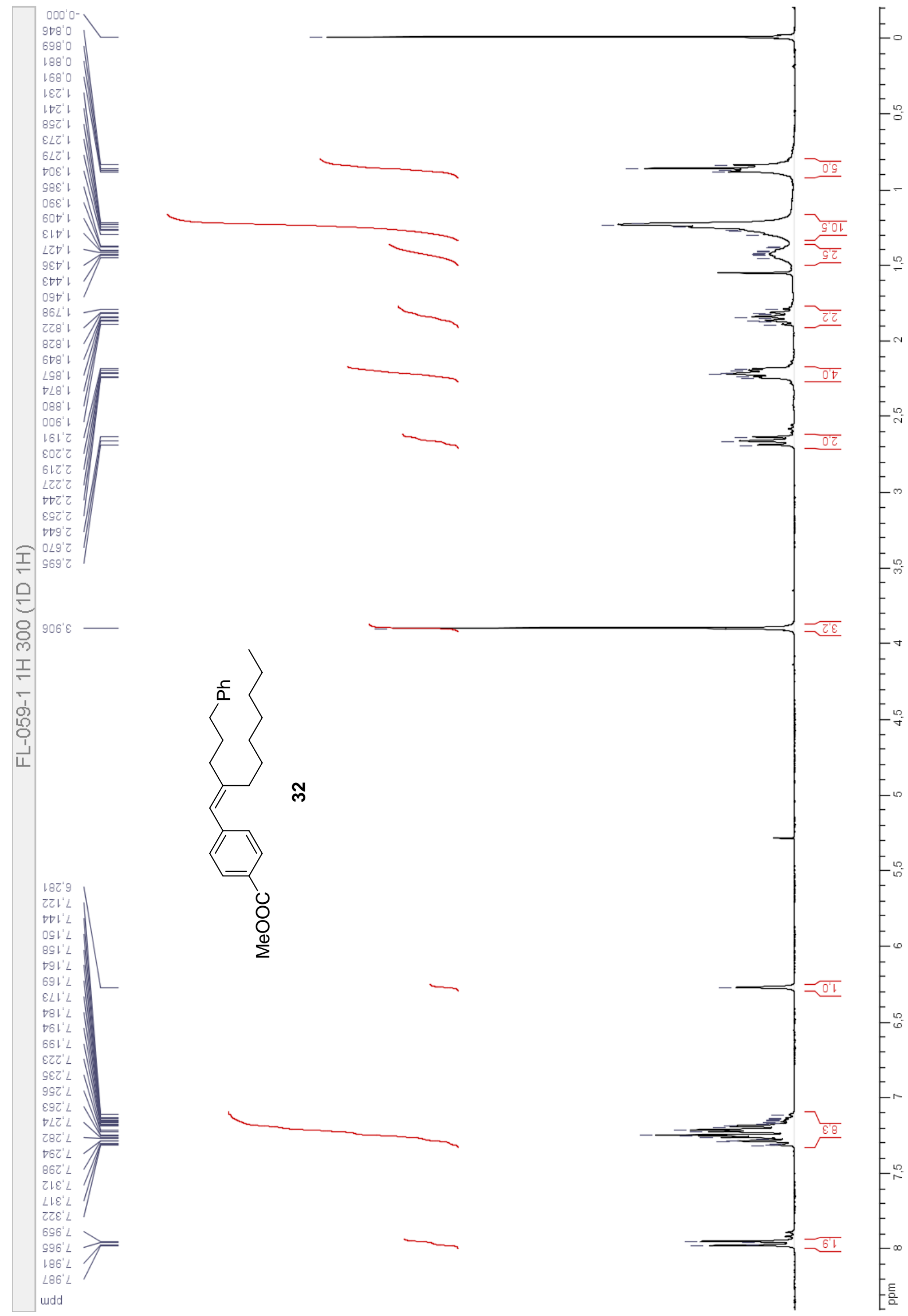




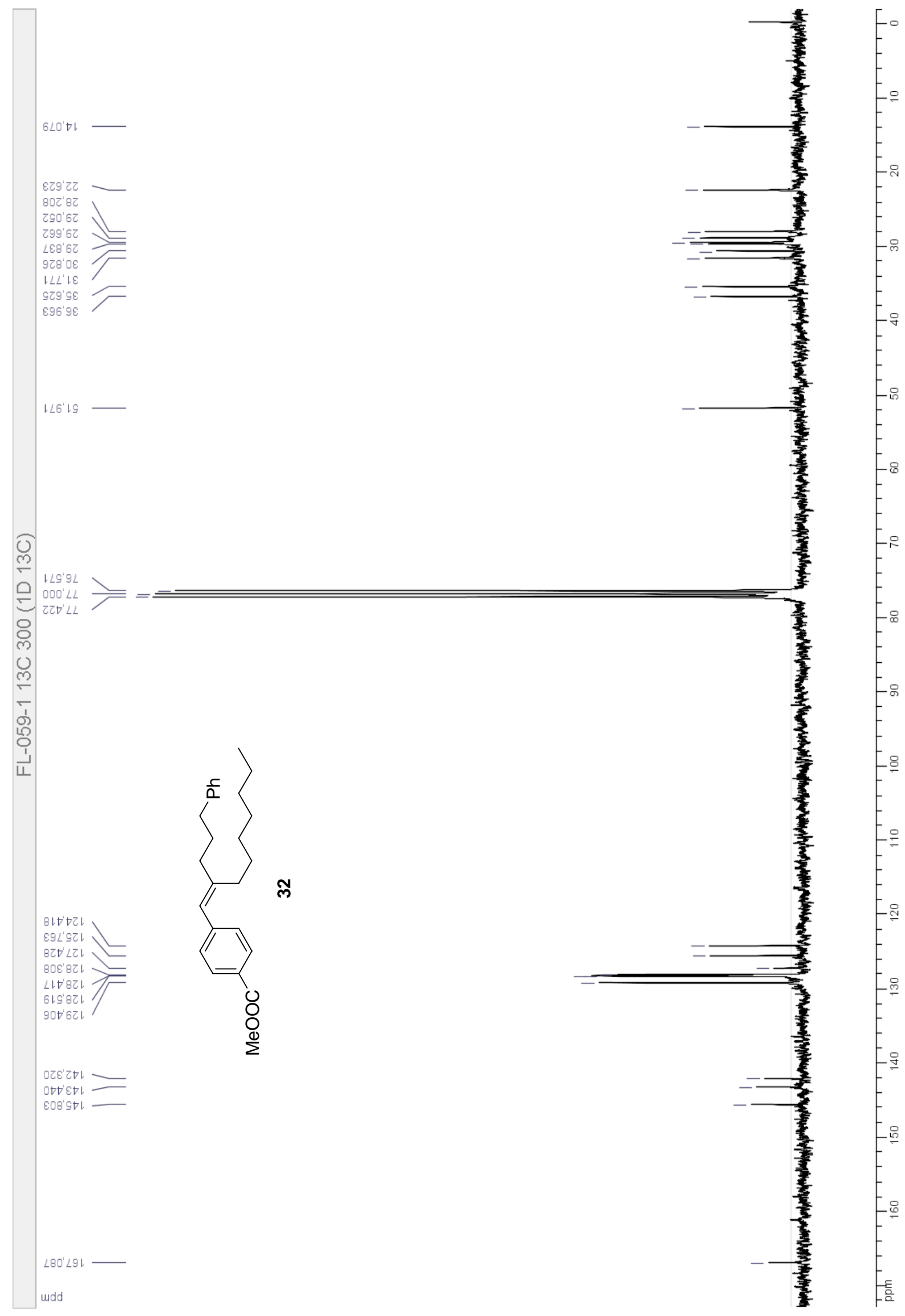

